\title{
Alkylidene Dihydropyridines are Surrogates for Pyridylic Anions in the Conjugate Addition to $\alpha, \beta$-Unsaturated Ketones
}

\author{
Jiaqi Shi, ${ }^{\dagger}$ Ashik Sayyad, ${ }^{\dagger}$ Dan Fishlock, ${ }^{\ddagger}$ and Arturo Orellana*† \\ † Department of Chemistry, York University. 4700 Keele Street, Toronto ON, Canada M3J 1P3 \\ ‡ Process Chemistry and Catalysis, Synthetic Molecule Technical Development. F. Hoffmann-La \\ Roche Ltd. 4070 Basel Switzerland. \\ aorellan@yorku.ca
}

\section{Contents}

I. General experimental 2

II. Optimization screen of Lewis acids, solvents and 3 temperature for conjugate addition of ADHP

III. Preparation of substrates 4-14

IV. Conjugate addition of alkylidene dihydropyridines to $\alpha, \beta-\quad 15-45$ unsaturated ketones

V. $\quad{ }^{1} \mathrm{H}-,{ }^{13} \mathrm{C}-$ and 2D NMR spectra 46-93 


\section{General experimental:}

All reactions were conducted in flame-dried glassware under an atmosphere of argon using anhydrous solvents unless otherwise specified. Triethylamine $\left(\mathrm{Et}_{3} \mathrm{~N}\right)$ and diisopropylamine (i-Pr ${ }_{2} \mathrm{NH}$ ) were distilled from $\mathrm{CaH}_{2}$ prior to use. Tetrahydrofuran (THF) and acetonitrile $\left(\mathrm{CH}_{3} \mathrm{CN}\right)$ were dispensed from an Inert solvent purification system (model number: PureSolve MD 5). Commercial reagents were used as received. Thin-layer chromatography (TLC) was performed on SiliCycle silica gel 60 F254 plates. Visualization was carried out using UV light (254 nm), $\mathrm{KMnO}_{4}$, and 2,4-dinitrophenylhydrazine (DNP) solutions. Hexanes, diethyl ether, pentane and ethyl acetate (all ACS grade) were used as received. Flash column chromatography was carried out using SiliCycle silica gel (230-400 mesh, 40-63 $\mu \mathrm{m}, 60 \AA$ pore size). ${ }^{1} \mathrm{H}-\mathrm{NMR}$ and ${ }^{13} \mathrm{C}-\mathrm{NMR}$ were recorded on a Bruker $400 \mathrm{AV}$ or Bruker $300 \mathrm{AV}$ spectrometer in chloroform-D (99.8\% deuterated). Spectra recorded using chloroform-D were calibrated to $7.26 \mathrm{ppm}$ for ${ }^{1} \mathrm{H}$ and 77.16 ppm for ${ }^{13} \mathrm{C}$. Chemical shifts $(\delta)$ are reported in ppm and multiplicities are indicated by s (singlet), $d$ (doublet), dd (doublet of doublet), ddd (doublet of doublet of doublet), $t$ (triplet), $q$ (quartet), $p$ (quintet), m (multiplet), br (broad). Coupling constants $(J)$ are reported in $\mathrm{Hz}$ (Hertz).

\section{General procedure (A). Synthesis of alkylidene dihydropyridines (ADHPs):}

A dry round-bottomed flask was charged with a $0.1 \mathrm{M}$ solution of 4-alkylpyridine (1.0 equiv.) in anhydrous THF, and $\mathrm{Et}_{3} \mathrm{~N}$ (3.0 equivs.). The mixture was cooled to $0{ }^{\circ} \mathrm{C}$ while being stirred for 5 minutes. Unless otherwise stated, 2.0 equivs. of ethyl chloroformate were added dropwise to the above reaction mixture at $0{ }^{\circ} \mathrm{C}$ while stirring. The reaction mixture was stirred at room temperature for 30 minutes and then concentrated using a rotary evaporator. The residue was dissolved in diethyl ether, triturated for 2-3 minutes and filtered through a cotton plug to remove the precipitated triethylammonium chloride salt. The filtrate was concentrated using a rotary evaporator and the corresponding ADHP obtained was used for next step without further purification.

\section{General Procedure (B). Conjugate addition of alkylidene dihydropyridines to $\alpha, \beta$ - unsaturated ketones:}

A dry round-bottomed flask was charged with a $0.2 \mathrm{M}$ solution of the $\alpha, \beta$-unsaturated ketone (1.0 equiv.) in anhydrous $\mathrm{CH}_{3} \mathrm{CN}$. The solution was cooled to $-40{ }^{\circ} \mathrm{C}, 1.1$ equivs. of triethylsilyl triflate (TESOTf) were added dropwise, and the reaction mixture was stirred for 15 minutes. Unless otherwise stated, the ADHP was dissolved in anhydrous $\mathrm{CH}_{3} \mathrm{CN}(0.2 \mathrm{M})$ and added dropwise to the above reaction mixture while maintaining the temperature at $-40{ }^{\circ} \mathrm{C}$. The mixture was stirred at $-40{ }^{\circ} \mathrm{C}$, and the reaction progress was monitored by TLC and ${ }^{1} \mathrm{H}-\mathrm{NMR}$. Upon consumption of the ADHP, as shown by ${ }^{1} \mathrm{H}-\mathrm{NMR}$, the reaction mixture was placed in an ice-water bath and quenched with 4.0 equivs. of $1 \mathrm{M}$ aqueous $\mathrm{NaOH}$. The mixture was stirred vigorously until the hydrolysis of the carbamate and the removal of TES group were complete, as indicated by TLC. The mixture was extracted with EtOAc $(4 \times 10 \mathrm{~mL})$. The combined organic layers were washed with brine, dried with $\mathrm{MgSO}_{4}$, filtered through a cotton plug, and concentrated using a rotary evaporator. The crude reaction mixture was purified by flash column chromatography to obtain the desired conjugate addition product. 
Optimization screen of Lewis acids, solvents and temperatue for conjugate addition of ADHP:

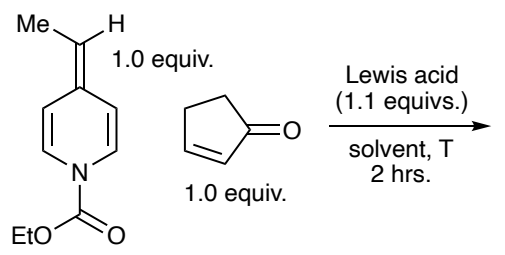<smiles>CC(c1cc[nH+]cc1)C1CCC(=O)C1</smiles>

4-Ethylpyridine $(0.12 \mathrm{~mL}, 1.0$ equiv., $1.0 \mathrm{mmol})$ was converted to the corresponding ADHP following general procedure $A$.

A dry round-bottomed flask was charged with a $0.2 \mathrm{M}$ solution of 2-cyclopenten-1-one(1.0 equiv.) in anhydrous solvent as listed in Table 1. The solution was cooled to the temperature as listed in the Table 1.1.1 equivs. of Lewis acid were added to the ketone solution, and the reaction mixture was stirred for 15 minutes. The ADHP was dissolved in anhydrous solvent $(0.2 \mathrm{M})$ and added dropwise to the above reaction mixture while maintaining the same temperature. The mixture was stirred at the same temperature or slowly warmed up to certain temperature as listed in the table. Upon consumption of the ADHP, as shown by ${ }^{1} \mathrm{H}-\mathrm{NMR}$, the reaction mixture was placed in an ice-water bath and quenched with 4.0 equivs. of $1 \mathrm{M}$ aqueous $\mathrm{NaOH}$. The mixture was stirred vigorously for 1 hour. The mixture was extracted with EtOAc $(4 \times 10 \mathrm{~mL})$. The combined organic layers were washed with brine, dried with $\mathrm{MgSO}_{4}$, filtered through a cotton plug, and concentrated using a rotary evaporator. The crude reaction mixture was purified by flash column chromatography to obtain the desired conjugate addition product 3B.

Table 1: Optimization screen of Lewis acids, solvents and temperature

\begin{tabular}{|c|c|c|c|c|}
\hline Entry & Acid & $\mathbf{T}$ & Solvent & Yield \\
\hline 1 & TESOTf & -40 to $0{ }^{\circ} \mathrm{C}$ & $\mathrm{CH}_{3} \mathrm{CN}$ & $50 \%$ \\
\hline 2 & TESOTf & $-40^{\circ} \mathrm{C}$ to r.t. & $\mathrm{CH}_{3} \mathrm{CN}$ & $38 \%$ \\
\hline 3 & TESOTf & $-40^{\circ} \mathrm{C}$ & THF & $28 \%$ \\
\hline 4 & TESOTf & $-40^{\circ} \mathrm{C}$ & DCM & $66 \%$ \\
\hline 5 & $\mathrm{BF}_{3} \cdot \mathrm{OEt}_{2}$ & $-40^{\circ} \mathrm{C}$ & $\mathrm{CH}_{3} \mathrm{CN}$ & $59 \%$ \\
\hline 6 & $\mathrm{AlCl}_{3}$ & $-40^{\circ} \mathrm{C}$ & $\mathrm{CH}_{3} \mathrm{CN}$ & $48 \%$ \\
\hline 7 & $\mathrm{Al}(\mathrm{OTf})_{3}$ & $-40^{\circ} \mathrm{C}$ & $\mathrm{CH}_{3} \mathrm{CN}$ & $62 \%$ \\
\hline 8 & $\mathrm{Sc}(\mathrm{OTf})_{3}$ & $-40^{\circ} \mathrm{C}$ & $\mathrm{CH}_{3} \mathrm{CN}$ & $66 \%$ \\
\hline 9 & $\mathrm{Zn}(\mathrm{OTf})_{3}$ & $-40^{\circ} \mathrm{C}$ & $\mathrm{CH}_{3} \mathrm{CN}$ & $13 \%$ \\
\hline 10 & $\ln (\mathrm{OTf})_{3}$ & $-40^{\circ} \mathrm{C}$ & $\mathrm{CH}_{3} \mathrm{CN}$ & $37 \%$ \\
\hline 11 & $\mathrm{Ga}(\mathrm{OTf})_{3}$ & $-40^{\circ} \mathrm{C}$ & $\mathrm{CH}_{3} \mathrm{CN}$ & $21 \%$ \\
\hline 12 & $\mathrm{HCl}$ & $-40^{\circ} \mathrm{C}$ & $\mathrm{CH}_{3} \mathrm{CN}$ & n.a. \\
\hline 13 & $10 \% \mathrm{BF}_{3} \cdot \mathrm{OEt}_{2}$ & $-40^{\circ} \mathrm{C}$ & $\mathrm{CH}_{3} \mathrm{CN}$ & $<10 \%$ \\
\hline 14 & $10 \% \mathrm{Sc}(\mathrm{OTf})_{3}$ & $-40^{\circ} \mathrm{C}$ & $\mathrm{CH}_{3} \mathrm{CN}$ & $<10 \%$ \\
\hline
\end{tabular}


Preparation of substrates:

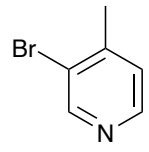

1 equiv. $\underset{\text { DMF, reflux, } 24 \mathrm{~h}}{\stackrel{1.3 \text { equivs. } \mathrm{CuCN}}{\longrightarrow}}$<smiles>Cc1ccncc1C#N</smiles>

$91 \%$

Copper (I) cyanide ( $1.16 \mathrm{~g}, 1.3$ equivs., $13 \mathrm{mmol}$ ) was added to a $50 \mathrm{~mL}$ round-bottomed flask containing 3-bromo-4-methyl-pyridine $(1.11 \mathrm{~mL}, 1.0$ equiv., $10 \mathrm{mmol})$ in anhydrous DMF (20 $\mathrm{mL}$ ), and the resulting mixture was heated at reflux for 24 hours. The reaction mixture was allowed to reach ambient temperature and then cooled to $0{ }^{\circ} \mathrm{C}$. The above reaction mixture was diluted with water $(20 \mathrm{~mL})$ while maintaining the temperature at $0^{\circ} \mathrm{C}$. An excess of aqueous $\mathrm{NH}_{4} \mathrm{OH}(28-$ $30 \%$ solution) was added until the green precipitate was completely dissolved. The above aqueous solution was extracted with diethyl ether $(3 \times 30 \mathrm{~mL})$. The combined organic layer was washed with brine, dried with $\mathrm{MgSO}_{4}$, filtered through a cotton plug, and the filtrate was concentrated using a rotary evaporator. The crude product was purified by flash column chromatography using EtOAc/hexanes to afford compound 9 as white solid (602 $\mathrm{mg}, 51 \%$ yield).

Melting point: $43.9-45.5^{\circ} \mathrm{C}$

Chromatography: $30 \%$ EtOAc in hexanes. $\left(R_{f}=0.39\right)$

1 NMR $\quad\left(400 \mathrm{MHz}, \mathrm{CDCl}_{3}\right)$

$\delta 8.79(\mathrm{~s}, 1 \mathrm{H}), 8.64(\mathrm{~d}, J=5.2 \mathrm{~Hz}, 1 \mathrm{H}), 7.28(\mathrm{~d}, J=5.2 \mathrm{~Hz}, 1 \mathrm{H}), 2.57(\mathrm{~s}, 3 \mathrm{H})$.

${ }^{13} \mathrm{C} \mathrm{NMR} \quad\left(100 \mathrm{MHz}, \mathrm{CDCl}_{3}\right)$

$\delta$ 152.9, 152.6, 151.0, 125.0, 116.0, 111.1, 20.3.

IR Alpha-Platinum ATR, Bruker, diamond crystal

$v=3023,2924,2227,1593,1406 \mathrm{~cm}^{-1}$.

HRMS $\quad$ ESI-TOF

$(\mathrm{M}+\mathrm{H})^{+}$Calcd for $\mathrm{C}_{7} \mathrm{H}_{7} \mathrm{~N}_{2}$ 119.0604; Found 119.0602. 


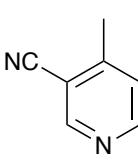

9

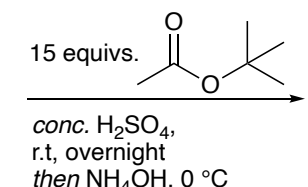

then $\mathrm{NH}_{4} \mathrm{OH}, 0^{\circ} \mathrm{C}$<smiles>Cc1ccncc1C(=O)NC(C)(C)C</smiles>

10
$96 \%$

Tert-butyl acetate (5 mL, 15 equivs., $3.75 \mathrm{mmol}$ ) and 3-cyano-4methylpyridine 9 (295.4 mg, 1.0 equiv., $2.5 \mathrm{mmol}$ ) were combined in a $25 \mathrm{~mL}$ round-bottomed flask, $1 \mathrm{~mL}$ of concentrated sulfuric acid was added dropwise, and the resulting solution was stirred at room temperature overnight. The reaction mixture was then diluted with $10 \mathrm{~mL}$ of water and carefully neutralized by dropwise addition of $30 \%$ aqueous $\mathrm{NH}_{4} \mathrm{OH}$ solution at $0{ }^{\circ} \mathrm{C}$. The organic layer was washed with brine, dried with $\mathrm{MgSO}_{4}$, filtered through a cotton plug, and the filtrate was concentrated using a rotary evaporator. The crude product was purified by flash column chromatography using EtOAc/hexanes to afford compound 10 as white solid (460 mg, 96\% yield).

Melting point: $93.6-94.7^{\circ} \mathrm{C}$

Chromatography: $70 \%$ EtOAc in hexanes. $\left(R_{f}=0.27\right)$

$\underline{{ }^{1} \mathrm{H} \text { NMR }} \quad\left(400 \mathrm{MHz}, \mathrm{CDCl}_{3}\right)$

$\delta 8.51(\mathrm{~s}, 1 \mathrm{H}), 8.44(\mathrm{~d}, J=5.0 \mathrm{~Hz}, 1 \mathrm{H}), 7.13(\mathrm{~d}, J=5.0 \mathrm{~Hz}, 1 \mathrm{H}), 5.72(\mathrm{bs}, 1 \mathrm{H}), 2.44$ $(\mathrm{s}, 3 \mathrm{H}), 1.47(\mathrm{~s}, 9 \mathrm{H})$.

${ }^{13} \mathrm{C} \mathrm{NMR} \quad\left(100 \mathrm{MHz}, \mathrm{CDCl}_{3}\right)$

$\delta 167.2,150.5,147.2,145.7,133.9,125.9,52.3,29.0,19.3$.

IR Alpha-Platinum ATR, Bruker, diamond crystal $v=3365,3022,2968,1644,1560,1451 \mathrm{~cm}^{-1}$.

HRMS ESI-TOF

$(\mathrm{M}+\mathrm{H})^{+}$Calcd for $\mathrm{C}_{11} \mathrm{H}_{17} \mathrm{~N}_{2} \mathrm{O}$ 193.1336; Found 193.1338. 


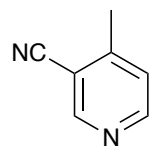

9
1 euqiv<smiles>CCOC(=O)c1cnccc1C</smiles>

11
$73 \%$

To a $25 \mathrm{~mL}$ round-bottomed flask containing 3-cyano-4-methyl-pyridine (9) (354.5 mg, 1.0 equiv., $3 \mathrm{mmol}$ ) was added $75 \%$ sulfuric acid $(6 \mathrm{~mL})$ and the resulting mixture was heated at reflux overnight. The reaction mixture was then cooled to room temperature, $15 \mathrm{~mL}$ of $95 \%$ ethanol were added, and the resulting mixture was heated at reflux for another 4 hours. The resulting mixture was cooled to room temperature and $2.0 \mathrm{M}$ aqueous $\mathrm{Na}_{2} \mathrm{CO}_{3}$ was added dropwise until the aqueous phase reached $\mathrm{pH} \sim 8$. The reaction mixture was extracted with diethyl ether $(3 \times 20$ $\mathrm{mL}$ ). The combined organic layer was washed with brine, dried with $\mathrm{MgSO}_{4}$, filtered through a cotton plug, and the filtrate was concentrated using a rotary evaporator. The crude product was purified by flash column chromatography using EtOAc/hexanes to afford compound 11 as paleyellow oil (360 mg, 73\% yield).

Chromatography: $20 \%$ EtOAc in hexanes. $\left(R_{f}=0.26\right)$

1H NMR $\quad\left(400 \mathrm{MHz}, \mathrm{CDCl}_{3}\right)$

$\delta 9.07(\mathrm{~s}, 1 \mathrm{H}), 8.55(\mathrm{~d}, J=5.1 \mathrm{~Hz}, 1 \mathrm{H}), 7.17(\mathrm{~d}, J=5.1 \mathrm{~Hz}, 1 \mathrm{H}), 4.39(\mathrm{q}, J=7.2$ $\mathrm{Hz}, 2 \mathrm{H}), 2.62(\mathrm{~s}, 3 \mathrm{H}), 1.41(\mathrm{t}, J=7.2 \mathrm{~Hz}, 3 \mathrm{H})$

${ }^{13} \mathrm{C} \mathrm{NMR} \quad\left(100 \mathrm{MHz}, \mathrm{CDCl}_{3}\right)$

$\delta 166.3,152.2,151.7,149.4,126.5,126.2,61.3,21.3,14.4$.

IR Alpha-Platinum ATR, Bruker, diamond crystal $v=3061,2981,2934,1717,1591,1405,1274 \mathrm{~cm}^{-1}$.

HRMS ESI-TOF

$(\mathrm{M}+\mathrm{H})^{+}$Calcd for $\mathrm{C}_{9} \mathrm{H}_{12} \mathrm{NO}_{2}$ 166.0863; Found 166.0866. 

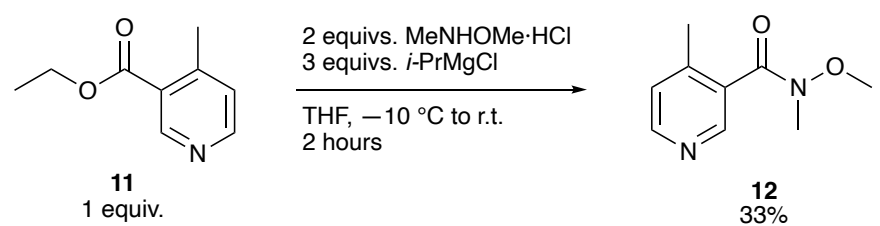

To a $50 \mathrm{~mL}$ round-bottomed flask containing pyridine 11 ( $826 \mathrm{mg}, 1.0$ equiv., $5 \mathrm{mmol}$ ) and $\mathrm{N}$, O-dimethylhydroxylamine hydrochloride (634 mg, 1.3 equivs., $6.5 \mathrm{mmol}$ ), was added $10 \mathrm{~mL}$ of anhydrous THF. The reaction mixture was cooled to $-10{ }^{\circ} \mathrm{C}$ and a solution of $i-\mathrm{PrMgCl}(7.5 \mathrm{~mL}$, $2.0 \mathrm{M}$ in THF, 3.0 equivs., $15 \mathrm{mmol}$ ) was added dropwise while stirring at $-10^{\circ} \mathrm{C}$. The reaction mixture was stirred for 1 hour at $-10^{\circ} \mathrm{C}$ and for another hour at ambient temperature. After this time the reaction was quenched by dropwise addition of saturated aqueous $\mathrm{NH}_{4} \mathrm{Cl}$ solution $(20$ $\mathrm{mL}$ ) and extracted with EtOAc $(4 \times 20 \mathrm{~mL})$. The combined organic layer was washed with brine, dried with $\mathrm{MgSO}_{4}$, filtered through a cotton plug, and the filtrate was concentrated using a rotary evaporator. The crude product was purified by flash column chromatography using $\mathrm{EtOAc/Et}{ }_{3} \mathrm{~N}$ to afford compound 12 as pale-yellow oil (297 $\mathrm{mg}, 33 \%$ yield).

Chromatography: $2.5 \% \mathrm{Et}_{3} \mathrm{~N}$ in EtOAc. $\left(\mathrm{R}_{\mathrm{f}}=0.20\right)$

1․ NMR $\quad\left(400 \mathrm{MHz}, \mathrm{CDCl}_{3}\right)$

$\delta 8.50(\mathrm{~s}, 1 \mathrm{H}), 8.48(\mathrm{~d}, J=5.1 \mathrm{~Hz}, 1 \mathrm{H}), 7.15(\mathrm{~d}, J=5.1 \mathrm{~Hz}, 1 \mathrm{H}), 3.47(\mathrm{~s}, 3 \mathrm{H}), 3.35$ $(\mathrm{s}, 3 \mathrm{H}), 2.34(\mathrm{~s}, 3 \mathrm{H})$.

13. NMR $\quad\left(100 \mathrm{MHz}, \mathrm{CDCl}_{3}\right)$

$\delta$ 168.5, 150.2, 147.1, 144.9, 131.7, 125.2, 61.5, 32.7, 19.0.

IR Alpha-Platinum ATR, Bruker, diamond crystal

$v=3064,2935,1645,1592,1446,1379,1206 \mathrm{~cm}^{-1}$.

HRMS ESI-TOF

$(\mathrm{M}+\mathrm{H})^{+}$Calcd for $\mathrm{C}_{9} \mathrm{H}_{13} \mathrm{~N}_{2} \mathrm{O}_{2}$ 181.0972; Found 181.0975. 
<smiles>Cc1ccncc1N</smiles>

1 equiv.

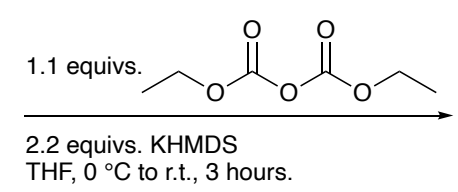

THF, $0^{\circ} \mathrm{C}$ to r.t., 3 hours.

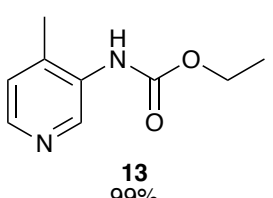

$99 \%$

To a $50 \mathrm{~mL}$ round-bottomed flask containing 3-amino-4-methyl-pyridine (216.3 mg, 1.0 equiv., $2.0 \mathrm{mmol})$ was added anhydrous THF $(20 \mathrm{~mL})$ and the resulting solution was stirred at $0{ }^{\circ} \mathrm{C}$. A solution of potassium bis(trimethylsilyl)amide ( $4.4 \mathrm{~mL}, 1.0 \mathrm{M}$ in THF, 2.2 equivs., $\left.4.4 \mathrm{mmol}\right)$ was added dropwise to the reaction mixture and stirring was continued for 30 minutes. Diethyl pyrocarbonate $(0.33 \mathrm{~mL}, 1.1$ equivs., $2.2 \mathrm{mmol})$ was added and the reaction mixture was allowed to warm to ambient temperature over three hours. The mixture was then concentrated using a rotary evaporator. The residue was dissolved in $10 \mathrm{~mL}$ of $0.1 \mathrm{M}$ aqueous $\mathrm{HCl}$, and this aqueous solution was extracted with DCM $(3 \times 20 \mathrm{~mL})$. The combined organic layer was washed with brine, dried with $\mathrm{MgSO}_{4}$, filtered through a cotton plug, and the filtrate was concentrated using a rotary evaporator. The crude product was purified by flash column chromatography using EtOAc to afford carbamate 13 as white solid (358 $\mathrm{mg}, 99 \%$ yield).

Melting point: $86.3-88.1^{\circ} \mathrm{C}$

Chromatography: $100 \%$ EtOAc. $\left(R_{f}=0.34\right)$

1․ NMR $\quad\left(400 \mathrm{MHz}, \mathrm{CDCl}_{3}\right)$

$\delta 8.88(\mathrm{~s}, 1 \mathrm{H}), 8.27(\mathrm{~d}, J=4.9 \mathrm{~Hz}, 1 \mathrm{H}), 7.10(\mathrm{~d}, J=4.9 \mathrm{~Hz}, 1 \mathrm{H}), 6.38(\mathrm{~s}, 1 \mathrm{H}), 4.24$

(q, $J=7.1 \mathrm{~Hz}, 2 \mathrm{H}$ ), 2.27 (s, 3H), $1.32(\mathrm{t}, J=7.1 \mathrm{~Hz}, 3 \mathrm{H})$.

${ }^{13} \mathrm{C}$ NMR $\quad\left(100 \mathrm{MHz}, \mathrm{CDCl}_{3}\right)$

$\delta 154.1,145.4,144.3,138.6,133.1,125.1,61.5,17.2,14.4$.

IR Alpha-Platinum ATR, Bruker, diamond crystal $v=3199,3026,2990,2932,1727,1610,1422 \mathrm{~cm}^{-1}$.

HRMS ESI-TOF

$(\mathrm{M}+\mathrm{H})^{+}$Calcd for $\mathrm{C}_{9} \mathrm{H}_{13} \mathrm{~N}_{2} \mathrm{O}_{2}$ 181.0972; Found 181.0980. 


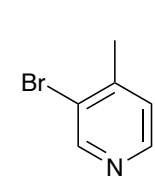

1 equiv.
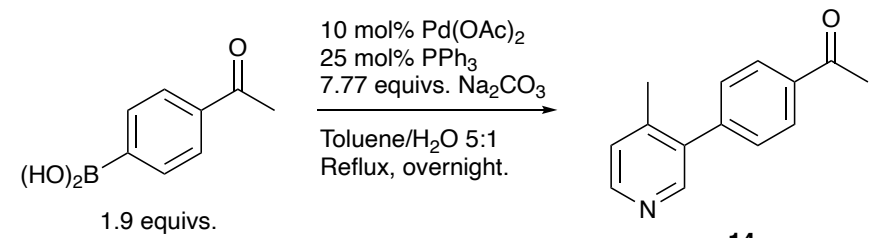

14
$98 \%$

To a $25 \mathrm{~mL}$ round-bottomed flask containing $20 \mathrm{~mL}$ of toluene and $4 \mathrm{~mL}$ of water were added palladium acetate $(89.8 \mathrm{mg}, 10 \mathrm{~mol} \%, 0.4 \mathrm{mmol})$ and triphenylphosphine $(262.3 \mathrm{mg}, 25$ $\mathrm{mol} \%, 1.0 \mathrm{mmol})$. The resulting mixture was stirred for 15 minutes at ambient temperature. 3Bromo-4-methyl-pyridine $(0.45 \mathrm{~mL}, 1.0$ equiv., $4.0 \mathrm{mmol}), p$-acetylphenylboronic acid $(1.246 \mathrm{~g}$, 1.9 equivs., $7.6 \mathrm{mmol})$ and sodium carbonate $(3.29 \mathrm{~g}, 7.77$ equivs., $31.1 \mathrm{mmol})$ were added and the resulting mixture was heated at reflux overnight. The reaction mixture was cooled to ambient temperature and filtered through a pad of celite ${ }^{\circledR}$. The filtrate was extracted with EtOAc $(4 \times 20$ $\mathrm{mL}$ ). The combined organic layer was washed with brine, dried with $\mathrm{MgSO}_{4}$, filtered through a cotton plug, and the filtrate was concentrated using a rotary evaporator. The crude product was purified by flash column chromatography using EtOAc/hexanes to afford compound 14 as white solid (831 $\mathrm{mg}, 98 \%$ yield).

Melting point: $75.1-77.0^{\circ} \mathrm{C}$

Chromatography: $60 \%$ EtOAc in hexanes. $\left(R_{f}=0.30\right)$

1H NMR $\quad\left(400 \mathrm{MHz}, \mathrm{CDCl}_{3}\right)$

$\delta 8.48(\mathrm{~d}, J=5.0 \mathrm{~Hz}, 1 \mathrm{H}), 8.43(\mathrm{~s}, 1 \mathrm{H}), 8.04(\mathrm{~d}, J=8.1 \mathrm{~Hz}, 2 \mathrm{H}), 7.42(\mathrm{~d}, J=8.1$ $\mathrm{Hz}, 2 \mathrm{H}), 7.21(\mathrm{~d}, J=5.0 \mathrm{~Hz}, 1 \mathrm{H}), 2.65(\mathrm{~s}, 3 \mathrm{H}), 2.29(\mathrm{~s}, 3 \mathrm{H})$.

${ }^{13} \mathrm{C} \mathrm{NMR} \quad\left(100 \mathrm{MHz}, \mathrm{CDCl}_{3}\right)$

$\delta 197.8,149.8,149.1,144.5,143.0,136.8,136.4,129.7,128.6,125.4,26.8,19.9$.

IR Alpha-Platinum ATR, Bruker, diamond crystal $v=3044,2970,2924,1681,1605,1269 \mathrm{~cm}^{-1}$.

HRMS $\quad$ ESI-TOF

$(\mathrm{M}+\mathrm{H})^{+}$Calcd for $\mathrm{C}_{14} \mathrm{H}_{14} \mathrm{NO} 212.1070$; Found 212.1070. 
<smiles>Cc1ccncc1Br</smiles>

1 equiv.
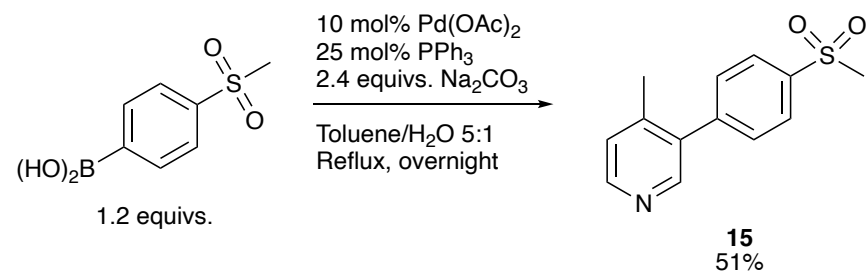

To a $25 \mathrm{~mL}$ round-bottomed flask containing $10 \mathrm{~mL}$ of toluene and $2 \mathrm{~mL}$ of water were added palladium acetate $(23 \mathrm{mg}, 10 \mathrm{~mol} \%, 0.1 \mathrm{mmol})$ and triphenylphosphine $(65.6 \mathrm{mg}, 25 \mathrm{~mol} \%$, $0.25 \mathrm{mmol}$ ). The resulting mixture was stirred for 15 minutes at ambient temperature. 3-Bromo4-methyl-pyridine ( $0.11 \mathrm{~mL}, 1.0$ equiv., $1.0 \mathrm{mmol})$, 4-(methanesulfonyl)-phenylboronic acid (240.0 $\mathrm{mg}, 1.2$ equivs., $1.2 \mathrm{mmol}$ ) and sodium carbonate $(254.4 \mathrm{mg}, 2.4$ equivs., $2.4 \mathrm{mmol})$ were added, and the resulting solution was heated at reflux overnight. The reaction mixture was cooled to ambient temperature and filtered through a pad of celite ${ }^{\circledR}$. The filtrate was extracted with EtOAc $(4 \times 20 \mathrm{~mL})$. The combined organic layer was washed with brine, dried with $\mathrm{MgSO}_{4}$, filtered through a cotton plug, and the filtrate was concentrated using a rotary evaporator. The crude product was purified by flash column chromatography using EtOAc/hexanes to afford compound 15 as white solid ( $125 \mathrm{mg}, 51 \%$ yield).

Melting point: $129.3-130.3^{\circ} \mathrm{C}$

Chromatography: $90 \%$ EtOAc in hexanes. $\left(R_{f}=0.26\right)$

$\underline{{ }^{1} \mathrm{H} \mathrm{NMR}} \quad\left(400 \mathrm{MHz}, \mathrm{CDCl}_{3}\right)$

$\delta 8.51(\mathrm{~d}, J=5.0 \mathrm{~Hz}, 1 \mathrm{H}), 8.42(\mathrm{~s}, 1 \mathrm{H}), 8.04(\mathrm{~d}, J=8.5 \mathrm{~Hz}, 2 \mathrm{H}), 7.54(\mathrm{~d}, J=8.5$ $\mathrm{Hz}, 2 \mathrm{H}), 7.24$ (d, J=5.0 Hz, 1H), $3.13(\mathrm{~s}, 3 \mathrm{H}), 2.29$ (s, 3H).

${ }^{13} \mathbf{C ~ N M R} \quad\left(100 \mathrm{MHz}, \mathrm{CDCl}_{3}\right)$

$\delta 149.7,149.5,144.5,143.9,140.0,136.1,130.4,127.8,125.5,44.7,19.9$.

IR $\quad$ Alpha-Platinum ATR, Bruker, diamond crystal

$v=3039,3017,2926,1590,1327,1038 \mathrm{~cm}^{-1}$.

HRMS ESI-TOF

$(\mathrm{M}+\mathrm{H})^{+}$Calcd for $\mathrm{C}_{13} \mathrm{H}_{14} \mathrm{NO}_{2} \mathrm{~S} 248.0740$; Found 248.0741. 


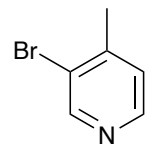

1 equiv.

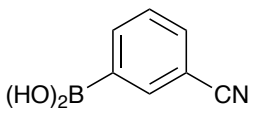

1.9 equivs.

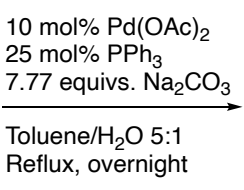

Reflux, overi

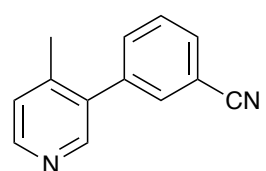

16
$92 \%$

To a $50 \mathrm{~mL}$ round-bottomed flask containing $20 \mathrm{~mL}$ of toluene and $4 \mathrm{~mL}$ of water were added palladium acetate (45 mg, $10 \mathrm{~mol} \%, 0.2 \mathrm{mmol}$ ) and triphenylphosphine (131.1 $\mathrm{mg}, 25$ $\mathrm{mol} \%, 0.5 \mathrm{mmol})$. The resulting mixture was stirred for 15 minutes at ambient temperature. 3Bromo-4-methyl-pyridine ( $0.22 \mathrm{~mL}, 1.0$ equiv., $2.0 \mathrm{mmol})$, 3-cyanophenylboronic acid (558.2 $\mathrm{mg}$, 1.9 equivs., $3.8 \mathrm{mmol}$ ) and sodium carbonate (1.65 g, 7.77 equivs., $15.5 \mathrm{mmol}$ ) were added, and the resulting mixture was heated at reflux overnight. The reaction mixture was cooled to ambient temperature and filtered through a pad of celite ${ }^{\circledR}$. The filtrate was extracted with EtOAc $(4 \times 20$ $\mathrm{mL}$ ). The combined organic layer was washed with brine, dried with $\mathrm{MgSO}_{4}$, filtered through a cotton plug, and the filtrate was concentrated using a rotary evaporator. The crude product was purified by flash column chromatography using EtOAc/hexanes/Et ${ }_{3} \mathrm{~N}$ to afford compound 16 as white solid (358 $\mathrm{mg}, 92 \%$ yield).

Melting point: $63.4-64.1^{\circ} \mathrm{C}$

Chromatography: $40 \%$ EtOAc in hexanes with $2 \% \mathrm{Et}_{3} \mathrm{~N} .\left(\mathrm{R}_{\mathrm{f}}=0.24\right)$

$\underline{{ }^{1} \mathrm{H} \mathrm{NMR}} \quad\left(400 \mathrm{MHz}, \mathrm{CDCl}_{3}\right)$

$\delta 8.44(\mathrm{~d}, J=5.1 \mathrm{~Hz}, 1 \mathrm{H}), 8.34(\mathrm{~s}, 1 \mathrm{H}), 7.66-7.63(\mathrm{~m}, 1 \mathrm{H}), 7.58-7.56(\mathrm{~m}, 1 \mathrm{H})$, $7.55-7.50(\mathrm{~m}, 2 \mathrm{H}), 7.17(\mathrm{~d}, J=5.1 \mathrm{~Hz}, 1 \mathrm{H}), 2.23(\mathrm{~s}, 3 \mathrm{H})$.

${ }^{13} \mathrm{C}$ NMR $\quad\left(100 \mathrm{MHz}, \mathrm{CDCl}_{3}\right)$

$\delta 149.6,149.2,144.3,139.2,135.5,133.7,132.6,131.3,129.4,125.3,118.4$, $112.7,19.6$.

IR Alpha-Platinum ATR, Bruker, diamond crystal $v=3026,2956,2854,2227,1592,790 \mathrm{~cm}^{-1}$.

HRMS ESI-TOF

$(\mathrm{M}+\mathrm{H})^{+}$Calcd for $\mathrm{C}_{13} \mathrm{H}_{11} \mathrm{~N}_{2}$ 195.0917; Found 195.0917. 


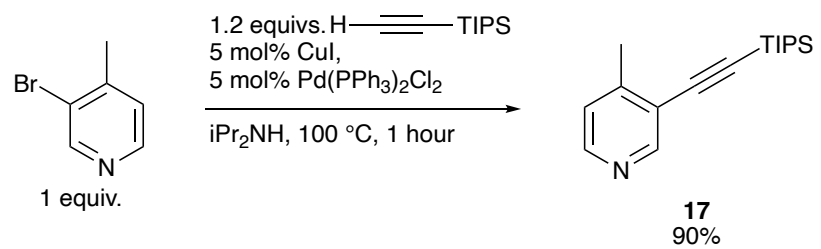

To a $50 \mathrm{~mL}$ round-bottomed flask containing $24 \mathrm{~mL}$ of $i-\mathrm{Pr}_{2} \mathrm{NH}$, 3-bromo-4-methyl-pyridine (0.58 mL, 1.0 equiv., $5.0 \mathrm{mmol})$ and triisopropylacetylene $(1.35 \mathrm{mg}, 1.2$ equivs., $6.0 \mathrm{mmol})$ were added copper (I) iodide (48 mg, $5 \mathrm{~mol} \%$., $0.25 \mathrm{mmol}$ ) and bis(triphenylphosphine)-palladium (II) dichloride $(175.5 \mathrm{mg}, 5 \mathrm{~mol} \%, 0.25 \mathrm{mmol})$. The resulting mixture was heated at reflux for 1 hour and then cooled to ambient temperature. The reaction mixture was diluted with $100 \mathrm{~mL}$ of EtOAc and the resulting suspension was filtered through celite ${ }^{\circledR}$. The filtrate was concentrated using a rotary evaporator. The crude product was purified by flash column chromatography using EtOAC/hexanes to afford compound 17 as colorless oil ( $1.24 \mathrm{~g}, 90 \%$ yield).

Chromatography: $10 \%$ EtOAc in hexanes. $\left(R_{f}=0.31\right)$

1 NMR $\left(400 \mathrm{MHz}, \mathrm{CDCl}_{3}\right)$

$\delta 8.58(\mathrm{~s}, 1 \mathrm{H}), 8.33(\mathrm{~d}, J=5.1 \mathrm{~Hz}, 1 \mathrm{H}), 7.07(\mathrm{~d}, J=5.1 \mathrm{~Hz}, 1 \mathrm{H}), 2.41(\mathrm{~s}, 3 \mathrm{H}), 1.11$ $(\mathrm{s}, 21 \mathrm{H})$.

${ }^{13} \mathrm{C} \mathrm{NMR} \quad\left(100 \mathrm{MHz}, \mathrm{CDCl}_{3}\right)$

$\delta 152.8,149.1,148.4,124.1,121.0,102.4,98.5,20.4,18.7,11.3$.

IR Alpha-Platinum ATR, Bruker, diamond crystal

$v=3037,2941,2891,2154,1584,1461,1225 \mathrm{~cm}^{-1}$.

HRMS ESI-TOF

$(\mathrm{M}+\mathrm{H})^{+}$Calcd forC ${ }_{17} \mathrm{H}_{28} \mathrm{NSi} 274.1986$; Found 274.2000. 

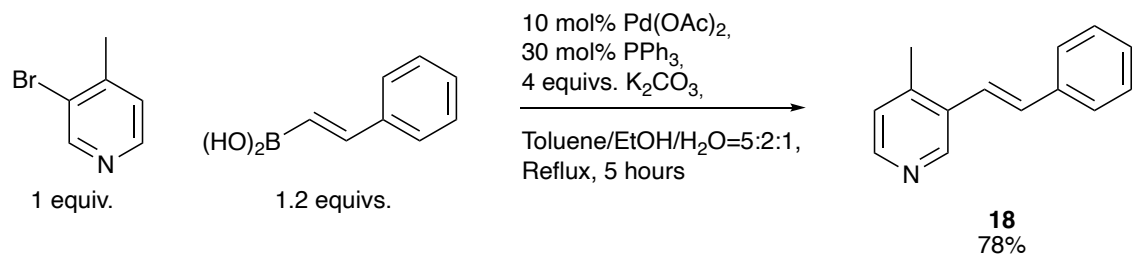

To a $100 \mathrm{~mL}$ round-bottomed flask containing $34 \mathrm{~mL}$ of toluene/ethanol/water (5:2:1) and 3-bromo-4-methyl-pyridine $(0.2 \mathrm{~mL}, 1.0$ equiv., $1.7 \mathrm{mmol})$ were added trans-2-phenylvinylboronic acid $(295.9 \mathrm{mg}, 1.2$ equivs., $2.0 \mathrm{mmol})$, palladium acetate $(38.1 \mathrm{mg}, 10 \mathrm{~mol} \%, 0.17 \mathrm{mmol})$, triphenylphosphine $(133.8 \mathrm{mg}, 30 \mathrm{~mol} \%, 0.51 \mathrm{mmol})$ and potassium carbonate $(939.8 \mathrm{mg}, 4.0$ equivs., $6.8 \mathrm{mmol}$ ). The resulting mixture was stirred and heated at reflux for 5 hours and then cooled to ambient temperature. Ethanol was then removed using a rotary evaporator. The residue was extracted with EtOAc $(3 \times 20 \mathrm{~mL})$. The combined organic layer was washed with brine, dried with $\mathrm{MgSO}_{4}$, filtered through a cotton plug, and the filtrate was concentrated using a rotary evaporator. The crude product was purified by flash column chromatography using EtOAc/hexanes to afford compound 18 as light-yellow oil (259mg, 78\% yield).

Chromatography: $30 \%$ EtOAc in hexanes. $\left(R_{f}=0.23\right)$

1'H NMR $\quad\left(400 \mathrm{MHz}, \mathrm{CDCl}_{3}\right)$

$\delta 8.74(\mathrm{~s}, 1 \mathrm{H}), 8.36(\mathrm{~d}, J=4.9 \mathrm{~Hz}, 1 \mathrm{H}), 7.52(\mathrm{~d}, J=7.5 \mathrm{~Hz}, 2 \mathrm{H}), 7.40-7.35(\mathrm{~m}$, $2 \mathrm{H}), 7.32-7.27(\mathrm{~m}, 1 \mathrm{H}), 7.21(\mathrm{~d}, J=16.2 \mathrm{~Hz}, 1 \mathrm{H}), 7.09-7.02(\mathrm{~m}, 2 \mathrm{H}), 2.40(\mathrm{~s}$, $3 \mathrm{H})$.

${ }^{13} \mathrm{C} \mathrm{NMR} \quad\left(100 \mathrm{MHz}, \mathrm{CDCl}_{3}\right)$

$\delta 148.3,147.0,144.3,137.1,132.9,132.0,128.9,128.2,126.8,125.1,123.3,19.4$.

IR Alpha-Platinum ATR, Bruker, diamond crystal $v=3025,2974,2921,1587,1447,960 \mathrm{~cm}^{-1}$.

HRMS ESI-TOF

$(\mathrm{M}+\mathrm{H})^{+}$Calcd for $\mathrm{C}_{14} \mathrm{H}_{14} \mathrm{~N}$ 196.1121; Found 196.1123. 


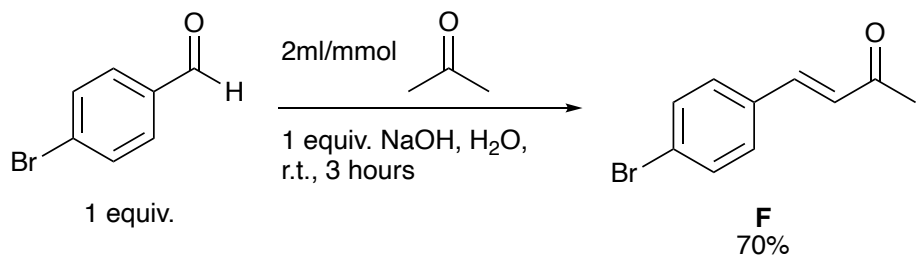

To a solution of 4-bromobenzaldehyde (925 mg, 1.0 equiv., $5 \mathrm{mmol}$ ) in $10 \mathrm{~mL}$ of acetone was added $5 \mathrm{~mL}$ of $1 \mathrm{M}$ aqueous $\mathrm{NaOH}$, and the resulting mixture was stirred at ambient temperature for 3 hours. The reaction mixture was cooled to $0{ }^{\circ} \mathrm{C}$ and neutralized by dropwise addition of $1 \mathrm{M}$ aqueous $\mathrm{HCl}$. The resulting mixture was concentrated using a rotary evaporator to remove excess acetone. The residue was then extracted with EtOAc $(4 \times 20 \mathrm{~mL})$. The combined organic layer was washed with brine, dried with $\mathrm{MgSO}_{4}$, filtered through a cotton plug, and the filtrate was concentrated using a rotary evaporator. The crude product was purified by flash column chromatography using EtOAc/hexanes to afford compound $\mathbf{F}$ as white solid (782 $\mathrm{mg}$, $70 \%)$. The NMR data matches the literature values. The NMR data collected matches the data reported in the literature. ${ }^{1}$

Chromatography: $10 \%$ EtOAc in hexanes. $\left(R_{f}=0.21\right)$

$\underline{{ }^{1} \mathrm{H} \text { NMR }} \quad\left(300 \mathrm{MHz}, \mathrm{CDCl}_{3}\right)$

$\delta 7.54-7.51(\mathrm{~m}, 2 \mathrm{H}), 7.47-7.38(\mathrm{~m}, 3 \mathrm{H}), 6.69(\mathrm{~d}, J=16.3 \mathrm{~Hz}, 1 \mathrm{H}), 2.37(\mathrm{~s}, 3 \mathrm{H})$.

${ }^{13} \mathrm{C} \mathrm{NMR} \quad\left(75 \mathrm{MHz}, \mathrm{CDCl}_{3}\right)$

$\delta 198.2,142.0,133.4,132.4,129.7,127.7,124.9,27.8$.

${ }^{1}$ H. Brunner, T. Stern, S. Rückbrod, C. Czekelius and C. Donner, Adv. Synth. Catal., 2010, 352, 1983. 


\section{Conjugate addition of alkylidene dihydropyridines to $\alpha, \beta$-unsaturated ketones:}
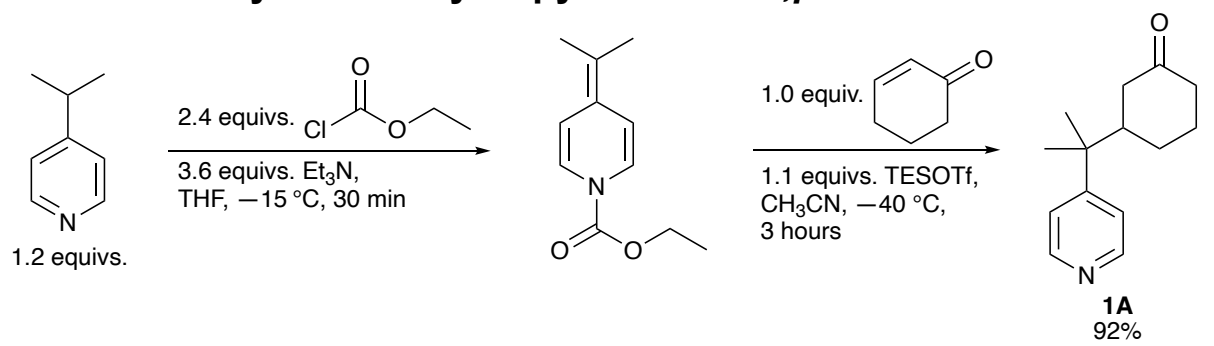

To a dry round-bottomed flask containing anhydrous THF were added 4-isopropyl-pyridine (145.4 mg, 1.2 equivs., $1.2 \mathrm{mmol})$ and triethylamine $(0.50 \mathrm{~mL}, 3.6$ equivs., $3.6 \mathrm{mmol})$. The resulting solution was cooled to $-15^{\circ} \mathrm{C}$. A solution of ethyl chloroformate $(0.23 \mathrm{~mL}, 2.4$ equivs., $2.4 \mathrm{mmol}$ ) in $5.7 \mathrm{~mL}$ of anhydrous THF was added to the above mixture using a syringe pump over 30 minutes while maintaining the temperature at $-15^{\circ} \mathrm{C}$. The reaction mixture was allowed to warm to ambient temperature and then concentrated using a rotary evaporator. The residue was dissolved in diethyl ether, triturated for 2-3 minutes and filtered through a cotton plug to remove the precipitated triethylammonium chloride salt. The filtrate was then concentrated using a rotary evaporator, and the corresponding ADHP was obtained and used immediately for next step without further purification.

Final product $1 \mathrm{~A}$ was synthesized following general procedure B using 2-cyclohexen-1one $(0.1 \mathrm{~mL}, 1.0$ equiv. $1.0 \mathrm{mmol})$ as the electrophile. The crude product was purified by flash column chromatography using EtOAc/hexanes to afford compound $\mathbf{1 A}$ as white solid (201 mg, $92 \%$ yield).

Melting point: $82.1-83.1^{\circ} \mathrm{C}$.

Chromatography: $70 \%$ EtOAc in hexanes. $\left(R_{f}=0.29\right)$

1 NMR $\quad\left(300 \mathrm{MHz}, \mathrm{CDCl}_{3}\right)$ :

$\delta 8.52(\mathrm{~d}, J=6.2 \mathrm{~Hz}, 2 \mathrm{H}), 7.18(\mathrm{~d}, J=6.2 \mathrm{~Hz}, 2 \mathrm{H}), 2.38-2.11(\mathrm{~m}, 3 \mathrm{H}), 2.08-$ $1.87(\mathrm{~m} .3 \mathrm{H}), 1.73-1.62(\mathrm{~m}, 1 \mathrm{H}), 1.58-1.41(\mathrm{~m}, 1 \mathrm{H}), 1.35-1.21(\mathrm{~m}, 7 \mathrm{H})$.

${ }^{13} \mathrm{C} \mathrm{NMR} \quad\left(75 \mathrm{MHz}, \mathrm{CDCl}_{3}\right)$

$\delta$ 211.6, 157.4, 150.0, 121.6, 49.2, 43.7, 41.3, 40.5, 26.5, 25.4, 24.9, 24.1.

IR Alpha-Platinum ATR, Bruker, diamond crystal $v=3022,2965,2934,1705,1597,1413 \mathrm{~cm}^{-1}$.

HRMS ESI-TOF

$(\mathrm{M}+\mathrm{H})^{+}$Calcd for $\mathrm{C}_{14} \mathrm{H}_{20} \mathrm{NO}$ 218.1540; Found 218.1545. 

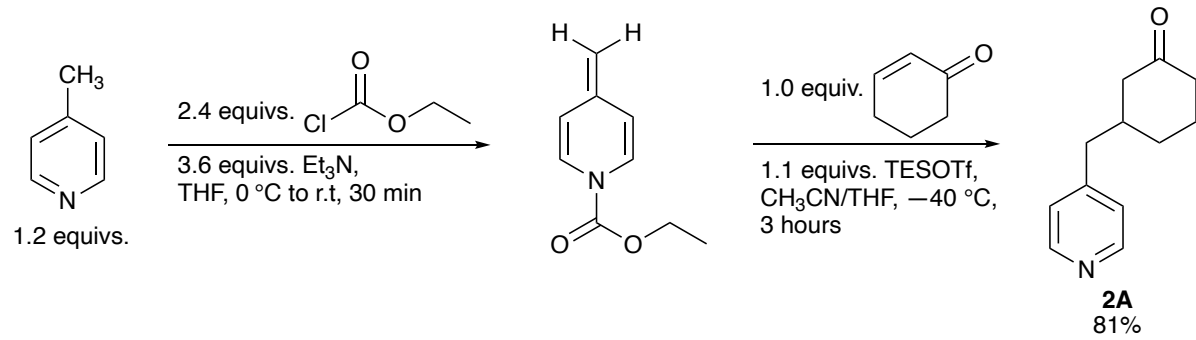

4-Picoline $(0.12 \mathrm{~mL}, 1.2$ equivs., $1.2 \mathrm{mmol})$ was converted to the corresponding ADHP following general procedure A. Final product 2A was synthesized following general procedure B but using anhydrous THF $(0.2 \mathrm{M})$ to dissolve the ADHP instead of $\mathrm{CH}_{3} \mathrm{CN}$ due to its poor solubility in $\mathrm{CH}_{3} \mathrm{CN}$, and 2-cyclohexen-1-one $(0.1 \mathrm{~mL}, 1.0$ equiv. $1.0 \mathrm{mmol})$ as the electrophile. The crude product was purified by flash column chromatography using EtOAc/hexanes/Et ${ }_{3} \mathrm{~N}$ to afford compound $2 \mathrm{~A}$ as colorless oil (154 $\mathrm{mg}, 81 \%$ yield).

Chromatography: $70 \%$ EtOAc in hexanes with $2 \% \mathrm{Et}_{3} \mathrm{~N} .\left(\mathrm{R}_{\mathrm{f}}=0.37\right)$

${ }^{1} \mathrm{H}$ NMR $\quad\left(400 \mathrm{MHz}, \mathrm{CDCl}_{3}\right)$

$\delta 8.49(\mathrm{~d}, J=5.8 \mathrm{~Hz}, 2 \mathrm{H}), 7.04(\mathrm{~d}, J=5.8 \mathrm{~Hz}, 2 \mathrm{H}), 2.65-2.55(\mathrm{~m}, 2 \mathrm{H}), 2.37-$

$2.31(\mathrm{~m}, 2 \mathrm{H}), 2.29-2.20(\mathrm{~m}, 1 \mathrm{H}), 2.13-1.99(\mathrm{~m}, 3 \mathrm{H}), 1.87-1.82(\mathrm{~m}, 1 \mathrm{H}), 1.67$

$-1.55(\mathrm{~m}, 1 \mathrm{H}), 1.42-1.32(\mathrm{~m}, 1 \mathrm{H})$.

${ }^{13} \mathrm{C} \mathrm{NMR} \quad\left(100 \mathrm{MHz}, \mathrm{CDCl}_{3}\right)$

$\delta 210.7,149.9,148.4,124.5,47.7,42.3,41.3,40.0,30.9,25.0$.

IR Alpha-Platinum ATR, Bruker, diamond crystal

$v=3022,2934,2867,1706,1601,1416 \mathrm{~cm}^{-1}$.

HRMS ESI-TOF

$(\mathrm{M}+\mathrm{H})^{+}$Calcd for $\mathrm{C}_{12} \mathrm{H}_{16} \mathrm{NO}$ 190.1227; Found 190.1233. 

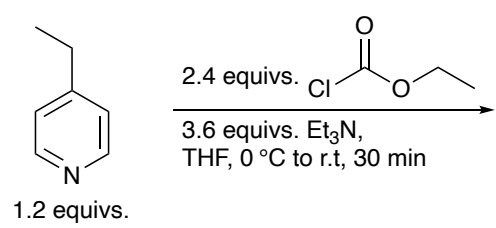
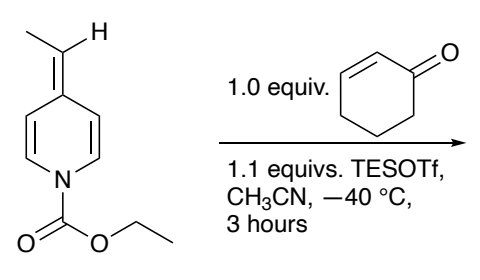

4-Ethylpyridine $(0.14 \mathrm{~mL}, 1.2$ equivs., $1.2 \mathrm{mmol})$ was converted to the corresponding ADHP following general procedure $\mathbf{A}$. Final product $3 \mathbf{A}$ was synthesized using general procedure $\mathbf{B}$ with 2-cyclohexen-1-one $(0.1 \mathrm{~mL}, 1.0$ equiv. $1.0 \mathrm{mmol})$ as the electrophile. The mixture of diastereomers was separated by flash column chromatography using $\mathrm{MeCN} / \mathrm{DCM} / \mathrm{hexanes}$ to afford $111 \mathrm{mg}$ of major diastereomer (3A-1) as colorless oil and $69 \mathrm{mg}$ of minor diastereomer (3A-2) as colorless oil, in 89\% combined yield (d.r. 2:1).

Data for major diastereomer (3A-1):

Chromatography: $14 \%$ MeCN, 36\% DCM with $50 \%$ hexanes

1H NMR $\quad\left(400 \mathrm{MHz}, \mathrm{CDCl}_{3}\right)$

$\delta 8.52-8.40(\mathrm{~m}, 2 \mathrm{H}), 7.05(\mathrm{~d}, J=4.6 \mathrm{~Hz}, 2 \mathrm{H}), 2.62-2.54(\mathrm{~m}, 1 \mathrm{H}), 2.54-2.50$

$(\mathrm{m}, 1 \mathrm{H}), 2.36-2.31(\mathrm{~m}, 1 \mathrm{H}), 2.25-2.16(\mathrm{~m}, 1 \mathrm{H}), 2.08-2.01(\mathrm{~m}, 1 \mathrm{H}), 2.01-1.88$

$(\mathrm{m}, 2 \mathrm{H}), 1.65-1.61(\mathrm{~m}, 1 \mathrm{H}), 1.58-1.45(\mathrm{~m}, 1 \mathrm{H}), 1.26-1.16(\mathrm{~m}, 4 \mathrm{H})$.

${ }^{13} \mathrm{C} \mathrm{NMR} \quad\left(100 \mathrm{MHz}, \mathrm{CDCl}_{3}\right)$

$\delta 211.2,154.2,150.0,123.1,45.8,45.0,44.7,41.4,29.4,25.1,18.1$.

Data for minor diastereomer (3A-2):

Chromatography: $14 \% \mathrm{MeCN}, 36 \%$ DCM with $50 \%$ hexanes

1 NMR $\quad\left(400 \mathrm{MHz}, \mathrm{CDCl}_{3}\right)$

$\delta 8.52-8.51(\mathrm{~m}, 2 \mathrm{H}), 7.05(\mathrm{~d}, J=5.4 \mathrm{~Hz}, 2 \mathrm{H}), 2.61(\mathrm{p}, J=6.9 \mathrm{~Hz}, 1 \mathrm{H}), 2.37-$ $2.32(\mathrm{~m}, 1 \mathrm{H}), 2.26-2.20(\mathrm{~m}, 1 \mathrm{H}), 2.19-2.15(\mathrm{~m}, 1 \mathrm{H}), 2.12-2.07(\mathrm{~m}, 1 \mathrm{H}), 2.06$

$-2.00(\mathrm{~m}, 1 \mathrm{H}), 1.98-1.87(\mathrm{~m}, 2 \mathrm{H}), 1.69-1.57(\mathrm{~m}, 1 \mathrm{H}), 1.43-1.33(\mathrm{~m}, 1 \mathrm{H}), 1.29$

(d, $J=7.0 \mathrm{~Hz}, 3 \mathrm{H})$.

${ }^{13} \mathrm{C}$ NMR $\quad\left(100 \mathrm{MHz}, \mathrm{CDCl}_{3}\right)$

$\delta 211.2,153.8,150.1,123.2,46.2,44.8,44.7,41.4,28.8,25.1,18.1$.

IR Alpha-Platinum ATR, Bruker, diamond crystal

$v=3062,2935,2870,1705,1600,1416 \mathrm{~cm}^{-1}$.

HRMS ESI-TOF

$(\mathrm{M}+\mathrm{H})^{+}$Calcd for $\mathrm{C}_{13} \mathrm{H}_{18} \mathrm{NO} 204.1383$; Found 204.1391. 

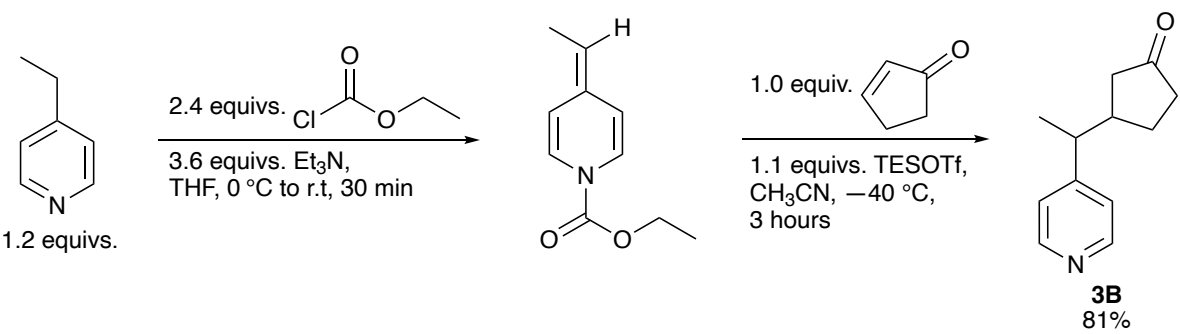

4-Ethylpyridine $(0.14 \mathrm{~mL}, 1.2$ equivs., $1.2 \mathrm{mmol})$ was converted to the corresponding ADHP following general procedure $\mathbf{A}$. Final product $3 \mathbf{B}$ was synthesized using general procedure $\mathbf{B}$ with 2-cyclopenten-1-one $(0.1 \mathrm{~mL}, 1.0$ equiv. $1.0 \mathrm{mmol})$ as the electrophile. The mixture of diastereomers was separated by flash column chromatography using MeCN/DCM/hexanes to afford $102 \mathrm{mg}$ of major diastereomer (3B-1) as a colorless oil and $51 \mathrm{mg}$ of minor diastereomer (3B-2) as colorless oil, in 81\% combined yield (d.r. $\sim 2: 1$ ).

Data for major diastereomer (3B-1):

Chromatography: $14 \% \mathrm{MeCN}, 36 \%$ DCM with $50 \%$ hexanes

$\underline{{ }^{1} \mathrm{H} \text { NMR }} \quad\left(400 \mathrm{MHz}, \mathrm{CDCl}_{3}\right)$

$\delta 8.51(\mathrm{~d}, J=5.9 \mathrm{~Hz}, 2 \mathrm{H}), 7.10(\mathrm{~d}, J=5.9 \mathrm{~Hz}, 2 \mathrm{H}), 2.59-2.47(\mathrm{~m}, 2 \mathrm{H}), 2.36-$ $2.21(\mathrm{~m}, 2 \mathrm{H}), 2.12-2.02(\mathrm{~m}, 1 \mathrm{H}), 1.96-1.88(\mathrm{~m}, 1 \mathrm{H}), 1.78-1.70(\mathrm{~m}, 1 \mathrm{H}), 1.44$

$-1.32(\mathrm{~m}, 1 \mathrm{H}), 1.28(\mathrm{~d}, J=6.9 \mathrm{~Hz}, 3 \mathrm{H})$.

${ }^{13} \mathrm{C} \mathrm{NMR} \quad\left(100 \mathrm{MHz}, \mathrm{CDCl}_{3}\right)$

$\delta 218.2,154.3,150.1,122.8,45.6,44.0,43.7,38.9,28.5,20.4$.

Data for minor diastereomer (3B-2):

Chromatography: $14 \% \mathrm{MeCN}, 36 \%$ DCM with $50 \%$ hexanes

1 NMR $\quad\left(400 \mathrm{MHz}, \mathrm{CDCl}_{3}\right)$

$\delta 8.55-8.53(\mathrm{~m}, 2 \mathrm{H}), 7.10(\mathrm{~d}, J=6.0 \mathrm{~Hz}, 2 \mathrm{H}), 2.65-2.56(\mathrm{~m}, 1 \mathrm{H}), 2.42-2.28(\mathrm{~m}$, $3 \mathrm{H}), 2.26-2.16(\mathrm{~m}, 1 \mathrm{H}), 2.09-2.02(\mathrm{~m}, 1 \mathrm{H}), 1.79-1.70(\mathrm{~m}, 1 \mathrm{H}), 1.69-1.58(\mathrm{~m}$, $1 \mathrm{H}), 1.35(\mathrm{~d}, J=6.9 \mathrm{~Hz}, 3 \mathrm{H})$

${ }^{13} \mathbf{C ~ N M R} \quad\left(100 \mathrm{MHz}, \mathrm{CDCl}_{3}\right)$

$\delta 218.1,154.8,150.2,122.7,45.4,44.2,43.6,38.9,28.2,19.4$.

IR Alpha-Platinum ATR, Bruker, diamond crystal $v=3030,2934,2870,1706,1598,1416 \mathrm{~cm}^{-1}$.

HRMS ESI-TOF

$(\mathrm{M}+\mathrm{H})^{+}$Calcd for $\mathrm{C}_{12} \mathrm{H}_{16} \mathrm{NO}$ 190.1227; Found 190.1234. 


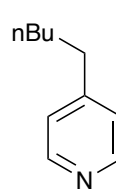

1.2 equivs
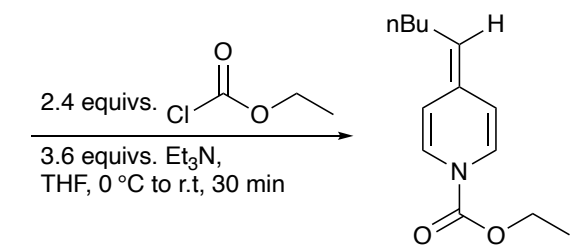
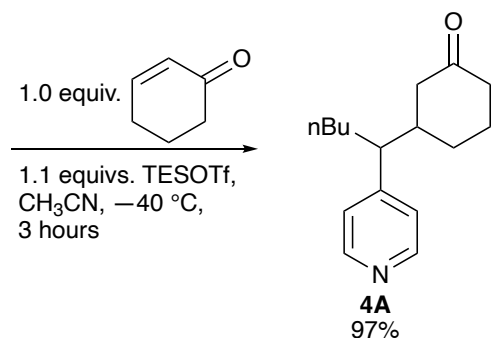

4- $n$-Pentyl-pyridine (179.1 mg, 1.2 equivs., $1.2 \mathrm{mmol}$ ) was transformed to the corresponding ADHP following general procedure A. Final product 4A was synthesized using general procedure $\mathbf{B}$ with 2-cyclohexen-1-one $(0.1 \mathrm{~mL}, 1.0$ equiv. $1.0 \mathrm{mmol})$ as the electrophile. The mixture of diastereomers was separated by flash column chromatography using $\mathrm{MeCN} / \mathrm{DCM} /$ hexanes to afford $124 \mathrm{mg}$ of diastereomer (4A-1) as colorless oil and $133 \mathrm{mg}$ of diastereomer (4A-2) as colorless oil, in 97\% combined yield (d.r. $\sim 1.1$ ).

Data for diastereomer (4A-1):

Chromatography: $14 \%$ MeCN, 36\% DCM with $50 \%$ hexanes $\left(R_{f}=0.35\right)$

1H NMR $\quad\left(400 \mathrm{MHz}, \mathrm{CDCl}_{3}\right)$

$\delta 8.52(\mathrm{~d}, J=5.6 \mathrm{~Hz}, 2 \mathrm{H}), 7.04(\mathrm{~d}, J=5.6 \mathrm{~Hz}, 2 \mathrm{H}), 2.59-2.52(\mathrm{~m}, 1 \mathrm{H}), 2.43-$ $2.38(\mathrm{~m}, 1 \mathrm{H}), 2.36-2.31(\mathrm{~m}, 1 \mathrm{H}), 2.25-2.14(\mathrm{~m}, 1 \mathrm{H}), 2.06-1.95(\mathrm{~m}, 3 \mathrm{H}), 1.77$

$-1.71(\mathrm{~m}, 1 \mathrm{H}), 1.66-1.45(\mathrm{~m}, 3 \mathrm{H}), 1.32-1.16(\mathrm{~m}, 3 \mathrm{H}), 1.10-0.98(\mathrm{~m}, 2 \mathrm{H}), 0.80$ $(\mathrm{t}, J=7.3 \mathrm{~Hz}, 3 \mathrm{H})$.

${ }^{13} \mathrm{C} \mathrm{NMR} \quad\left(100 \mathrm{MHz}, \mathrm{CDCl}_{3}\right)$

$\delta 211.2,152.4,150.0,124.1,51.2,46.7,43.9,41.5,31.8,29.7,29.0,25.1,22.7$, 14.0 .

Data for diastereomer (4A-2):

Chromatography: $14 \%$ MeCN, 36\% DCM with $50 \%$ hexanes $\left(R_{f}=0.28\right)$

1H NMR $\quad\left(400 \mathrm{MHz}, \mathrm{CDCl}_{3}\right)$

$\delta 8.52(\mathrm{~d}, J=6.0 \mathrm{~Hz}, 2 \mathrm{H}), 7.02(\mathrm{~d}, J=6.0 \mathrm{~Hz}, 2 \mathrm{H}), 2.49-2.45(\mathrm{~m}, 1 \mathrm{H}), 2.37-$

$2.31(\mathrm{~m}, 1 \mathrm{H}), 2.21-2.14(\mathrm{~m}, 2 \mathrm{H}), 2.10-1.95(\mathrm{~m}, 3 \mathrm{H}), 1.90-1.83(\mathrm{~m}, 1 \mathrm{H}), 1.83$

$-1.75(\mathrm{~m}, 1 \mathrm{H}), 1.70-1.55(\mathrm{~m}, 2 \mathrm{H}), 1.39-1.15(\mathrm{~m}, 3 \mathrm{H}), 1.12-0.95(\mathrm{~m}, 2 \mathrm{H}), 0.82$

$(\mathrm{t}, J=7.3 \mathrm{~Hz}, 3 \mathrm{H})$.

${ }^{13} \mathrm{C}$ NMR $\quad\left(100 \mathrm{MHz}, \mathrm{CDCl}_{3}\right)$

$\delta 211.2,151.9,150.0,124.1,51.1,45.8,43.8,41.4,31.7,29.8,29.6,25.2,22.8$, 14.0.

IR $\quad$ Alpha-Platinum ATR, Bruker, diamond crystal

$v=3024,2953,2930,1708,1597,1415 \mathrm{~cm}^{-1}$.

HRMS ESI-TOF

$(\mathrm{M}+\mathrm{H})^{+}$Calcd for $\mathrm{C}_{16} \mathrm{H}_{24} \mathrm{NO} 246.1853$; Found 246.1863. 

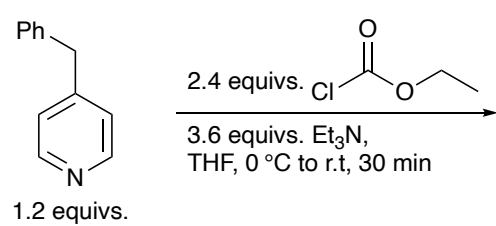
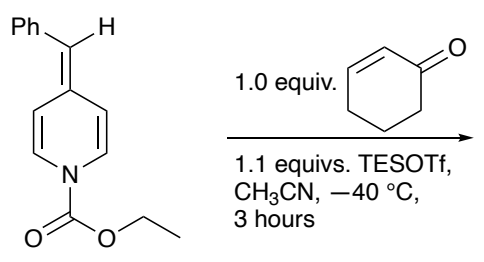
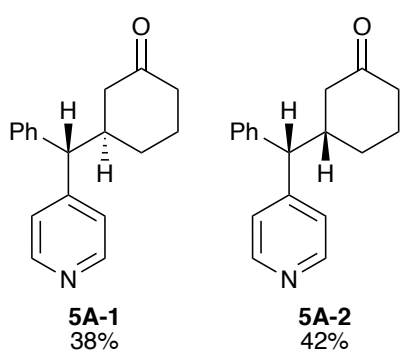

4-Benzyl-pyridine ( $0.19 \mathrm{~mL}, 1.2$ equivs., $1.2 \mathrm{mmol})$ was converted to the corresponding ADHP following general procedure $\mathbf{A}$. Final product 5A was synthesized using general procedure $\mathbf{B}$ with 2-cyclohexen-1-one $(0.1 \mathrm{~mL}, 1.0$ equiv. $1.0 \mathrm{mmol})$ as the electrophile. The mixture of diastereomers was separated by flash column chromatography using MeCN/DCM to afford $100 \mathrm{mg}$ of the anti-diastereomer (5A-1) as white solid and $112 \mathrm{mg}$ of the syn-diastereomer (5A-2) as colorless oil, in $80 \%$ combined yield (d.r. 1.1).

The relative configuration of diastereomers $5 A$ and $5 A^{\prime}$ was assigned based on 2D-NMR studies (page 20 and 21).

Data for the anti-diastereomer (5A-1):

melting point: $156.3-157.6^{\circ} \mathrm{C}$

Chromatography: $10 \%$ MeCN in DCM $\left(R_{f}=0.38\right)$

1 NMR $\quad\left(400 \mathrm{MHz}, \mathrm{CDCl}_{3}\right)$

$\delta 8.70-8.40(\mathrm{~m}, 2 \mathrm{H}), 7.31-7.25(\mathrm{~m}, 2 \mathrm{H}), 7.24-7.17(\mathrm{~m}, 5 \mathrm{H}), 3.61(\mathrm{~d}, J=10.7$

$\mathrm{Hz}, 1 \mathrm{H}), 2.70-2.59(\mathrm{~m}, 1 \mathrm{H}), 2.41-2.22(\mathrm{~m}, 3 \mathrm{H}), 2.07-2.03(\mathrm{~m}, 1 \mathrm{H}), 2.03-1.92$

$(\mathrm{m}, 1 \mathrm{H}), 1.82-1.78(\mathrm{~m}, 1 \mathrm{H}), 1.71-1.60(\mathrm{~m}, 1 \mathrm{H}), 1.39-1.25(\mathrm{~m}, 1 \mathrm{H})$.

${ }^{13} \mathrm{C} \mathrm{NMR} \quad\left(100 \mathrm{MHz}, \mathrm{CDCl}_{3}\right)$

$\delta 210.8,152.2,150.3,140.9,129.2,128.0,127.3,123.5,58.4,46.6,42.0,41.4$, $30.2,24.9$.

Data for the syn-diastereomer (5A-2):

Chromatography: $10 \% \mathrm{MeCN}$ in DCM $\left(R_{\mathrm{f}}=0.28\right)$

1H NMR $\quad\left(400 \mathrm{MHz}, \mathrm{CDCl}_{3}\right)$

$\delta 8.49-8.47(\mathrm{~m}, 2 \mathrm{H}), 7.34-7.28(\mathrm{~m}, 2 \mathrm{H}), 7.26-7.23(\mathrm{~m}, 3 \mathrm{H}), 7.17(\mathrm{~d}, J=5.5$

$\mathrm{Hz}, 2 \mathrm{H}), 3.62(\mathrm{~d}, J=10.7 \mathrm{~Hz}, 1 \mathrm{H}), 2.71-2.60(\mathrm{~m}, 1 \mathrm{H}), 2.41-2.23(\mathrm{~m}, 3 \mathrm{H}), 2.07$

$-1.95(\mathrm{~m}, 2 \mathrm{H}), 1.84-1.80(\mathrm{~m}, 1 \mathrm{H}), 1.70-1.58(\mathrm{~m}, 1 \mathrm{H}), 1.39-1.28(\mathrm{~m}, 1 \mathrm{H})$.

${ }^{13} \mathbf{C}$ NMR $\quad\left(100 \mathrm{MHz}, \mathrm{CDCl}_{3}\right)$

$\delta 210.7,151.5,150.3,141.6,129.1,128.0,127.3,123.4,58.2,46.8,42.0,41.4$, 29.9, 24.9.

IR Alpha-Platinum ATR, Bruker, diamond crystal

$v=3022,2964,2931,1701,1593,1415 \mathrm{~cm}^{-1}$.

HRMS ESI-TOF

$(\mathrm{M}+\mathrm{H})^{+}$Calcd for $\mathrm{C}_{18} \mathrm{H}_{20} \mathrm{NO} 266.1540$; Found 266.1547. 
Anti-diastereomer (5A-1):

$\operatorname{COSY}\left(600 \mathrm{MHz}, \mathrm{C}_{6} \mathrm{H}_{6}\right.$ and $\left.\mathrm{CDCl}_{3}\right)$ :

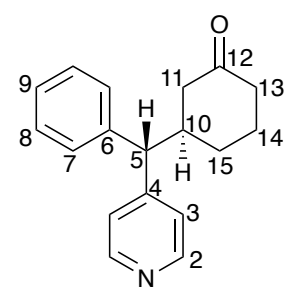

\begin{tabular}{|c|c|c|}
\hline Proton No. & ${ }^{1} \mathrm{H} \delta(\mathrm{ppm})($ mult; $J(\mathrm{~Hz}))$ & COSY Correlations \\
\hline $\mathrm{H}-2$ & $8.41-8.39(\mathrm{~m})$ & $\mathrm{H}-3$ \\
\hline $\mathrm{H}-3$ & $6.91(\mathrm{~d}, J=5.0 \mathrm{~Hz})$ & $\mathrm{H}-2$ \\
\hline $\mathrm{H}-5$ & $3.28(\mathrm{~d}, J=10.8 \mathrm{~Hz})$ & $\mathrm{H}-10$ \\
\hline $\mathrm{H}-7$ & $7.02-6.99(\mathrm{~m})$ & $\mathrm{H}-8$ \\
\hline $\mathrm{H}-8$ & $7.17-7.14(\mathrm{~m})$ & $\mathrm{H}-8, \mathrm{H}-9$ \\
\hline $\mathrm{H}-9$ & $7.10-7.06(\mathrm{~m})$ & $\mathrm{H}-5 ; \mathrm{H}-11 ; \mathrm{H}-15$ \\
\hline $\mathrm{H}-10$ & $2.38-2.29(\mathrm{~m})$ & $\mathrm{H}-10 ; \mathrm{H}-11 \mathrm{~b}$ \\
\hline $\mathrm{H}-11 \mathrm{a}$ & $1.68-1.63(\mathrm{~m})$ & $\mathrm{H}-10 ; \mathrm{H}-11 \mathrm{a}$ \\
\hline $\mathrm{H}-11 \mathrm{~b}$ & $2.20-2.17(\mathrm{~m})$ & $\mathrm{H}-13 \mathrm{~b} ; \mathrm{H}-14$ \\
\hline $\mathrm{H}-13 \mathrm{a}$ & $2.03-1.95(\mathrm{dt}, J=13.4,6.2 \mathrm{~Hz})$ & $\mathrm{H}-13 \mathrm{a} ; \mathrm{H}-14$ \\
\hline $\mathrm{H}-13 \mathrm{~b}$ & $2.26-2.01(\mathrm{~m})$ & $\mathrm{H}-13 ; \mathrm{H}-14 \mathrm{~b} ; \mathrm{H}-15$ \\
\hline $\mathrm{H}-14 \mathrm{a}$ & $1.42-1.33(\mathrm{~m})$ & $\mathrm{H}-13 ; \mathrm{H}-14 \mathrm{a} ; \mathrm{H}-15$ \\
\hline $\mathrm{H}-14 \mathrm{~b}$ & $1.75-1.69(\mathrm{~m})$ & $\mathrm{H}-10 ; \mathrm{H}-15 \mathrm{~b}$ \\
\hline $\mathrm{H}-15 \mathrm{a}$ & $0.99-0.95(\mathrm{~m})$ & $\mathrm{H}-10 ; \mathrm{H}-15 \mathrm{a}$ \\
\hline $\mathrm{H}-15 \mathrm{~b}$ & $1.52-1.46(\mathrm{~m})$ & \\
\hline
\end{tabular}

$\operatorname{NOESY}\left(600 \mathrm{MHz}, \mathrm{C}_{6} \mathrm{H}_{6}\right.$ and $\left.\mathrm{CDCl}_{3}\right)$ :

\begin{tabular}{|c|c|c|}
\hline Proton No. & ${ }^{1} \mathrm{H} \delta(\mathrm{ppm})(\mathrm{mult} ; J(\mathrm{~Hz}))$ & NOESY Correlations \\
\hline $\mathrm{H}-2$ & $8.41-8.39(\mathrm{~m})$ & - \\
\hline $\mathrm{H}-3$ & $6.91(\mathrm{~d}, J=5.0 \mathrm{~Hz})$ & $\mathrm{H}-5 ; \mathrm{H}-10 ; \mathrm{H}-15$ \\
\hline $\mathrm{H}-5$ & $3.28(\mathrm{~d}, J=10.8 \mathrm{~Hz})$ & $\mathrm{H}-3 ; \mathrm{H}-7 ; \mathrm{H}-10 ; \mathrm{H}-11 ; \mathrm{H}-15$ \\
\hline $\mathrm{H}-7$ & $7.02-6.99(\mathrm{~m})$ & - \\
\hline $\mathrm{H}-8$ & $7.17-7.14(\mathrm{~m})$ & - \\
\hline $\mathrm{H}-9$ & $7.10-7.06(\mathrm{~m})$ & $\mathrm{H}-5 ; \mathrm{H}-10 ; \mathrm{H}-11$ \\
\hline $\mathrm{H}-10$ & $2.38-2.29(\mathrm{~m})$ & $\mathrm{H}-7$ \\
\hline $\mathrm{H}-11 \mathrm{a}$ & $1.68-1.63(\mathrm{~m})$ & $\mathrm{H}-5 ; \mathrm{H}-7$ \\
\hline $\mathrm{H}-11 \mathrm{~b}$ & $2.20-2.17(\mathrm{~m})$ & - \\
\hline $\mathrm{H}-13 \mathrm{a}$ & $2.03-1.95(\mathrm{dt}, J=13.4,6.2 \mathrm{~Hz})$ & - \\
\hline $\mathrm{H}-13 \mathrm{~b}$ & $2.26-2.01(\mathrm{~m})$ & - \\
\hline $\mathrm{H}-14 \mathrm{a}$ & $1.42-1.33(\mathrm{~m})$ & - \\
\hline $\mathrm{H}-14 \mathrm{~b}$ & $1.75-1.69(\mathrm{~m})$ & $\mathrm{H}-3 ; \mathrm{H}-5$ \\
\hline $\mathrm{H}-15 a$ & $0.99-0.95(\mathrm{~m})$ & $\mathrm{H}-3 ; \mathrm{H}-5$ \\
\hline $\mathrm{H}-15 \mathrm{~b}$ & $1.52-1.46(\mathrm{~m})$ & \\
\hline
\end{tabular}




\section{Syn-diastereomer (5A-2):}

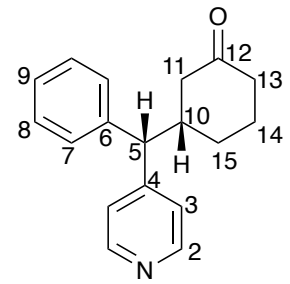

$\operatorname{COSY}\left(600 \mathrm{MHz}, \mathrm{C}_{6} \mathrm{H}_{6}\right.$ and $\left.\mathrm{CDCl}_{3}\right)$ :

\begin{tabular}{|c|c|c|}
\hline Proton No. & ${ }^{1} \mathrm{H} \delta(\mathrm{ppm})(\mathrm{mult} ; J(\mathrm{~Hz}))$ & COSY Correlations \\
\hline $\mathrm{H}-2$ & $8.42-8.41(\mathrm{~m})$ & $\mathrm{H}-3$ \\
\hline $\mathrm{H}-3$ & $6.82-6.80(\mathrm{~m})$ & $\mathrm{H}-2$ \\
\hline $\mathrm{H}-5$ & $3.23-3.20(\mathrm{~m})$ & $\mathrm{H}-10$ \\
\hline $\mathrm{H}-7$ & $7.02-7.00(\mathrm{~m})$ & $\mathrm{H}-8, \mathrm{H}-9$ \\
\hline $\mathrm{H}-8$ & $7.19-7.16(\mathrm{~m})$ & $\mathrm{H}-8$ \\
\hline $\mathrm{H}-9$ & $7.12-7.08(\mathrm{~m})$ & $\mathrm{H}-5 ; \mathrm{H}-11 ; \mathrm{H}-15$ \\
\hline $\mathrm{H}-10$ & $2.32-2.27(\mathrm{~m})$ & $\mathrm{H}-10 ; \mathrm{H}-11 \mathrm{~b}$ \\
\hline $\mathrm{H}-11 \mathrm{a}$ & $1.60-1.55(\mathrm{~m})$ & $\mathrm{H}-13 \mathrm{H}-11 \mathrm{a}$ \\
\hline $\mathrm{H}-11 \mathrm{~b}$ & $2.22-14$ \\
\hline $\mathrm{H}-13 \mathrm{a}$ & $1.96-13(\mathrm{~m})$ & $\mathrm{H}-13 \mathrm{~m} ; \mathrm{H}-14$ \\
\hline $\mathrm{H}-13 \mathrm{~b}$ & $2.24-2.13(\mathrm{~m})$ & $\mathrm{H}-13 ; \mathrm{H}-14 \mathrm{~b} ; \mathrm{H}-15$ \\
\hline $\mathrm{H}-14 \mathrm{a}$ & $1.35-1.26(\mathrm{~m})$ & $\mathrm{H}-13 ; \mathrm{H}-14 \mathrm{a} ; \mathrm{H}-15$ \\
\hline $\mathrm{H}-14 \mathrm{~b}$ & $1.66-1.60(\mathrm{~m})$ & $\mathrm{H}-10 ; \mathrm{H}-15 \mathrm{~b}$ \\
\hline $\mathrm{H}-15 \mathrm{a}$ & $0.95-0.84(\mathrm{~m})$ & $\mathrm{H}-10 ; \mathrm{H}-15 \mathrm{a}$ \\
\hline $\mathrm{H}-15 \mathrm{~b}$ & $1.54-1.47(\mathrm{~m})$ & \\
\hline
\end{tabular}

NOESY $\left(600 \mathrm{MHz}, \mathrm{C}_{6} \mathrm{H}_{6}\right.$ and $\left.\mathrm{CDCl}_{3}\right)$ :

\begin{tabular}{|c|c|c|}
\hline Proton No. & ${ }^{1} \mathrm{H} \delta(\mathrm{ppm})(\mathrm{mult} ; J(\mathrm{~Hz}))$ & NOESY Correlations \\
\hline $\mathrm{H}-2$ & $8.42-8.41(\mathrm{~m})$ & - \\
\hline $\mathrm{H}-3$ & $6.82-6.80(\mathrm{~m})$ & $\mathrm{H}-5 ; \mathrm{H}-10 ; \mathrm{H}-11$ \\
\hline $\mathrm{H}-5$ & $3.23-3.20(\mathrm{~m})$ & $\mathrm{H}-3 ; \mathrm{H}-7 ; \mathrm{H}-10 ; \mathrm{H}-11 ; \mathrm{H}-15$ \\
\hline $\mathrm{H}-7$ & $7.02-7.00(\mathrm{~m})$ & - \\
\hline $\mathrm{H}-8$ & $7.19-7.16(\mathrm{~m})$ & - \\
\hline $\mathrm{H}-9$ & $7.12-7.08(\mathrm{~m})$ & $\mathrm{H}-5 ; \mathrm{H}-10 ; \mathrm{H}-15$ \\
\hline $\mathrm{H}-10$ & $2.32-2.27(\mathrm{~m})$ & $\mathrm{H}-3 ; \mathrm{H}-5-7$ \\
\hline $\mathrm{H}-11 \mathrm{a}$ & $1.60-1.55(\mathrm{~m})$ & $\mathrm{H}-3 ; \mathrm{H}-5$ \\
\hline $\mathrm{H}-11 \mathrm{~b}$ & $2.22-2.13(\mathrm{~m})$ & - \\
\hline $\mathrm{H}-13 a$ & $1.96-1.88(\mathrm{~m})$ & - \\
\hline $\mathrm{H}-13 \mathrm{~b}$ & $2.24-2.13(\mathrm{~m})$ & - \\
\hline $\mathrm{H}-14 \mathrm{a}$ & $1.35-1.26(\mathrm{~m})$ & - \\
\hline $\mathrm{H}-14 \mathrm{~b}$ & $1.66-1.60(\mathrm{~m})$ & $\mathrm{H}-3 ; \mathrm{H}-5$ \\
\hline $\mathrm{H}-15 \mathrm{a}$ & $0.95-0.84(\mathrm{~m})$ & $\mathrm{H}-3 ; \mathrm{H}-5$ \\
\hline $\mathrm{H}-15 \mathrm{~b}$ & $1.54-1.47(\mathrm{~m})$ & \\
\hline
\end{tabular}




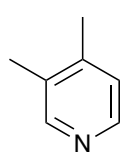

1.2 equivs

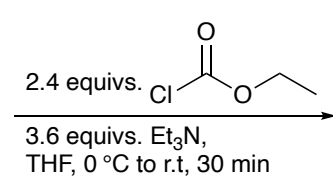

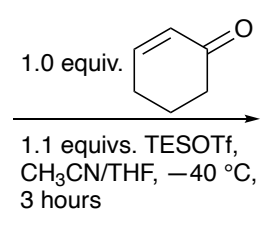

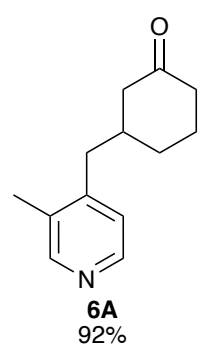

3,4-Lutidine ( $0.14 \mathrm{~mL}, 1.2$ equivs., $1.2 \mathrm{mmol})$ was converted to the corresponding ADHP following general procedure $\mathbf{A}$. Final product $6 \mathrm{~A}$ was synthesized following general procedure B using anhydrous THF $(0.2 \mathrm{M})$ to dissolve the ADHP instead of $\mathrm{CH}_{3} \mathrm{CN}$ due to its poor solubility in $\mathrm{CH}_{3} \mathrm{CN}$, and 2-cyclohexen-1-one $(0.1 \mathrm{~mL}, 1.0$ equiv., $1.0 \mathrm{mmol})$ as the electrophile. The crude product was purified by flash column chromatography using EtOAc/hexanes/ $\mathrm{Et}_{3} \mathrm{~N}$ to afford compound 6A as colorless oil (187 $\mathrm{mg}, 92 \%$ yield).

Chromatography: $70 \%$ EtOAc in hexanes with $2 \% \mathrm{Et}_{3} \mathrm{~N} .\left(\mathrm{R}_{\mathrm{f}}=0.34\right)$

1H NMR $\quad\left(400 \mathrm{MHz}, \mathrm{CDCl}_{3}\right)$

$\delta 8.35(\mathrm{~s}, 1 \mathrm{H}), 8.32(\mathrm{~d}, J=5.0 \mathrm{~Hz}, 1 \mathrm{H}), 6.96(\mathrm{~d}, J=5.0 \mathrm{~Hz}, 1 \mathrm{H}), 2.66-2.55(\mathrm{~m}$, $2 \mathrm{H}), 2.40-2.32(\mathrm{~m}, 2 \mathrm{H}), 2.32-2.23(\mathrm{~m}, 4 \mathrm{H}), 2.14-2.00(\mathrm{~m}, 3 \mathrm{H}), 1.89-1.83(\mathrm{~m}$, $1 \mathrm{H}), 1.68-1.55(\mathrm{~m}, 1 \mathrm{H}), 1.48-1.36(\mathrm{~m}, 1 \mathrm{H})$

${ }^{13} \mathrm{C} \mathrm{NMR} \quad\left(100 \mathrm{MHz}, \mathrm{CDCl}_{3}\right)$

$\delta 210.8,151.3,147.5,146.6,131.8,124.5,48.0,41.4,39.5,39.2,31.2,25.1,16.4$.

IR Alpha-Platinum ATR, Bruker, diamond crystal $v=3022,2932,2869,1706,1594,1449 \mathrm{~cm}^{-1}$.

HRMS ESI-TOF

$(\mathrm{M}+\mathrm{H})^{+}$Calcd for $\mathrm{C}_{13} \mathrm{H}_{18} \mathrm{NO} 204.1383$; Found 204.1391. 


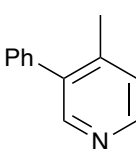

1.2 equivs

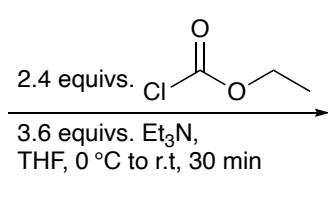

THF, $0^{\circ} \mathrm{C}$ to r.t, $30 \mathrm{~min}$<smiles></smiles><smiles>Cn1ccc(CC2CCCC(=O)C2)c1</smiles>

3-Phenyl-4-methyl-pyridine (203.1 mg, 1.2 equivs., $1.2 \mathrm{mmol}$ ) was converted into the corresponding ADHP following general procedure A. Final product 7A was synthesized following general procedure $\mathbf{B}$ using anhydrous THF $(0.2 \mathrm{M})$ to dissolve the ADHP instead of $\mathrm{CH}_{3} \mathrm{CN}$ due to its poor solubility in $\mathrm{CH}_{3} \mathrm{CN}$, and 2-cyclohexen-1-one $(0.1 \mathrm{~mL}, 1.0$ equiv., $1.0 \mathrm{mmol})$ as the electrophile. The crude product was purified by flash column chromatography using EtOAc/hexanes/Et ${ }_{3} \mathrm{~N}$ to afford compound $7 \mathrm{~A}$ as light-yellow oil ( $227 \mathrm{mg}, 85 \%$ yield).

Chromatography: $60 \%$ EtOAc in hexanes with $2 \% \mathrm{Et}_{3} \mathrm{~N} .\left(\mathrm{R}_{\mathrm{f}}=0.31\right)$

1H NMR $\quad\left(400 \mathrm{MHz}, \mathrm{CDCl}_{3}\right)$

$\delta 8.48(\mathrm{~d}, J=5.1 \mathrm{~Hz}, 1 \mathrm{H}), 8.42(\mathrm{~s}, 1 \mathrm{H}), 7.46-7.36(\mathrm{~m}, 3 \mathrm{H}), 7.27-7.24(\mathrm{~m}, 2 \mathrm{H})$, $7.14(\mathrm{~d}, J=5.1 \mathrm{~Hz}, 1 \mathrm{H}), 2.68(\mathrm{dd}, J=13.5,6.8 \mathrm{~Hz}, 1 \mathrm{H}), 2.62(\mathrm{dd}, J=13.5,6.7$ $\mathrm{Hz}, 1 \mathrm{H}), 2.30-2.22(\mathrm{~m}, 1 \mathrm{H}), 2.21-2.10(\mathrm{~m}, 2 \mathrm{H}), 1.94-1.80(\mathrm{~m}, 3 \mathrm{H}), 1.69-1.65$ $(\mathrm{m}, 1 \mathrm{H}), 1.54-1.42(\mathrm{~m}, 1 \mathrm{H}), 1.21-1.10(\mathrm{~m}, 1 \mathrm{H})$.

${ }^{13} \mathrm{C}$ NMR $\quad\left(100 \mathrm{MHz}, \mathrm{CDCl}_{3}\right)$

$\delta 210.8,150.7,148.7,146.0 .138 .1,137.8,129.5,128.6,127.9,124.5,47.8,41.3$, $39.8,39.1,30.9,25.0$.

IR Alpha-Platinum ATR, Bruker, diamond crystal $v=3023,2934,2866,1706,1588,1445 \mathrm{~cm}^{-1}$.

HRMS ESI-TOF

$(\mathrm{M}+\mathrm{H})^{+}$Calcd for $\mathrm{C}_{18} \mathrm{H}_{20} \mathrm{NO} 266.1540$; Found 266.1542. 


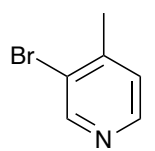

1.2 equivs.

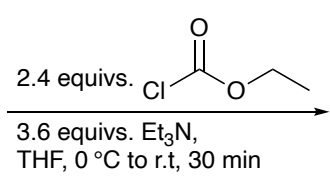

THF, $0{ }^{\circ} \mathrm{C}$ to r.t, $30 \mathrm{~min}$<smiles></smiles>

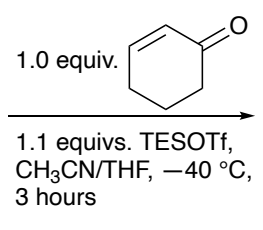

3-Bromo-4-methylpyridine $(0.14 \mathrm{~mL}, 1.2$ equivs., $1.2 \mathrm{mmol})$ was converted to the corresponding ADHP following general procedure $\mathbf{A}$. Final product $\mathbf{8 A}$ was synthesized using general procedure B but using anhydrous THF $(0.2 \mathrm{M})$ to dissolve the ADHP instead of $\mathrm{CH}_{3} \mathrm{CN}$ due to its poor solubility in $\mathrm{CH}_{3} \mathrm{CN}$, and 2-cyclohexen-1-one $(0.1 \mathrm{~mL}, 1.0$ equiv., $1.0 \mathrm{mmol})$ as the electrophile. The crude product was purified by flash column chromatography using EtOAc/hexanes to afford compound 8A as light-yellow oil (205 mg, 76\% yield).

Chromatography: $70 \%$ EtOAc in hexanes. $\left(R_{f}=0.42\right)$

1․ NMR $\quad\left(400 \mathrm{MHz}, \mathrm{CDCl}_{3}\right)$

$\delta 8.67(\mathrm{~s}, 1 \mathrm{H}), 8.41(\mathrm{~d}, J=4.9 \mathrm{~Hz}, 1 \mathrm{H}), 7.09(\mathrm{~d}, J=4.9 \mathrm{~Hz}, 1 \mathrm{H}), 2.79(\mathrm{dd}, J=13.2$, $6.8 \mathrm{~Hz}, 1 \mathrm{H}), 2.71(\mathrm{dd}, J=13.2,6.6 \mathrm{~Hz}, 1 \mathrm{H}), 2.41-2.34(\mathrm{~m}, 2 \mathrm{H}), 2.33-2.24(\mathrm{~m}$, $1 \mathrm{H}), 2.23-2.02(\mathrm{~m}, 3 \mathrm{H}), 1.92-1.84(\mathrm{~m}, 1 \mathrm{H}), 1.70-1.57(\mathrm{~m}, 1 \mathrm{H}), 1.52-1.41(\mathrm{~m}$, $1 \mathrm{H})$.

${ }^{13} \mathrm{C} \mathrm{NMR} \quad\left(100 \mathrm{MHz}, \mathrm{CDCl}_{3}\right)$

$\delta$ 210.6, 152.4, 148.2, 147.8, 126.1, 123.5, 47.6, 42.1, 41.4, 38.8, 31.0, 25.0.

IR Alpha-Platinum ATR, Bruker, diamond crystal

$v=3042,2934,2868,1706,1582,1398,511 \mathrm{~cm}^{-1}$.

HRMS ESI-TOF

$(\mathrm{M}+\mathrm{H})^{+}$Calcd for $\mathrm{C}_{12} \mathrm{H}_{15} \mathrm{BrNO} 268.0332$; Found 268.0347. 


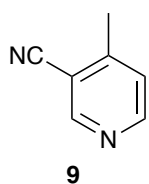

1.2 equivs

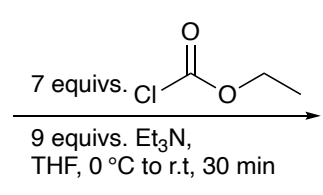

$\mathrm{THF}, 0^{\circ} \mathrm{C}$ to r.t, $30 \mathrm{~min}$<smiles></smiles>

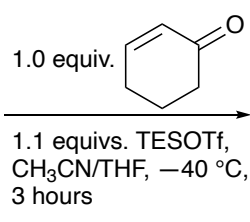

To a dry round-bottomed flask containing anhydrous THF $(0.1 \mathrm{M})$ were added compound 9 (71 mg, 1.2 equivs., $0.6 \mathrm{mmol})$ and triethylamine $(0.75 \mathrm{~mL}, 9.0$ equivs., $5.4 \mathrm{mmol})$ at ambient temperature. The resulting solution was cooled to $0{ }^{\circ} \mathrm{C}$ and ethyl chloroformate $(0.4 \mathrm{~mL}, 7.0$ equivs., $4.2 \mathrm{mmol}$ ) was added dropwise. The resulting mixture was stirred at ambient temperature for 8 hours and then concentrated using a rotary evaporator. The residue was dissolved in diethyl ether, triturated for 2-3 minutes and filtered through a cotton plug to remove the precipitated triethyl ammonium chloride. The filtrate was concentrated using a rotary evaporator to give the corresponding ADHP as pale yellow oil, which was used for next step without further purification.

Final product 9A was synthesized following general procedure B but using anhydrous THF (0.2 M) to dissolve the ADHP instead of $\mathrm{CH}_{3} \mathrm{CN}$ due to its poor solubility in $\mathrm{CH}_{3} \mathrm{CN}$, and 2cyclohexen-1-one $(0.05 \mathrm{~mL}, 1.0$ equiv., $0.5 \mathrm{mmol})$ as the electrophile. The crude product was purified by flash column chromatography using EtOAc/hexanes/Et ${ }_{3} \mathrm{~N}$ to afford compound $\mathbf{9 A}$ as a light-yellow oil (38 mg, 35\% yield).

Melting point: $60.7-61.9^{\circ} \mathrm{C}$

Chromatography: $50 \%$ EtOAc in hexanes with $2 \% \mathrm{Et}_{3} \mathrm{~N} .\left(\mathrm{R}_{\mathrm{f}}=0.35\right)$

1 NMR $\quad\left(400 \mathrm{MHz}, \mathrm{CDCl}_{3}\right)$

$\delta 8.84(\mathrm{~s}, 1 \mathrm{H}), 8.70(\mathrm{~d}, J=5.1 \mathrm{~Hz}, 1 \mathrm{H}), 7.23(\mathrm{~d}, J=5.1 \mathrm{~Hz}, 1 \mathrm{H}), 2.93(\mathrm{dd}, J=13.5$, $6.5 \mathrm{~Hz}, 1 \mathrm{H}), 2.82(\mathrm{dd}, J=13.5,6.9 \mathrm{~Hz}, 1 \mathrm{H}), 2.43-2.25(\mathrm{~m}, 3 \mathrm{H}), 2.25-2.06(\mathrm{~m}$, $3 \mathrm{H}), 1.95-1.85(\mathrm{~m}, 1 \mathrm{H}), 1.72-1.60(\mathrm{~m}, 1 \mathrm{H}), 1.57-1.46(\mathrm{~m}, 1 \mathrm{H})$.

${ }^{13} \mathbf{C ~ N M R} \quad\left(100 \mathrm{MHz}, \mathrm{CDCl}_{3}\right)$

$\delta 209.7,153.2,152.7,152.1,124.5,115.9,110.9,47.3,41.1,40.7,39.8,30.9,24.8$.

IR Alpha-Platinum ATR, Bruker, diamond crystal $v=3022,2958,2928,2226,1700,1588,1416 \mathrm{~cm}^{-1}$.

HRMS ESI-TOF

$(\mathrm{M}+\mathrm{H})^{+}$Calcd for $\mathrm{C}_{13} \mathrm{H}_{15} \mathrm{~N}_{2} \mathrm{O} 215.1179$; Found 215.1186. 
<smiles>Cc1ccncc1C(=O)NC(C)(C)C</smiles>

1.2 equivs.

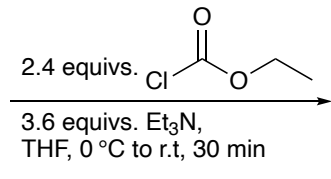

THF, $0^{\circ} \mathrm{C}$ to r.t, $30 \mathrm{~min}$<smiles></smiles>
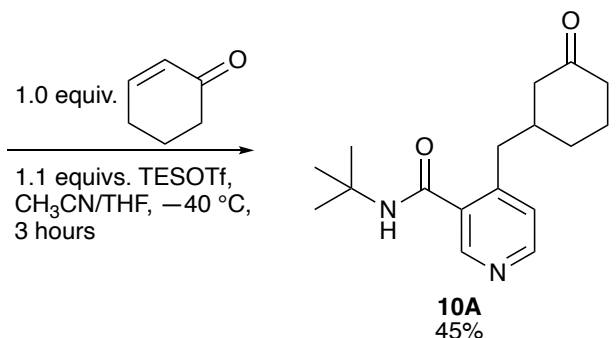

Pyridine 10 (115.4 mg, 1.2 equivs., $0.6 \mathrm{mmol}$ ) was converted to the corresponding ADHP following general procedure $\mathrm{A}$. Final product 10A was synthesized following general procedure B but using anhydrous THF $(0.2 \mathrm{M})$ to dissolve the ADHP instead of $\mathrm{CH}_{3} \mathrm{CN}$ due to its poor solubility in $\mathrm{CH}_{3} \mathrm{CN}$, and 2-cyclohexen-1-one $(0.05 \mathrm{~mL}, 1.0$ equiv., $0.5 \mathrm{mmol})$ as the electrophile. The crude product was purified by flash column chromatography using EtOAc/hexanes/Et ${ }_{3} \mathrm{~N}$ to afford compound $10 \mathrm{~A}$ as white solid (154 $\mathrm{mg}, 83 \%$ yield).

Melting point: $96.2-96.7^{\circ} \mathrm{C}$

Chromatography: $50 \%$ EtOAc in hexanes with $2 \% \mathrm{Et}_{3} \mathrm{~N} .\left(\mathrm{R}_{\mathrm{f}}=0.20\right)$

1H NMR $\quad\left(400 \mathrm{MHz}, \mathrm{CDCl}_{3}\right)$

$\delta 8.51(\mathrm{~s}, 1 \mathrm{H}), 8.47(\mathrm{~d}, J=5.0 \mathrm{~Hz}, 1 \mathrm{H}), 7.08(\mathrm{~d}, J=5.0 \mathrm{~Hz}, 1 \mathrm{H}), 5.84(\mathrm{~s}, 1 \mathrm{H}), 2.95$ (dd, $J=13.2,6.2 \mathrm{~Hz}, 1 \mathrm{H}), 2.73(\mathrm{dd}, J=13.2,7.2 \mathrm{~Hz}, 1 \mathrm{H}), 2.36-2.21(\mathrm{~m}, 3 \mathrm{H})$, $2.17-2.02(\mathrm{~m}, 3 \mathrm{H}), 1.90-1.81(\mathrm{~m}, 1 \mathrm{H}), 1.67-1.55(\mathrm{~m}, 1 \mathrm{H}), 1.50-1.38(\mathrm{~m}, 10 \mathrm{H})$.

${ }^{13} \mathrm{C} \mathrm{NMR} \quad\left(100 \mathrm{MHz}, \mathrm{CDCl}_{3}\right)$

$\delta 210.9,167.1,150.6,147.6,147.4,133.8,52.4,47.7,41.4,40.1,39.2,31.4,28.9$, 25.1.

IR Alpha-Platinum ATR, Bruker, diamond crystal $v=3363,3017,2956,2852,1711,1650,1463 \mathrm{~cm}^{-1}$.

HRMS ESI-TOF

$(\mathrm{M}+\mathrm{H})^{+}$Calcd for $\mathrm{C}_{17} \mathrm{H}_{25} \mathrm{~N}_{2} \mathrm{O}_{2} 289.1911$; Found 289.1924. 
<smiles>CCOC(=O)c1cnccc1C</smiles>

1.2 equivs.

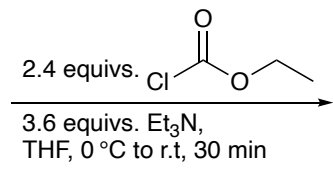

THF, $0^{\circ} \mathrm{C}$ to r.t, $30 \mathrm{~min}$<smiles></smiles>

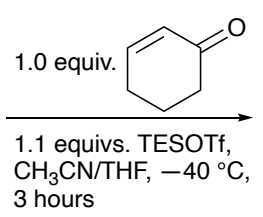<smiles>CCOC(=O)c1cnccc1CC1CCCC(=O)C1</smiles>

Ethyl 4-methylnicotinate 11 (99 mg, 1.2 equivs., $0.6 \mathrm{mmol}$ ) was converted to the corresponding ADHP following general procedure $\mathbf{A}$. Final product $11 \mathrm{~A}$ was synthesized following general procedure $\mathbf{B}$ but using anhydrous THF $(0.2 \mathrm{M})$ to dissolve the ADHP instead of $\mathrm{CH}_{3} \mathrm{CN}$ due to its poor solubility in $\mathrm{CH}_{3} \mathrm{CN}$, and 2-cyclohexen-1-one $(0.05 \mathrm{~mL}, 1.0$ equiv., 0.5 $\mathrm{mmol}$ ) as the electrophile. The crude product was purified by flash column chromatography using EtOAc/hexanes/Et ${ }_{3} \mathrm{~N}$ to afford compound $11 \mathrm{~A}$ as light-yellow oil (71 mg, $54 \%$ yield).

Chromatography: $50 \%$ EtOAc in hexanes with $2 \% \mathrm{Et}_{3} \mathrm{~N} .\left(\mathrm{R}_{\mathrm{f}}=0.38\right)$

1H NMR $\quad\left(400 \mathrm{MHz}, \mathrm{CDCl}_{3}\right)$

$\delta 9.10(\mathrm{~s}, 1 \mathrm{H}), 8.62-8.59(\mathrm{~m}, 1 \mathrm{H}), 7.11(\mathrm{~d}, J=4.8 \mathrm{~Hz}, 1 \mathrm{H}), 4.38(\mathrm{q}, J=7.1 \mathrm{~Hz}$, $2 \mathrm{H}), 3.10(\mathrm{dd}, J=12.7,6.0 \mathrm{~Hz}, 1 \mathrm{H}), 2.97(\mathrm{dd}, J=12.7,6.3 \mathrm{~Hz}, 1 \mathrm{H}), 2.38-2.33$ $(\mathrm{m}, 2 \mathrm{H}), 2.32-2.23(\mathrm{~m} .1 \mathrm{H}), 2.15-2.10(\mathrm{~m}, 2 \mathrm{H}), 2.08-2.01(\mathrm{~m}, 1 \mathrm{H}), 1.89-1.85$ $(\mathrm{m}, 1 \mathrm{H}), 1.68-1.56(\mathrm{~m}, 1 \mathrm{H}), 1.48-1.43(\mathrm{~m}, 1 \mathrm{H}), 1.41(\mathrm{t}, J=7.1 \mathrm{~Hz}, 3 \mathrm{H})$.

${ }^{13} \mathbf{C ~ N M R} \quad\left(100 \mathrm{MHz}, \mathrm{CDCl}_{3}\right)$

$\delta 210.9,166.1,152.3,152.2,150.6,126.4,126.2,61.5,47.8,41.5,40.3,40.2,31.3$, $25.1,14.4$.

IR Alpha-Platinum ATR, Bruker, diamond crystal $v=3043,2979,2935,1706,1590,1418,1274 \mathrm{~cm}^{-1}$.

HRMS ESI-TOF

$(\mathrm{M}+\mathrm{H})^{+}$Calcd for $\mathrm{C}_{15} \mathrm{H}_{20} \mathrm{NO}_{3} 262.1438$; Found 262.1453. 
<smiles>CON(C)C(=O)c1cnccc1C</smiles>

1.2 equivs.

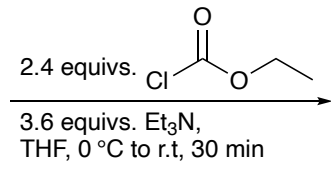

THF, $0^{\circ} \mathrm{C}$ to r.t, $30 \mathrm{~min}$<smiles></smiles>

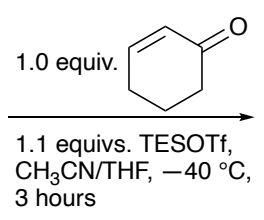<smiles>CON(C)C(=O)c1cnccc1CC1CCCC(=O)C1</smiles>

Pyridine 12 (87 mg, 1.2 equivs., $0.48 \mathrm{mmol}$ ) was converted to the corresponding ADHP following general procedure $\mathrm{A}$. Final product 12A was synthesized following general procedure B but using anhydrous THF $(0.2 \mathrm{M})$ to dissolve the ADHP instead of $\mathrm{CH}_{3} \mathrm{CN}$ due to its poor solubility in $\mathrm{CH}_{3} \mathrm{CN}$, and 2-cyclohexen-1-one ( $0.04 \mathrm{~mL}, 1.0$ equiv., $\left.0.4 \mathrm{mmol}\right)$ as the electrophile. The crude product was purified by flash column chromatography using EtOAc/hexanes/Et ${ }_{3} \mathrm{~N}$ to afford compound product 12A as light-yellow oil (11 mg, 10\% yield).

Chromatography: $40 \%$ EtOAc in hexanes with $2 \% \mathrm{Et}_{3} \mathrm{~N} .\left(\mathrm{R}_{\mathrm{f}}=0.19\right)$

1H NMR $\quad\left(400 \mathrm{MHz}, \mathrm{CDCl}_{3}\right)$

$\delta 8.56-8.52(\mathrm{~m}, 2 \mathrm{H}), 7.17(\mathrm{~d}, J=5.0 \mathrm{~Hz}, 1 \mathrm{H}), 3.50(\mathrm{~s}, 3 \mathrm{H}), 3.35(\mathrm{~s}, 3 \mathrm{H}), 2.72(\mathrm{dd}$, $J=13.6,7.1 \mathrm{~Hz}, 1 \mathrm{H}), 2.66(\mathrm{dd}, J=13.6,6.7 \mathrm{~Hz}, 1 \mathrm{H}), 2.38-2.34(\mathrm{~m}, 2 \mathrm{H}), 2.31-$ $2.21(\mathrm{~m}, 1 \mathrm{H}), 2.20-2.02(\mathrm{~m}, 3 \mathrm{H}), 1.88-1.81(\mathrm{~m}, 1 \mathrm{H}), 1.68-1.55(\mathrm{~m}, 1 \mathrm{H}), 1.44$ $-1.34(\mathrm{~m}, 1 \mathrm{H})$.

${ }^{13} \mathrm{C} \mathrm{NMR} \quad\left(100 \mathrm{MHz}, \mathrm{CDCl}_{3}\right)$ $\delta 210.7,168.6,149.9,147.2,147.0,131.8,124.9,61.5,47.9,41.4,39.6,39.5,33.0$, $31.1,25.1$.

IR Alpha-Platinum ATR, Bruker, diamond crystal $v=3042,2935,2868,1708,1646,1559,1448,1380,1225 \mathrm{~cm}^{-1}$.

HRMS ESI-TOF

$(\mathrm{M}+\mathrm{H})^{+}$Calcd for $\mathrm{C}_{15} \mathrm{H}_{21} \mathrm{~N}_{2} \mathrm{O}_{3} 277.1547$; Found 277.1554. 
<smiles>CCOC(=O)Nc1cnccc1C</smiles>

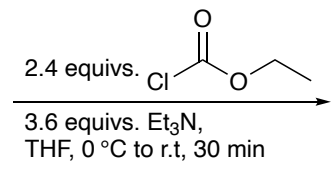
13
1.2
equivs.<smiles></smiles>

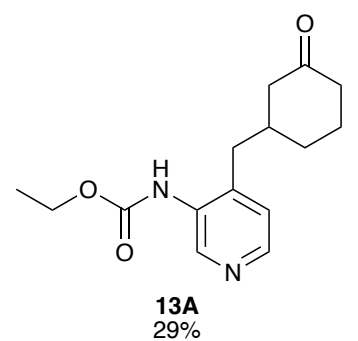

Pyridine 13 (216 mg, 1.2 equivs., $1.2 \mathrm{mmol}$ ) was converted to the corresponding ADHP following general procedure $\mathrm{A}$. Final product 13A was synthesized following general procedure B but using anhydrous THF $(0.2 \mathrm{M})$ to dissolve the ADHP instead of $\mathrm{CH}_{3} \mathrm{CN}$ due to its poor solubility in $\mathrm{CH}_{3} \mathrm{CN}$, and 2-cyclohexen-1-one $(0.1 \mathrm{~mL}, 1.0$ equiv., $1.0 \mathrm{mmol})$ as the electrophile. The crude product was purified by flash column chromatography using EtOAc/hexanes/Et ${ }_{3} \mathrm{~N}$ to afford compound $13 \mathrm{~A}$ as light-yellow oil $(81 \mathrm{mg}, 29 \%$ yield).

Chromatography: $60 \%$ EtOAc in hexanes with $2 \% \mathrm{Et}_{3} \mathrm{~N} .\left(\mathrm{R}_{\mathrm{f}}=0.37\right)$

1H NMR $\quad\left(400 \mathrm{MHz}, \mathrm{CDCl}_{3}\right)$

$\delta 8.81(\mathrm{~s}, 1 \mathrm{H}), 8.33(\mathrm{~d}, J=5.0 \mathrm{~Hz}, 1 \mathrm{H}), 7.06(\mathrm{~d}, J=5.0 \mathrm{~Hz}, 1 \mathrm{H}), 6.47(\mathrm{~s}, 1 \mathrm{H}), 4.24$ (q, $J=7.1 \mathrm{~Hz}, 2 \mathrm{H}), 2.61(\mathrm{~d}, J=6.9 \mathrm{~Hz}, 2 \mathrm{H}), 2.42-2.36(\mathrm{~m}, 2 \mathrm{H}), 2.33-2.24(\mathrm{~m}$, $1 \mathrm{H}), 2.17-2.00(\mathrm{~m}, 3 \mathrm{H}), 1.90-1.83(\mathrm{~m}, 1 \mathrm{H}), 1.74-1.60(\mathrm{~m}, 1 \mathrm{H}), 1.49-1.40(\mathrm{~m}$. $1 \mathrm{H}), 1.32(\mathrm{t}, J=7.1 \mathrm{~Hz}, 3 \mathrm{H})$.

${ }^{13} \mathrm{C} \mathrm{NMR} \quad\left(100 \mathrm{MHz}, \mathrm{CDCl}_{3}\right)$

$\delta 210.9,154.4,146.4,146.4,141.0,132.7,124.8,62.0,47.7,41.4,39.2,37.2,31.0$, 25.0, 14.7 .

IR Alpha-Platinum ATR, Bruker, diamond crystal $v=3299,3039,2928,2867,1739,1708,1600,1416 \mathrm{~cm}^{-1}$.

HRMS ESI-TOF

$(\mathrm{M}+\mathrm{H})^{+}$Calcd for $\mathrm{C}_{15} \mathrm{H}_{21} \mathrm{~N}_{2} \mathrm{O}_{3}$ 277.1547; Found 277.1551. 

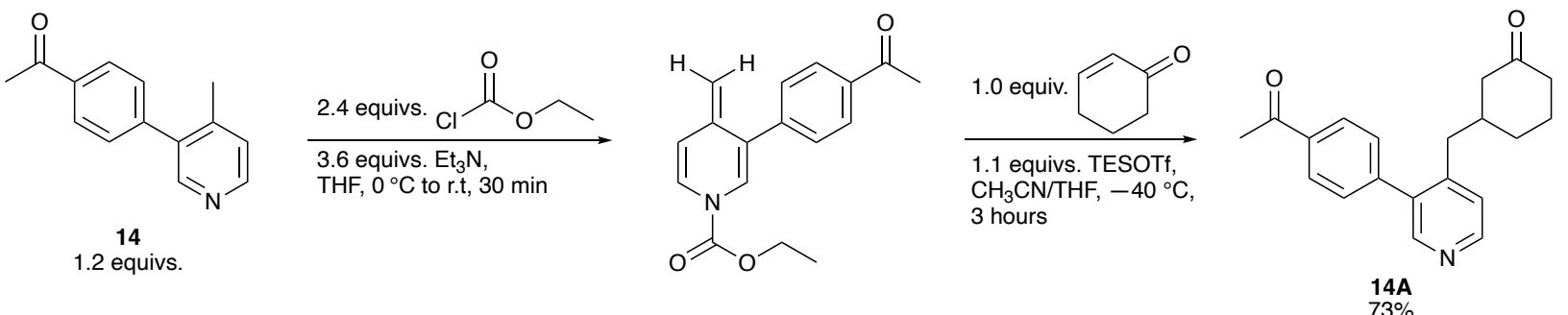

Pyridine 14 (254 mg, 1.2 equivs., $1.2 \mathrm{mmol}$ ) was converted to the corresponding ADHP following general procedure $A$. Final product 14A was prepared following general procedure $B$ but using anhydrous THF ( $0.2 \mathrm{M})$ to dissolve the ADHP instead of $\mathrm{CH}_{3} \mathrm{CN}$ due to its poor solubility in $\mathrm{CH}_{3} \mathrm{CN}$, and 2-cyclohexe-1-none $(0.1 \mathrm{~mL}, 1.0$ equiv., $1.0 \mathrm{mmol})$ was used as the electrophile. The crude product was purified by flash column chromatography using EtOAc/hexanes/Et ${ }_{3} \mathrm{~N}$ to afford compound 14A as white solid (225 mg, 73\% yield).

Melting point: $65.9-67.0^{\circ} \mathrm{C}$

Chromatography: $70 \%$ EtOAc in hexanes with $2 \% \mathrm{Et}_{3} \mathrm{~N} .\left(\mathrm{R}_{\mathrm{f}}=0.25\right)$

1H NMR $\quad\left(400 \mathrm{MHz}, \mathrm{CDCl}_{3}\right)$

$\delta 8.51(\mathrm{~d}, J=5.1 \mathrm{~Hz}, 1 \mathrm{H}), 8.41(\mathrm{~s}, 1 \mathrm{H}), 8.02(\mathrm{~d}, J=8.4 \mathrm{~Hz}, 2 \mathrm{H}), 7.37(\mathrm{~d}, J=8.4$ $\mathrm{Hz}, 2 \mathrm{H}), 7.16(\mathrm{~d}, J=5.1 \mathrm{~Hz}, 1 \mathrm{H}), 2.71-2.58(\mathrm{~m}, 5 \mathrm{H}), 2.30-2.23(\mathrm{~m}, 1 \mathrm{H}), 2.20-$ $2.09(\mathrm{~m}, 2 \mathrm{H}), 1.94-1.78(\mathrm{~m}, 3 \mathrm{H}), 1.69-1.63(\mathrm{~m}, 1 \mathrm{H}), 1.53-1.41(\mathrm{~m}, 1 \mathrm{H}), 1.20$ $-1.09(\mathrm{~m}, 1 \mathrm{H})$.

${ }^{13} \mathrm{C} \mathrm{NMR} \quad\left(100 \mathrm{MHz}, \mathrm{CDCl}_{3}\right)$

$\delta 210.5,197.6,150.3,149.2,145.9,142.8,137.1,136.5,129.8,128.7,124.6,47.7$, $41.2,39.8,39.1,30.9,26.8,24.9$.

IR Alpha-Platinum ATR, Bruker, diamond crystal $v=3042,2935,2868,1708,1681,1606,1270 \mathrm{~cm}^{-1}$.

HRMS ESI-TOF

$(\mathrm{M}+\mathrm{H})^{+}$Calcd for $\mathrm{C}_{20} \mathrm{H}_{22} \mathrm{NO}_{2}$ 308.1645; Found 308.1647. 

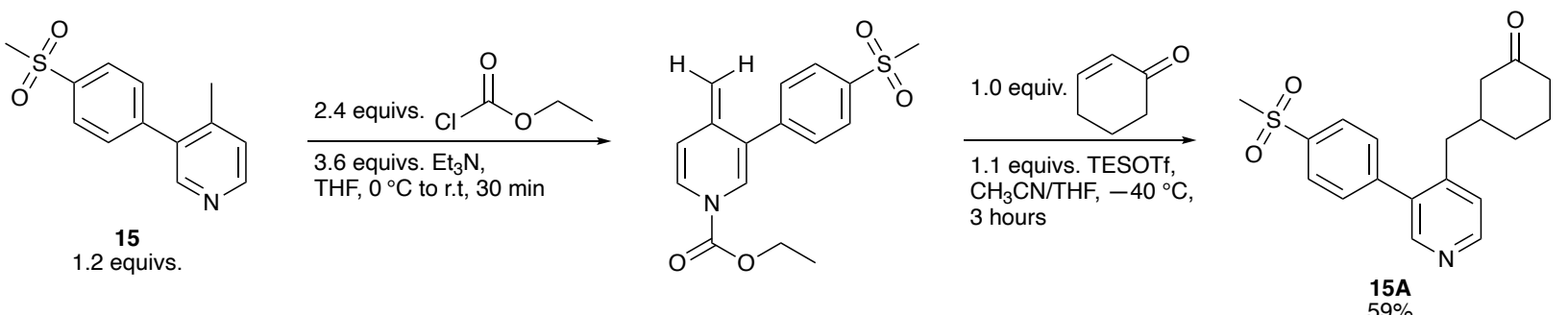

Pyridine 15 (95 mg, 1.2 equivs., $0.38 \mathrm{mmol}$ ) was converted to the corresponding ADHP following general procedure $\mathbf{A}$. Final product 15A was synthesized following general procedure B but using anhydrous THF (0.2 M) to dissolve the ADHP instead of $\mathrm{CH}_{3} \mathrm{CN}$ due to its poor solubility in $\mathrm{CH}_{3} \mathrm{CN}$, and 2-cyclohexen-1-one $(0.03 \mathrm{~mL}, 1.0$ equiv., $0.32 \mathrm{mmol})$ was used as the electrophile. The crude product was purified by flash column chromatography using EtOAc/hexanes to afford compound $15 \mathrm{~A}$ as colorless oil (64 $\mathrm{mg}, 59 \%$ yield).

Chromatography: $90 \%$ EtOAc in hexanes. $\left(R_{f}=0.17\right)$

1․ NMR $\quad\left(400 \mathrm{MHz}, \mathrm{CDCl}_{3}\right)$

$\delta 8.57(\mathrm{~d}, J=5.1 \mathrm{~Hz}, 1 \mathrm{H}), 8.42(\mathrm{~s}, 1 \mathrm{H}), 8.05(\mathrm{~d}, J=8.4 \mathrm{~Hz}, 2 \mathrm{H}), 7.50$ (d, $J=8.4$ $\mathrm{Hz}, 2 \mathrm{H}), 7.20(\mathrm{~d}, J=5.1 \mathrm{~Hz}, 2 \mathrm{H}), 3.14(\mathrm{~s}, 3 \mathrm{H}), 2.68(\mathrm{dd}, J=13.7,6.8 \mathrm{~Hz}, 1 \mathrm{H}), 2.59$ $(\mathrm{dd}, J=13.8,6.8 \mathrm{~Hz}, 1 \mathrm{H}), 2.35-2.26(\mathrm{~m}, 1 \mathrm{H}), 2.22-2.13(\mathrm{~m}, 2 \mathrm{H}), 1.99-1.88$ $(\mathrm{m}, 2 \mathrm{H}), 1.88-1.81(\mathrm{~m}, 1 \mathrm{H}), 1.74-1.65(\mathrm{~m}, 1 \mathrm{H}), 1.58-1.46(\mathrm{~m}, 1 \mathrm{H}), 1.23-1.12$ $(\mathrm{m}, 1 \mathrm{H})$

${ }^{13} \mathrm{C} \mathrm{NMR} \quad\left(100 \mathrm{MHz}, \mathrm{CDCl}_{3}\right)$

$\delta$ 210.4, 150.2, 149.7, 145.9, 143.8, 140.3, 136.3, 130.6, 127.9, 124.6, 47.6, 44.7, $41.2,40.0,39.0,31.1,25.0$.

IR Alpha-Platinum ATR, Bruker, diamond crystal $v=3013,2926,2864,1704,1653,1301,1090 \mathrm{~cm}^{-1}$.

HRMS ESI-TOF

$(\mathrm{M}+\mathrm{H})^{+}$Calcd for $\mathrm{C}_{19} \mathrm{H}_{22} \mathrm{NO}_{3} \mathrm{~S} 344.1315$; Found 344.1324. 
<smiles>Cc1ccncc1-c1cccc(C#N)c1</smiles>
1.2 equivs

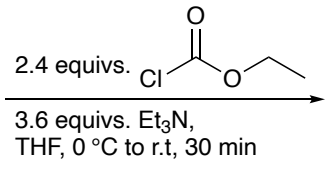

(1)
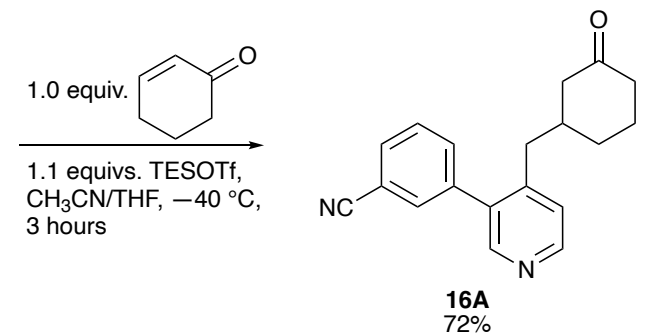

Pyridine 16 (233 mg, 1.2 equivs., $1.2 \mathrm{mmol}$ ) was converted to the corresponding ADHP following general procedure $\mathrm{A}$. Final product $16 \mathrm{~A}$ was synthesized following general procedure B but using anhydrous THF $(0.2 \mathrm{M})$ to dissolve the ADHP instead of $\mathrm{CH}_{3} \mathrm{CN}$ due to its poor solubility in $\mathrm{CH}_{3} \mathrm{CN}$, and 2-cyclohexen-1-one $(0.1 \mathrm{~mL}, 1.0$ equiv., $1.0 \mathrm{mmol})$ was used as the electrophile. The crude product was purified by flash column chromatography using EtOAc/hexanes/Et ${ }_{3} \mathrm{~N}$ to afford compound $16 \mathrm{~A}$ as white solid (209 mg, 72\% yield).

Melting point: $57-58^{\circ} \mathrm{C}$

Chromatography: $70 \%$ EtOAc in hexanes with $2 \% \mathrm{Et}_{3} \mathrm{~N} .\left(\mathrm{R}_{\mathrm{f}}=0.28\right)$

$\underline{1 \text { H NMR }} \quad\left(400 \mathrm{MHz}, \mathrm{CDCl}_{3}\right)$

$\delta 8.51(\mathrm{~d}, J=5.1 \mathrm{~Hz}, 1 \mathrm{H}), 8.36(\mathrm{~s}, 1 \mathrm{H}), 7.71-7.67(\mathrm{~m}, 1 \mathrm{H}), 7.59-7.54(\mathrm{~m}, 2 \mathrm{H})$, $7.52-7.48(\mathrm{~m}, 1 \mathrm{H}), 7.16(\mathrm{~d}, J=5.1 \mathrm{~Hz}, 1 \mathrm{H}), 2.62(\mathrm{dd}, 13.7,6.7 \mathrm{~Hz}, 1 \mathrm{H}), 2.56(\mathrm{dd}$, $J=13.7,7.0 \mathrm{~Hz}, 1 \mathrm{H}), 2.29-2.22(\mathrm{~m}, 1 \mathrm{H}), 2.18-2.09(\mathrm{~m}, 2 \mathrm{H}), 1.95-1.78(\mathrm{~m}$, $3 \mathrm{H}), 1.68-1.63(\mathrm{~m}, 1 \mathrm{H}), 1.54-1.42(\mathrm{~m}, 1 \mathrm{H}), 1.21-1.09(\mathrm{~m}, 1 \mathrm{H})$.

${ }^{13} \mathrm{C}$ NMR $\quad\left(100 \mathrm{MHz}, \mathrm{CDCl}_{3}\right)$

$\delta 210.2,150.2,149.5,145.9,139.1,135.8,133.9,132.8,131.6,129.6,124.5$, $118.3,113.0,47.5,41.1,39.7,38.8,30.8,24.8$.

IR Alpha-Platinum ATR, Bruker, diamond crystal $v=3029,2934,2866,2229,1706,1588,799 \mathrm{~cm}^{-1}$.

HRMS ESI-TOF

$(\mathrm{M}+\mathrm{H})^{+}$Calcd for $\mathrm{C}_{19} \mathrm{H}_{19} \mathrm{~N}_{2} \mathrm{O}$ 291.1492; Found 291.1497. 
<smiles>Cc1ccncc1C#CS</smiles>
1.2 equivs.

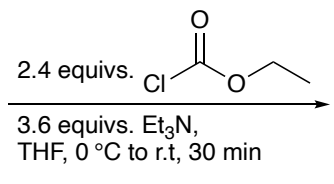

THF, $0^{\circ} \mathrm{C}$ to r.t, $30 \mathrm{~min}$
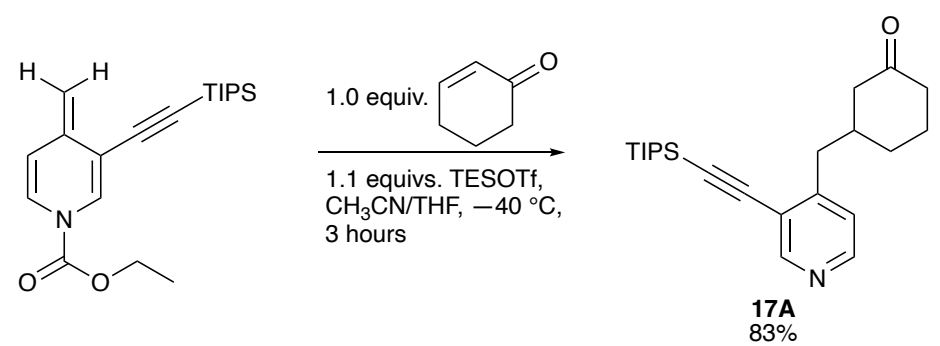

Pyridine 17 (164.1 mg, 1.2 equivs., $1.2 \mathrm{mmol}$ ) was converted to the corresponding ADHP following general procedure $\mathrm{A}$. Final product 17A was synthesized following general procedure B but using anhydrous THF $(0.2 \mathrm{M})$ to dissolve the ADHP instead of $\mathrm{CH}_{3} \mathrm{CN}$ due to its poor solubility in $\mathrm{CH}_{3} \mathrm{CN}$, and 2-cyclohexen-1-one $(0.1 \mathrm{~mL}, 1.0$ equiv. $1.0 \mathrm{mmol})$ was used as the electrophile. The crude product was purified by flash column chromatography using EtOAc/hexanes to afford 17A as light-yellow oil ( $154 \mathrm{mg}, 83 \%$ yield).

Chromatography: $30 \%$ EtOAc in hexanes. $\left(R_{f}=0.28\right)$

1H NMR $\quad\left(400 \mathrm{MHz}, \mathrm{CDCl}_{3}\right)$

$\delta 8.63(\mathrm{~s}, 1 \mathrm{H}), 8.39(\mathrm{~d}, J=5.1 \mathrm{~Hz}, 1 \mathrm{H}), 7.03(\mathrm{~d}, J=5.1 \mathrm{~Hz}, 1 \mathrm{H}), 2.86(\mathrm{dd}, J=12.9$, $7.0 \mathrm{~Hz}, 1 \mathrm{H}), 2.73(\mathrm{dd}, J=12.9,7.3 \mathrm{~Hz}, 1 \mathrm{H}), 2.37-2.30(\mathrm{~m}, 2 \mathrm{H}), 2.30-2.19(\mathrm{~m}$, $2 \mathrm{H}), 2.13-1.98(\mathrm{~m}, 2 \mathrm{H}), 1.89-1.81(\mathrm{~m}, 1 \mathrm{H}), 1.65-1.52(\mathrm{~m}, 1 \mathrm{H}), 1.47-1.36(\mathrm{~m}$, $1 \mathrm{H}), 1.17-1.08(\mathrm{~m}, 21 \mathrm{H})$.

${ }^{13} \mathrm{C} \mathrm{NMR} \quad\left(100 \mathrm{MHz}, \mathrm{CDCl}_{3}\right)$

$\delta 210.5,153.7,150.1,148.5,124.2,120.8,102.2,98.6,47.7,41.4,41.0,39.3,31.2$, 25.0, 18.8, 11.3.

$\underline{\text { IR }}$

Alpha-Platinum ATR, Bruker, diamond crystal

$v=3037,2941,2864,2155,1712,1584,1462,1225 \mathrm{~cm}^{-1}$.

HRMS ESI-TOF

$(\mathrm{M}+\mathrm{H})^{+}$Calcd for $\mathrm{C}_{23} \mathrm{H}_{36} \mathrm{NOSi} 370.2561$; Found 370.2652. 
<smiles>Cc1ccncc1/C=C/c1ccccc1</smiles>
18
18 equivs.
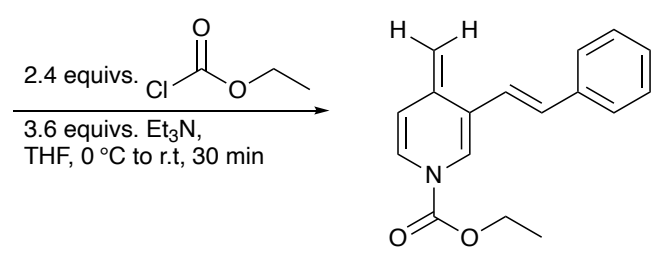
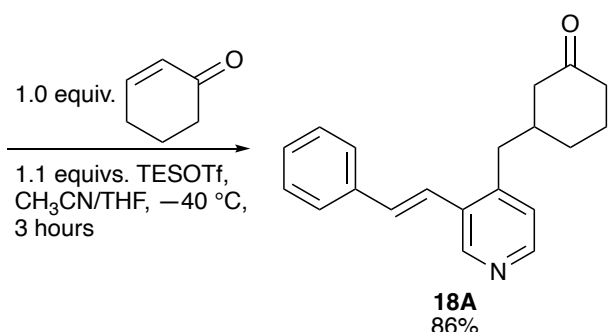

Pyridine 18 (47 mg, 1.2 equivs., $0.24 \mathrm{mmol}$ ) was converted to the corresponding ADHP following general procedure $\mathrm{A}$. Final product 18A was synthesized following general procedure B but using anhydrous THF $(0.2 \mathrm{M})$ to dissolve the ADHP instead of $\mathrm{CH}_{3} \mathrm{CN}$ due to its poor solubility in $\mathrm{CH}_{3} \mathrm{CN}$, and 2-cyclohexen-1-one $(0.02 \mathrm{~mL}, 1.0$ equiv. $0.2 \mathrm{mmol})$ was used as the electrophile. The crude product was purified by flash column chromatography using EtOAc/hexanes/Et ${ }_{3} \mathrm{~N}$ to afford compound $18 \mathrm{~A}$ as light-yellow oil (50 mg, $86 \%$ yield).

Chromatography: $50 \%$ EtOAc in hexanes with $2 \% \mathrm{Et}_{3} \mathrm{~N} .\left(\mathrm{R}_{\mathrm{f}}=0.29\right)$

1 NMR $\quad\left(400 \mathrm{MHz}, \mathrm{CDCl}_{3}\right)$

$\delta 8.78(\mathrm{~s}, 1 \mathrm{H}), 8.41(\mathrm{~d}, J=4.9 \mathrm{~Hz}, 1 \mathrm{H}), 7.53-7.50(\mathrm{~m}, 2 \mathrm{H}), 7.42-7.37(\mathrm{~m}, 2 \mathrm{H})$, $7.34-7.29(\mathrm{~m}, 1 \mathrm{H}), 7.22-7.17(\mathrm{~m}, 1 \mathrm{H}), 7.07-7.02(\mathrm{~m}, 2 \mathrm{H}), 2.81-2.70(\mathrm{~m}, 2 \mathrm{H})$, $2.44-2.34(\mathrm{~m}, 2 \mathrm{H}), 2.31-2.22(\mathrm{~m}, 1 \mathrm{H}), 2.13-2.08(\mathrm{~m}, 2 \mathrm{H}), 2.08-2.02(\mathrm{~m}, 1 \mathrm{H})$, $1.88(\mathrm{~d}, \mathrm{~J}=14.2 \mathrm{~Hz}, 1 \mathrm{H}), 1.67-1.55(\mathrm{~m}, 1 \mathrm{H}), 1.47-1.38(\mathrm{~m}, 1 \mathrm{H})$.

${ }^{13}$ C NMR $\quad\left(100 \mathrm{MHz}, \mathrm{CDCl}_{3}\right)$

$\delta 210.7,148.4,147.9,145.6,136.9,132.8,132.7,129.0,128.4,126.9,124.9$, $122.8,47.9,41.4,39.7,39.6,31.2,25.0$.

IR Alpha-Platinum ATR, Bruker, diamond crystal $v=3024,2932,2870,1706,1586,1448,963 \mathrm{~cm}^{-1}$.

HRMS ESI-TOF

$(\mathrm{M}+\mathrm{H})^{+}$Calcd for $\mathrm{C}_{20} \mathrm{H}_{22} \mathrm{NO}$ 292.1696; Found 292.1699. 

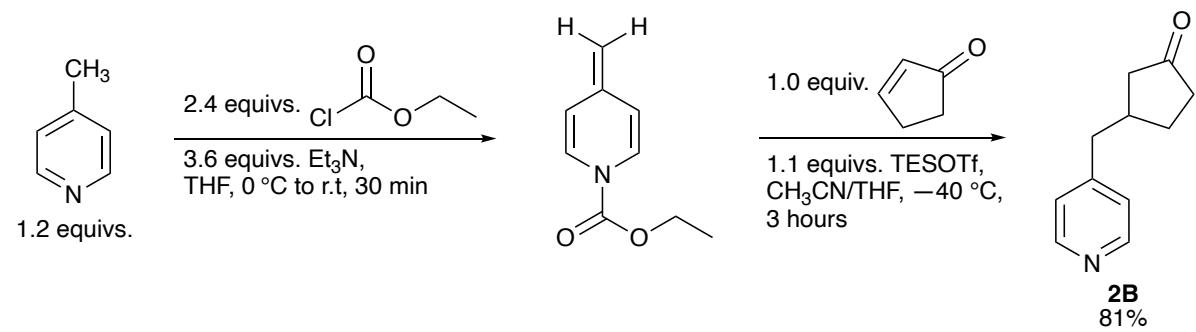

4-Picoline ( $0.12 \mathrm{~mL}, 1.2$ equivs., $1.2 \mathrm{mmol})$ was converted to the corresponding ADHP following general procedure $\mathbf{A}$. Final product $2 \mathrm{~B}$ was synthesized following general procedure B but using anhydrous THF $(0.2 \mathrm{M})$ to dissolve the ADHP instead of $\mathrm{CH}_{3} \mathrm{CN}$ due to its poor solubility in $\mathrm{CH}_{3} \mathrm{CN}$, and 2-cyclopenten-1-one $(0.1 \mathrm{~mL}, 1.0$ equiv. $1.0 \mathrm{mmol})$ was used as the electrophile. The crude product was purified by flash column chromatography using EtOAc/hexanes/Et ${ }_{3} \mathrm{~N}$ to afford compound 2B as light-yellow oil (141 mg, 81\% yield).

Chromatography: $50 \%$ EtOAc in hexanes with $2 \% \mathrm{Et}_{3} \mathrm{~N} .\left(\mathrm{R}_{\mathrm{f}}=0.24\right)$

1․ NMR $\quad\left(400 \mathrm{MHz}, \mathrm{CDCl}_{3}\right)$

$\delta 8.52(\mathrm{~d}, J=5.9 \mathrm{~Hz}, 2 \mathrm{H}), 7.10(\mathrm{~d}, J=5.9 \mathrm{~Hz}, 2 \mathrm{H}), 2.76(\mathrm{dd}, J=13.6,7.6 \mathrm{~Hz}, 1 \mathrm{H})$, $2.72(\mathrm{dd}, J=13.6,6.7 \mathrm{~Hz}, 1 \mathrm{H}), 2.55-2.42(\mathrm{~m}, 1 \mathrm{H}), 2.39-2.28(\mathrm{~m}, 2 \mathrm{H}), 2.22-$ $2.08(\mathrm{~m}, 2 \mathrm{H}), 1.94-1.86(\mathrm{~m}, 1 \mathrm{H}), 1.67-1.55(\mathrm{~m}, 1 \mathrm{H})$.

${ }^{13} \mathrm{C}$ NMR $\quad\left(100 \mathrm{MHz}, \mathrm{CDCl}_{3}\right)$

$\delta$ 218.2, 150.0, 149.0, 124.3, 44.9, 41.0, 38.3, 38.0, 29.2.

IR Alpha-Platinum ATR, Bruker, diamond crystal $v=3024,2960,2928,1735,1601,1415 \mathrm{~cm}^{-1}$.

HRMS ESI-TOF

$(\mathrm{M}+\mathrm{H})^{+}$Calcd for $\mathrm{C}_{11} \mathrm{H}_{14} \mathrm{NO}$ 176.1070; Found 176.1077. 

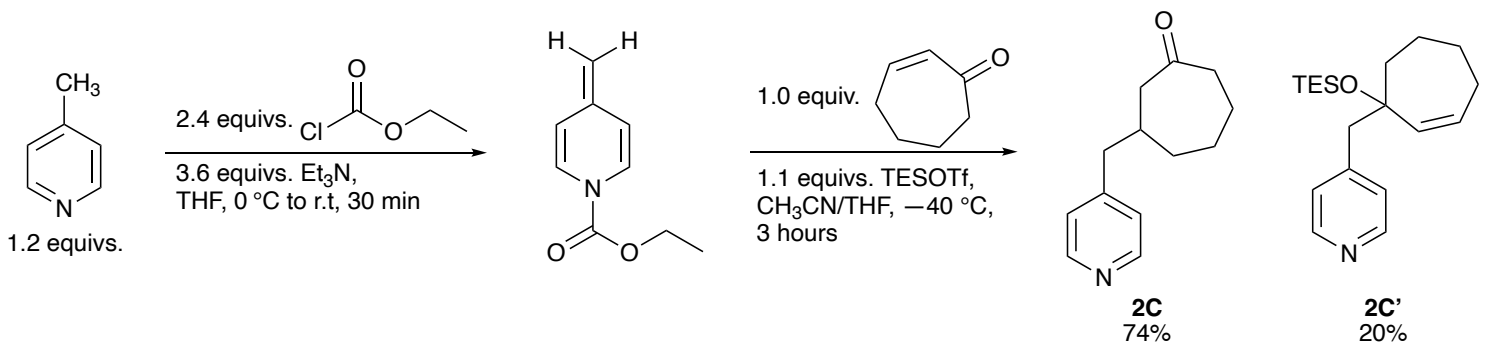

4-Picoline ( $0.23 \mathrm{~mL}, 1.2$ equivs., $2.4 \mathrm{mmol})$ was converted to the corresponding ADHP following general procedure $\mathbf{A}$. Final products $2 \mathrm{C}$ and $2 \mathrm{C}^{\prime}$ were synthesized following general procedure $B$ but using anhydrous THF $(0.2 \mathrm{M})$ to dissolve the ADHP instead of $\mathrm{CH}_{3} \mathrm{CN}$ due to its poor solubility in $\mathrm{CH}_{3} \mathrm{CN}$, and 2-cyclohepten-1-one $(0.22 \mathrm{~mL}, 1.0$ equiv. $2.0 \mathrm{mmol})$ was used as the electrophile. The crude mixture was purified by flash column chromatography using EtOAc/hexanes/Et ${ }_{3} \mathrm{~N}$ to afford compound $2 \mathrm{C}$ as light-yellow oil (304 mg, 74\% yield) alongside undesired 1,2-addition product 2C' as colorless oil (127 mg, 20\% yield).

Chromatography: $50 \%$ EtOAc in hexanes with $2 \% \mathrm{Et}_{3} \mathrm{~N} .\left(\mathrm{R}_{\mathrm{f}}=0.27\right)$

Data for 1,4-addition product (2C):

${ }^{1} \mathrm{H}$ NMR $\quad\left(400 \mathrm{MHz}, \mathrm{CDCl}_{3}\right)$

$\delta 8.45(\mathrm{~d}, J=6.0 \mathrm{~Hz}, 2 \mathrm{H}), 7.07(\mathrm{~d}, J=6.0 \mathrm{~Hz}, 2 \mathrm{H}), 2.59-2.48(\mathrm{~m}, 2 \mathrm{H}), 2.46-$ $2.34(\mathrm{~m}, 4 \mathrm{H}), 2.01-1.94(\mathrm{~m}, 1 \mathrm{H}), 1.89-1.77(\mathrm{~m}, 3 \mathrm{H}), 1.61-1.51(\mathrm{~m}, 1 \mathrm{H}), 1.38$ $-1.18(\mathrm{~m}, 2 \mathrm{H})$.

${ }^{13} \mathrm{C} \mathrm{NMR} \quad\left(100 \mathrm{MHz}, \mathrm{CDCl}_{3}\right)$

$\delta 213.5,149.9,148.9,124.6,49.5,44.0,42.9,37.1,36.4,28.4,24.3$.

IR Alpha-Platinum ATR, Bruker, diamond crystal $v=3021,2925,2856,1695,1600,1414 \mathrm{~cm}^{-1}$.

HRMS ESI-TOF

$(\mathrm{M}+\mathrm{H})^{+}$Calcd for $\mathrm{C}_{13} \mathrm{H}_{18} \mathrm{NO} 204.1383$; Found 204.1394.

Data for 1,2-addition product (2C'):

${ }^{1} \mathrm{H}$ NMR $\quad\left(400 \mathrm{MHz}, \mathrm{CDCl}_{3}\right)$

$\delta 8.44(\mathrm{~d}, J=5.2 \mathrm{~Hz}, 2 \mathrm{H}), 7.16(\mathrm{~d}, J=5.2 \mathrm{~Hz}, 2 \mathrm{H}), 5.67-5.60(\mathrm{~m}, 1 \mathrm{H}), 5.45-$ $5.41(\mathrm{~m}, 1 \mathrm{H}), 2.88(\mathrm{~d}, J=12.8 \mathrm{~Hz}, 1 \mathrm{H}), 2.73(\mathrm{~d}, J=12.8 \mathrm{~Hz}, 1 \mathrm{H}), 2.25-2.18(\mathrm{~m}$, 1H), $2.16-2.05(\mathrm{~m}, 1 \mathrm{H}), 1,83-1.68(\mathrm{~m}, 4 \mathrm{H}), 1.67-1.48(\mathrm{~m}, 2 \mathrm{H}), 0.85(\mathrm{t}, J=7.9$ $\mathrm{Hz}, 9 \mathrm{H}), 0.52$ (q, $J=7.9 \mathrm{~Hz}, 6 \mathrm{H})$.

${ }^{13} \mathrm{C} \mathrm{NMR} \quad\left(100 \mathrm{MHz}, \mathrm{CDCl}_{3}\right)$

$\delta 148.9,147.3,139.9,128.7,126.6,79.2,46.5,39.9,28.1,27.6,24.5,7.2,6.8$. 

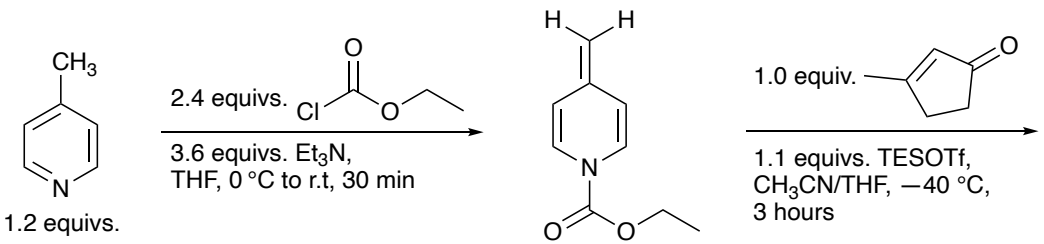

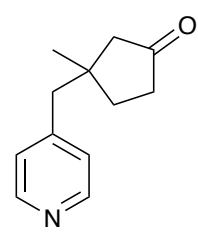

$\stackrel{2 \mathrm{D}}{48 \%}$

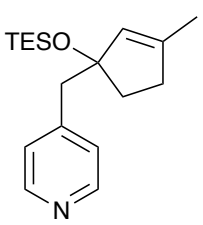

$2 D^{\prime}$
$6 \%$

4-Picoline $(0.12 \mathrm{~mL}, 1.2$ equivs., $1.2 \mathrm{mmol})$ was converted to the corresponding ADHP following general procedure A. Final products 2D and 2D' were synthesized following general procedure B but using anhydrous THF $(0.2 \mathrm{M})$ to dissolve the ADHP instead of $\mathrm{CH}_{3} \mathrm{CN}$ due to its poor solubility in $\mathrm{CH}_{3} \mathrm{CN}$, and 3-methyl-2-cyclopenten-1-one $(0.1 \mathrm{~mL}, 1.0$ equiv. $1.0 \mathrm{mmol})$ was used as the electrophile. The crude mixture was purified by flash column chromatography using EtOAc/hexanes/Et ${ }_{3} \mathrm{~N}$ to afford compound 2D as light-yellow oil (92 mg, 48\% yield) alongside undesired 1,2-addition product 2D' as colorless oil (19 $\mathrm{mg}, 6 \%$ yield).

Chromatography: $50 \%$ EtOAc in hexanes with $2 \% \mathrm{Et}_{3} \mathrm{~N} .\left(\mathrm{R}_{\mathrm{f}}=0.24\right)$

Data for 1,4-addition product (2D):

1H NMR $\quad\left(400 \mathrm{MHz}, \mathrm{CDCl}_{3}\right)$

$\delta 8.51(\mathrm{~d}, J=5.9 \mathrm{~Hz}, 2 \mathrm{H}), 7.05(\mathrm{~d}, J=5.9 \mathrm{~Hz}, 2 \mathrm{H}), 2.73-2.64(\mathrm{~m}, 2 \mathrm{H}), 2.32-$

$2.27(\mathrm{~m}, 2 \mathrm{H}), 2.21-2.15(\mathrm{~m}, 1 \mathrm{H}), 2.02-1.97(\mathrm{~m}, 1 \mathrm{H}), 1.95-1.86(\mathrm{~m}, 1 \mathrm{H}), 1.79$

$-1.73(\mathrm{~m}, 1 \mathrm{H}), 1.02(\mathrm{~s}, 3 \mathrm{H})$.

${ }^{13} \mathrm{C} \mathrm{NMR} \quad\left(100 \mathrm{MHz}, \mathrm{CDCl}_{3}\right)$

$\delta 218.1,149.6,146.9,125.4,51.6,46.7,40.3,36.4,34.7,25.2$.

IR Alpha-Platinum ATR, Bruker, diamond crystal $v=3026,2957,2928,1735,1599,1415 \mathrm{~cm}^{-1}$.

HRMS ESI-TOF

$(\mathrm{M}+\mathrm{H})^{+}$Calcd for $\mathrm{C}_{12} \mathrm{H}_{16} \mathrm{NO}$ 190.1227; Found 190.1238.

Data for 1,2-addition product (2D'):

1H NMR $\quad\left(400 \mathrm{MHz}, \mathrm{CDCl}_{3}\right)$

$\delta 8.45(\mathrm{~d}, J=5.9 \mathrm{~Hz}, 2 \mathrm{H}), 7.15(\mathrm{~d}, J=5.9 \mathrm{~Hz}, 2 \mathrm{H}), 5.25-5.24(\mathrm{~m}, 1 \mathrm{H}), 2.80(\mathrm{q}, J$ $=12.8 \mathrm{~Hz}, 2 \mathrm{H}), 2.35-2.27(\mathrm{~m}, 1 \mathrm{H}), 2.11-2.01(\mathrm{~m}, 2 \mathrm{H}), 1.91-1.82(\mathrm{~m}, 1 \mathrm{H}), 1.71$ $(\mathrm{s}, 3 \mathrm{H}), 0.85(\mathrm{t}, J=7.9 \mathrm{~Hz}, 9 \mathrm{H}), 0.46(\mathrm{q}, J=7.9 \mathrm{~Hz}, 6 \mathrm{H})$.

${ }^{13} \mathrm{C}$ NMR $\quad\left(100 \mathrm{MHz}, \mathrm{CDCl}_{3}\right)$

$\delta 149.1,147.9,143.6,130.5,126.2,87.8,48.7,39.2,35.3,16.9,7.1,6.4$. 
<smiles>Cc1ccncc1</smiles>

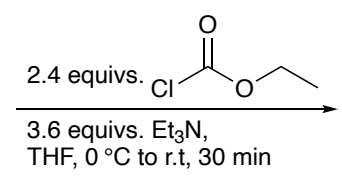

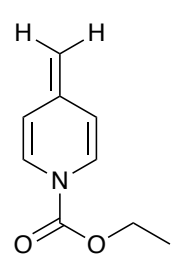

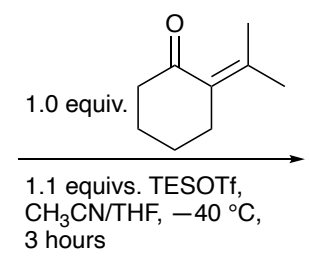

4-Picoline ( $0.12 \mathrm{~mL}, 1.2$ equivs., $1.2 \mathrm{mmol})$ was converted to the corresponding ADHP following general procedure A. Fnal product 2E was synthesized following general procedure B but using anhydrous THF $(0.2 \mathrm{M})$ to dissolve the ADHP instead of $\mathrm{CH}_{3} \mathrm{CN}$ due to its poor solubility in $\mathrm{CH}_{3} \mathrm{CN}$, and 2-isopropylidene-cyclohexanone $(0.15 \mathrm{~mL}, 1.0$ equiv. $1.0 \mathrm{mmol})$ was used as the electrophile. The crude product was purified by flash column chromatography using EtOAc/hexanes to afford compound $2 \mathrm{E}$ as colorless oil (129 mg, $58 \%$ yield).

Chromatography: $70 \%$ EtOAc in hexanes $\left(R_{f}=0.37\right)$

1 NMR $\quad\left(400 \mathrm{MHz}, \mathrm{CDCl}_{3}\right)$

$\delta 8.46(\mathrm{~d}, J=6.0 \mathrm{~Hz}, 2 \mathrm{H}), 7.02(\mathrm{~d}, J=6.0 \mathrm{~Hz}, 2 \mathrm{H}), 3.02(\mathrm{~d}, J=12.6 \mathrm{~Hz}, 1 \mathrm{H}), 2.58$ $(\mathrm{d}, J=12.6 \mathrm{~Hz}, 1 \mathrm{H}), 2.36-2.30(\mathrm{~m}, 1 \mathrm{H}), 2.29-2.20(\mathrm{~m}, 1 \mathrm{H}), 2.19-2.13(\mathrm{~m}, 1 \mathrm{H})$, $2.09-2.03(\mathrm{~m}, 2 \mathrm{H}), 1.93-1.89(\mathrm{~m}, 1 \mathrm{H}), 1.75-1.61(\mathrm{~m}, 1 \mathrm{H}), 1.61-1.45(\mathrm{~m}, 2 \mathrm{H})$, $1.03(\mathrm{~s}, 3 \mathrm{H}), 0.92(\mathrm{~s}, 3 \mathrm{H})$.

${ }^{13} \mathrm{C} \mathrm{NMR} \quad\left(100 \mathrm{MHz}, \mathrm{CDCl}_{3}\right)$

$\delta$ 213.1, 149.3, 148.5, 126.1, 58.0, 45.0, 44.5, 35.9, 29.7, 28.8, 26.1, 26.0, 24.2.

IR Alpha-Platinum ATR, Bruker, diamond crystal $v=3024,2935,2866,1703,1598,1448 \mathrm{~cm}^{-1}$.

HRMS ESI-TOF

$(\mathrm{M}+\mathrm{H})^{+}$Calcd for $\mathrm{C}_{15} \mathrm{H}_{22} \mathrm{NO} 232.1696$; Found 232.1703. 

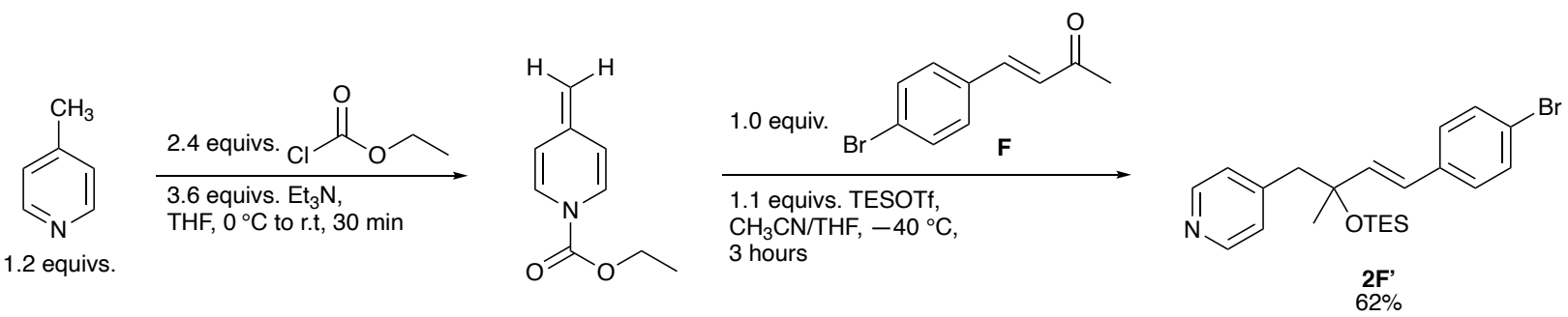

4-Picoline ( $0.12 \mathrm{~mL}, 1.2$ equivs., $1.2 \mathrm{mmol}$ ) was converted to the corresponding ADHP following general procedure $A$. Final product $2 \mathrm{~F}$ was synthesized following general procedure B but using anhydrous THF $(0.2 \mathrm{M})$ to dissolve the ADHP instead of $\mathrm{CH}_{3} \mathrm{CN}$ due to its poor solubility in $\mathrm{CH}_{3} \mathrm{CN}$, and compound $\mathbf{F}(225.1 \mathrm{mg}, 1.0$ equiv. $1.0 \mathrm{mmol})$ was used as the electrophile. The crude product was purified by flash column chromatography using EtOAc/hexanes to afford 1,2-addition product 2F' as colorless oil (270 mg, 62\% yield).

Chromatography: $30 \%$ EtOAc in hexanes. $\left(R_{f}=0.36\right)$

1'H NMR $\quad\left(300 \mathrm{MHz}, \mathrm{CDCl}_{3}\right)$

$\delta 8.47(\mathrm{~d}, J=6.0 \mathrm{~Hz}, 2 \mathrm{H}), 7.43(\mathrm{~d}, J=8.4 \mathrm{~Hz}, 2 \mathrm{H}), 7.17(\mathrm{~d}, J=8.4 \mathrm{~Hz}, 2 \mathrm{H}), 7.12$

$(\mathrm{d}, J=6.0 \mathrm{~Hz}, 2 \mathrm{H}), 6.34(\mathrm{~d}, J=16.1 \mathrm{~Hz}, 1 \mathrm{H}), 6.17(\mathrm{~d}, J=16.1 \mathrm{~Hz}, 1 \mathrm{H}), 2.82(\mathrm{~s}$,

2H), $1.43(\mathrm{~s}, 3 \mathrm{H}), 0.89(\mathrm{t}, J=8.0 \mathrm{~Hz}, 9 \mathrm{H}), 0.65(\mathrm{q}, J=8.0 \mathrm{~Hz}, 6 \mathrm{H})$.

${ }^{13} \mathrm{C} \mathrm{NMR} \quad\left(75 \mathrm{MHz}, \mathrm{CDCl}_{3}\right)$

$\delta 149.2,146.7,137.1,135.9,131.9,128.0,126.9,126.4,121.5,74.9,50.5,27.1$, $7.2,6.8$.

IR Alpha-Platinum ATR, Bruker, diamond crystal $v=3027,2953,2874,1599,1005,788 \mathrm{~cm}^{-1}$.

HRMS ESI-TOF

$(\mathrm{M}+\mathrm{H})^{+}$Calcd for $\mathrm{C}_{22} \mathrm{H}_{31}$ BrNOSi 432.1353; Found 432.1379. 

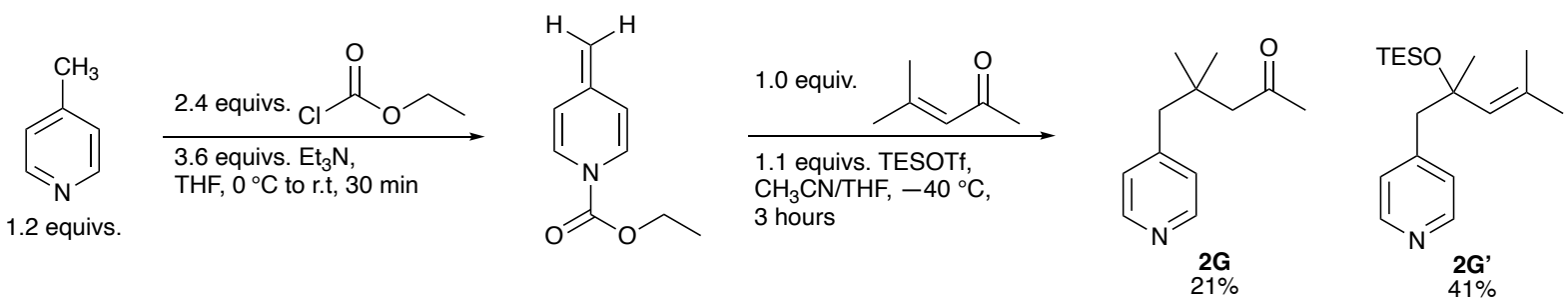

4-Picoline ( $0.12 \mathrm{~mL}, 1.2$ equivs., $1.2 \mathrm{mmol})$ was converted to the corresponding ADHP following general procedure A. Final products $\mathbf{2 G}$ and 2G' were synthesized following general procedure $\mathbf{B}$ but using anhydrous THF $(0.2 \mathrm{M})$ to dissolve the ADHP instead of $\mathrm{CH}_{3} \mathrm{CN}$ due to its poor in $\mathrm{CH}_{3} \mathrm{CN}$, and mesityl oxide $(0.12 \mathrm{~mL}, 1.0$ equiv. $1.0 \mathrm{mmol})$ was used as the electrophile. The crude mixture was purified by flash column chromatography using EtOAc/hexanes/Et ${ }_{3} \mathrm{~N}$ to afford compound 1,4-addition product $\mathbf{2} \mathbf{G}$ as colorless oil (41 $\mathrm{mg}, 21 \%$ yield) alongside undesired 1,2-addition product 2G' as colorless oil (126 mg, $41 \%$ yield).

Chromatography: $50 \%$ EtOAc in hexanes with $2 \% \mathrm{Et}_{3} \mathrm{~N} .\left(\mathrm{R}_{\mathrm{f}}=0.33\right)$

Data for 1,4-addition product (2G):

1H NMR $\quad\left(400 \mathrm{MHz}, \mathrm{CDCl}_{3}\right)$

$\delta 8.47(\mathrm{~d}, J=5.6 \mathrm{~Hz}, 2 \mathrm{H}), 7.05(\mathrm{~d}, J=5.6 \mathrm{~Hz}, 2 \mathrm{H}), 2.70(\mathrm{~s}, 2 \mathrm{H}), 2.27(\mathrm{~s}, 2 \mathrm{H}), 2.11$ $(\mathrm{s}, 3 \mathrm{H}), 1.00(\mathrm{~s}, 6 \mathrm{H})$.

${ }^{13} \mathrm{C} \mathrm{NMR} \quad\left(100 \mathrm{MHz}, \mathrm{CDCl}_{3}\right)$

$\delta 208.5,149.5,147.9,126.1,52.9,46.6,34.4,32.5,27.6$.

IR Alpha-Platinum ATR, Bruker, diamond crystal

$v=3069,3024,2960,2929,1709,1600,1416 \mathrm{~cm}^{-1}$.

HRMS ESI-TOF

$(\mathrm{M}+\mathrm{H})^{+}$Calcd for $\mathrm{C}_{12} \mathrm{H}_{18} \mathrm{NO}$ 192.1383; Found 192.1390.

Data for 1,2-addition product (2G'):

1H NMR (400 MHz, $\left.\mathrm{CDCl}_{3}\right)$

$\delta 8.41(\mathrm{~d}, J=6.0 \mathrm{~Hz}, 2 \mathrm{H}), 7.09(\mathrm{~d}, J=6.0 \mathrm{~Hz}, 2 \mathrm{H}), 5.16-5.14(\mathrm{~m}, 1 \mathrm{H}), 2.86(\mathrm{~d}, J$

$=12.8 \mathrm{~Hz}, 1 \mathrm{H}), 2.71(\mathrm{~d}, J=12.8 \mathrm{~Hz}, 1 \mathrm{H}), 1.68(\mathrm{~d}, J=1.2 \mathrm{~Hz}, 3 \mathrm{H}), 1.64(\mathrm{~d}, J=1.2$

$\mathrm{Hz}, 3 \mathrm{H}), 1.26(\mathrm{~s}, 3 \mathrm{H}), 0.85(\mathrm{t}, J=8.0 \mathrm{~Hz}, 9 \mathrm{H}), 0.50(\mathrm{q}, J=8.0 \mathrm{~Hz}, 6 \mathrm{H})$.

${ }^{13} \mathrm{C} \mathrm{NMR} \quad\left(100 \mathrm{MHz}, \mathrm{CDCl}_{3}\right)$

$\delta 148.9,147.5,133.9,131.6,126.4,75.0,49.7,29.6,27.3,19.0,7.1,6.7$. 

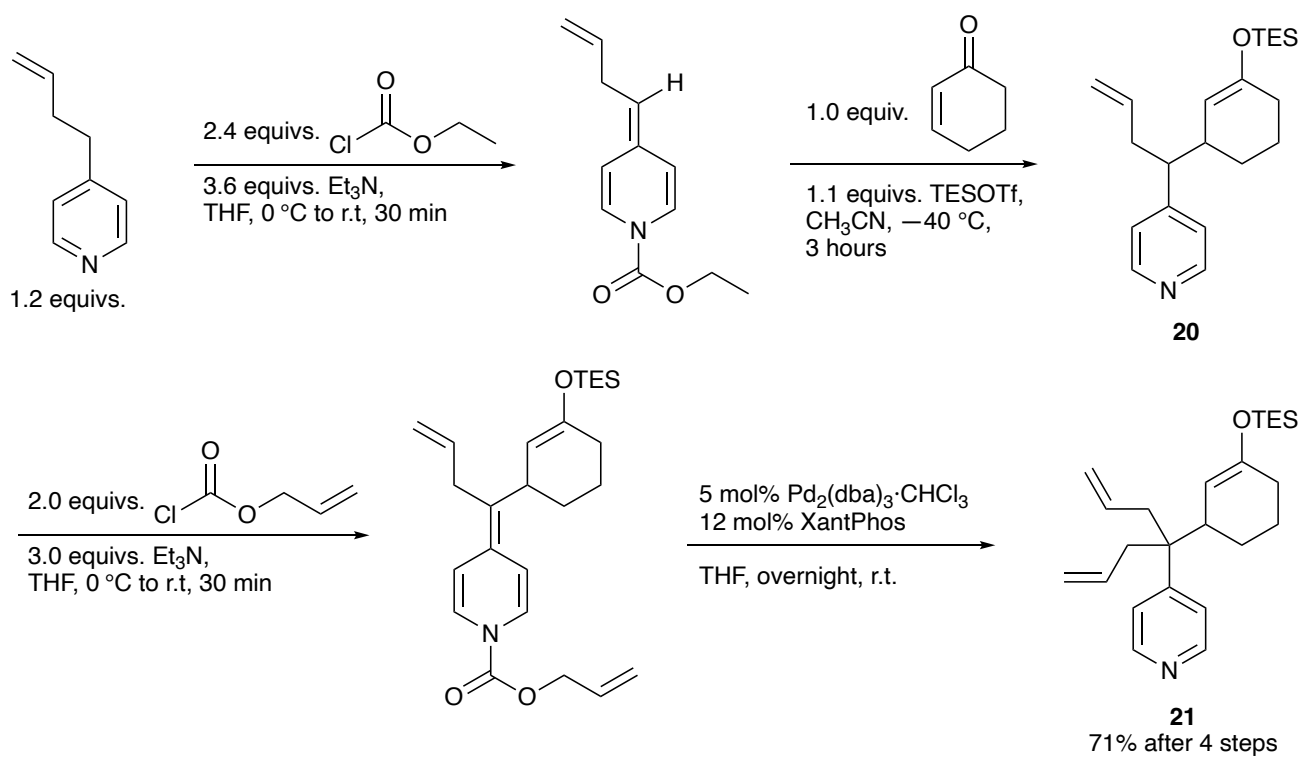

4-(3-Buten-1-yl)-pyridine (80 mg, 1.2 equivs., $0.6 \mathrm{mmol}$ ) was converted to the corresponding ADHP following general procedure $\mathbf{A}$.

A dry $25 \mathrm{~mL}$ round-bottomed flask was charged with a $0.2 \mathrm{M}$ solution of 2-cyclohexen-1one $(0.05 \mathrm{~mL}, 1.0$ equiv., $0.5 \mathrm{mmol})$ in anhydrous $\mathrm{CH}_{3} \mathrm{CN}$. The solution was cooled to $-40{ }^{\circ} \mathrm{C}$ and TESOTf $(0.13 \mathrm{~mL}, 1.1$ equivs., $0.55 \mathrm{mmol})$ was added dropwise. The resulting mixture was stirred for 15 minutes. The freshly prepared ADHP was dissolved in anhydrous $\mathrm{CH}_{3} \mathrm{CN}(0.2 \mathrm{M})$ and added dropwise to the above reaction mixture while maintaining the temperature at $-40^{\circ} \mathrm{C}$. The mixture was stirred under $-40{ }^{\circ} \mathrm{C}$ for 3 hours. The reaction mixture was placed in an ice-water bath and quenched with $\mathrm{pH} 7$ buffer $(10 \mathrm{~mL})$ followed by 5 drops of triethylamine. The resulting mixture was stirred vigorously for 30 minutes and extracted with EtOAc $(4 \times 10 \mathrm{~mL})$. The combined organic layer was washed with brine, dried with $\mathrm{MgSO}_{4}$, filtered through a cotton plug, and the filtrate was concentrated using a rotary evaporator. Crude product $\mathbf{2 0}$ was obtained and used for next step without further purification.

Compound 20 was converted into its corresponding ADHP following general procedure A using allyl chloroformate $(0.12 \mathrm{~mL}, 2.0$ equivs., $1.0 \mathrm{mmol})$ instead of ethyl chloroformate. To another dry $25 \mathrm{~mL}$ round-bottomed flask containing $3 \mathrm{~mL}$ of anhydrous THF were added Pd${ }_{2}(\mathrm{dba})_{3} \cdot \mathrm{CHCl}_{3}(25.9 \mathrm{mg}, 5 \mathrm{~mol} \%, 0.025 \mathrm{mmol}$ ) and XantPhos (34.8 mg, $12 \mathrm{~mol} \%, 0.06 \mathrm{mmol}$ ). The mixture was stirred for 30 minutes at ambient temperature. The second ADHP intermediate prepared from 20 was dissolved in anhydrous THF $(3 \mathrm{~mL})$ and added dropwise to the palladium catalyst solution. The resulting mixture was stirred at ambient temperature overnight, then concentrated using a rotary evaporator. The crude product was purified by flash column chromatography using EtOAc/hexanes to afford product 21 as colorless oil (137 mg, 71\% overall yield).

Data for compound 21:

Chromatography: $30 \%$ EtOAc in hexanes. $\left(R_{f}=0.28\right)$

1․ NMR $\quad\left(400 \mathrm{MHz}, \mathrm{CDCl}_{3}\right)$

$\delta 8.50(\mathrm{~d}, J=6.2 \mathrm{~Hz}, 2 \mathrm{H}), 7.23(\mathrm{~d}, J=6.2 \mathrm{~Hz}, 2 \mathrm{H}), 5.78-5.69(\mathrm{~m}, 1 \mathrm{H}), 5.67-$ $5.57(\mathrm{~m}, 1 \mathrm{H}), 5.13-5.01(\mathrm{~m}, 4 \mathrm{H}), 4.86(\mathrm{~s}, 1 \mathrm{H}), 2.65-2.52(\mathrm{~m}, 5 \mathrm{H}), 1.87-1.84$ $(\mathrm{m}, 2 \mathrm{H}), 1.68-1.60(\mathrm{~m}, 2 \mathrm{H}), 1.48-1.38(\mathrm{~m}, 1 \mathrm{H}), 1.07-0.97(\mathrm{~m}, 1 \mathrm{H}), 0.94(\mathrm{t}, J=$ $8.0 \mathrm{~Hz}, 9 \mathrm{H}), 0.60(\mathrm{q}, J=8.0 \mathrm{~Hz}, 6 \mathrm{H})$. 
${ }^{13} \mathrm{C} \mathrm{NMR} \quad\left(100 \mathrm{MHz}, \mathrm{CDCl}_{3}\right)$

$\delta 154.1,152.4,149.4,134.7,134.5,123.3,118.2,118.1,104.5,46.6,43.3,39.6$, $38.5,29.8,24.1,22.7,6.8,5.3$.

IR Alpha-Platinum ATR, Bruker, diamond crystal

$v=3076,3019,2935,1657,1594,1192 \mathrm{~cm}^{-1}$.

HRMS ESI-TOF

$(\mathrm{M}+\mathrm{H})^{+}$Calcd for $\mathrm{C}_{24} \mathrm{H}_{38} \mathrm{NOSi} 384.2717$; Found 384.2721. 

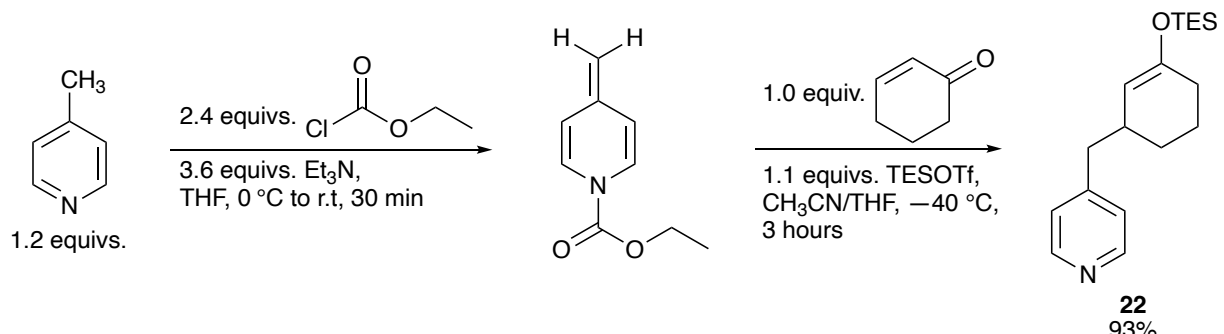

4-Picoline $(0.12 \mathrm{~mL}, 1.2$ equivs., $1.2 \mathrm{mmol}$ ) was converted to the corresponding ADHP following general procedure $A$.

A dry $25 \mathrm{~mL}$ round-bottomed flask was charged with a $0.2 \mathrm{M}$ solution of 2-cyclohexen-1one $(0.1 \mathrm{~mL}, 1.0$ equiv., $1.0 \mathrm{mmol})$ in anhydrous $\mathrm{CH}_{3} \mathrm{CN}$. The solution was cooled to $-40{ }^{\circ} \mathrm{C}$ and TESOTf $(0.25 \mathrm{~mL}, 1.1$ equivs., $1.1 \mathrm{mmol})$ was added dropwise. The resulting mixture was stirred for 15 minutes. The freshly prepared ADHP was dissolved in anhydrous THF $(0.2 \mathrm{M})$ and added dropwise to the above reaction mixture while maintaining the temperature at $-40^{\circ} \mathrm{C}$. The mixture was stirred under $-40{ }^{\circ} \mathrm{C}$ for 3 hours. The reaction mixture was placed in an ice-water bath and quenched with $\mathrm{pH} 7$ buffer $(10 \mathrm{~mL})$ followed by 5 drops of triethylamine. The resulting mixture was stirred vigorously for 30 minutes and extracted with EtOAc $(4 \times 10 \mathrm{~mL})$. The combined organic layer was washed with brine, dried with $\mathrm{MgSO}_{4}$, filtered through a cotton plug, and the filtrate was concentrated using a rotary evaporator. The crude reaction mixture was purified by flash column chromatography using EtOAc/hexanes to afford product 22 as colorless oil (281 mg, 93\% yield).

Chromatography: $20 \%$ EtOAc in hexanes. $\left(R_{f}=0.33\right)$

1․ NMR $\quad\left(400 \mathrm{MHz}, \mathrm{CDCl}_{3}\right)$

$\delta 8.46(\mathrm{~d}, J=6.0 \mathrm{~Hz}, 2 \mathrm{H}), 7.05(\mathrm{~d}, J=6.0 \mathrm{~Hz}, 2 \mathrm{H}), 4.68-4.66(\mathrm{~m}, 1 \mathrm{H}), 2.53-$ $2.50(\mathrm{~m}, 2 \mathrm{H}), 2.47-2.39(\mathrm{~m}, 1 \mathrm{H}), 2.26-1.91(\mathrm{~m}, 2 \mathrm{H}), 1.78-1.69(\mathrm{~m}, 1 \mathrm{H}), 1.64$ $-1.56(\mathrm{~m}, 1 \mathrm{H}), 1.56-1.46(\mathrm{~m}, 1 \mathrm{H}), 1.16-1.05(\mathrm{~m}, 1 \mathrm{H}), 0.92(\mathrm{t}, J=8.0 \mathrm{~Hz}, 9 \mathrm{H})$, $0.59(\mathrm{q}, J=8.0 \mathrm{~Hz}, 6 \mathrm{H})$.

${ }^{13} \mathrm{C} \mathrm{NMR} \quad\left(100 \mathrm{MHz}, \mathrm{CDCl}_{3}\right)$

$\delta 151.7,149.9,149.6,124.6,107.3,42.8,36.1,29.9,28.7,21.5,6.8,5.1$.

$\underline{\text { IR }}$

Alpha-Platinum ATR, Bruker, diamond crystal $v=3068,2933,2875,1662,1601,1188 \mathrm{~cm}^{-1}$.

HRMS ESI-TOF

$(\mathrm{M}+\mathrm{H})^{+}$Calcd for $\mathrm{C}_{18} \mathrm{H}_{30} \mathrm{NOSi} 304.2091$; Found 304.2092 

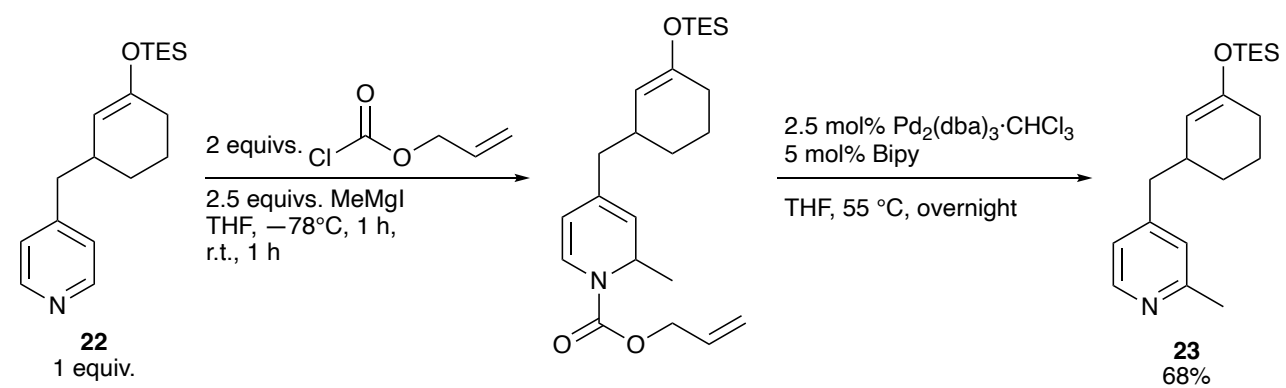

A dry round-bottomed flask was charged with a $0.1 \mathrm{M}$ solution of compund $22(82 \mathrm{mg}, 1.0$ equiv., $0.27 \mathrm{mmol}$ ) in anhydrous THF and the solution was cooled to $-78{ }^{\circ} \mathrm{C}$. Allyl chloroformate $(0.06 \mathrm{~mL}, 2.0$ equivs. $0.54 \mathrm{mmol})$ was added dropwise and the resulting solution was stirred for 1 hour at $-78{ }^{\circ} \mathrm{C}$. MeMgl $(0.68 \mathrm{~mL}, 2.5$ equivs., $0.68 \mathrm{mmol}, 1 \mathrm{M}$ in diethyl ether $)$ was added to the above mixture in a dropwise manner. The resulting mixture was stirred for 1 hour at $-78{ }^{\circ} \mathrm{C}$ and an additional hour at room temperature. The reaction mixture was quenched by dropwise addition of water $(20 \mathrm{~mL})$ and extracted with EtOAc $(3 \times 20 \mathrm{~mL})$. The combined organic layer was washed with brine, dried with $\mathrm{MgSO}_{4}$, filtered through a cotton plug, and concentrated using a rotary evaporator. Crude methylated dihydropyridine product was obtained and used without further purification.

To a dry $25 \mathrm{~mL}$ round-bottomed flask containing $1.5 \mathrm{~mL}$ of anhydrous THF were added $\mathrm{Pd}_{2}(\mathrm{dba})_{3} \cdot \mathrm{CHCl}_{3}(7 \mathrm{mg}, 2.5 \mathrm{~mol} \%, 0.00675 \mathrm{mmol})$ and 2,2'-bipyridine $(2.1 \mathrm{mg}, 5 \mathrm{~mol} \%, 0.0135$ $\mathrm{mmol}$ ). The resulting mixture was stirred for $30 \mathrm{~min}$. A $0.1 \mathrm{M}$ solution of methylated dihydropyridine product in anhydrous THF was prepared and added dropwise to the above palladium catalyst solution. The resulting mixture was stirred and heated at reflux overnight. The mixture was cooled to ambient temperature and concentrated using a rotary evaporator. The crude product was purified by flash column chromatography using EtOAc/hexanes to afford product $\mathbf{2 3}$ as colorless oil (58 mg, $68 \%$ yield).

Chromatography: $30 \%$ EtOAc in hexanes. $\left(R_{f}=0.25\right)$

1 NMR $\quad\left(400 \mathrm{MHz}, \mathrm{CDCl}_{3}\right)$

$\delta 8.36(\mathrm{~d}, J=5.1 \mathrm{~Hz}, 1 \mathrm{H}), 6.95(\mathrm{~s}, 1 \mathrm{H}), 6.89(\mathrm{~d}, J=5.1 \mathrm{~Hz}, 1 \mathrm{H}), 4.69(\mathrm{~s}, 1 \mathrm{H}), 2.51$ (s, 3H), $2.51-2.48(\mathrm{~m}, 2 \mathrm{H}), 2.47-2.42(\mathrm{~m}, 1 \mathrm{H}), 2.08-1.92(\mathrm{~m}, 2 \mathrm{H}), 1,81-1.72$ $(\mathrm{m}, 1 \mathrm{H}), 1.68-1.59(\mathrm{~m}, 1 \mathrm{H}), 1.59-1.48(\mathrm{~m}, 1 \mathrm{H}), 1.18-1.08(\mathrm{~m}, 1 \mathrm{H}), 0.95(\mathrm{t}, J=$ $8.0 \mathrm{~Hz}, 9 \mathrm{H}), 0.62(\mathrm{q}, \mathrm{J}=8.0 \mathrm{~Hz}, 6 \mathrm{H})$.

${ }^{13} \mathrm{C} \mathrm{NMR} \quad\left(100 \mathrm{MHz}, \mathrm{CDCl}_{3}\right)$

$\delta 158.2,151.7,150.2,149.0,124.3,121.8,107.5,42.9,36.2,30.0,28.9,24.5,21.6$, $6.8,5.2$.

IR Alpha-Platinum ATR, Bruker, diamond crystal $v=3010.2952 .2874,1663,1604,1190 \mathrm{~cm}^{-1}$.

HRMS ESI-TOF

$(\mathrm{M}+\mathrm{H})^{+}$Calcd for $\mathrm{C}_{19} \mathrm{H}_{32} \mathrm{NOSi} 318.2248$; Found 318.2247. 
${ }^{1} \mathrm{H}-,{ }^{13} \mathrm{C}-\mathrm{NMR}$ and $2 \mathrm{D}$ spectra:

${ }^{1} \mathrm{H}$ NMR (400 MHz, $\left.\mathrm{CDCl}_{3}\right)$ and ${ }^{13} \mathrm{C}$ NMR (100 MHz, $\left.\mathrm{CDCl}_{3}\right)$ for 9:
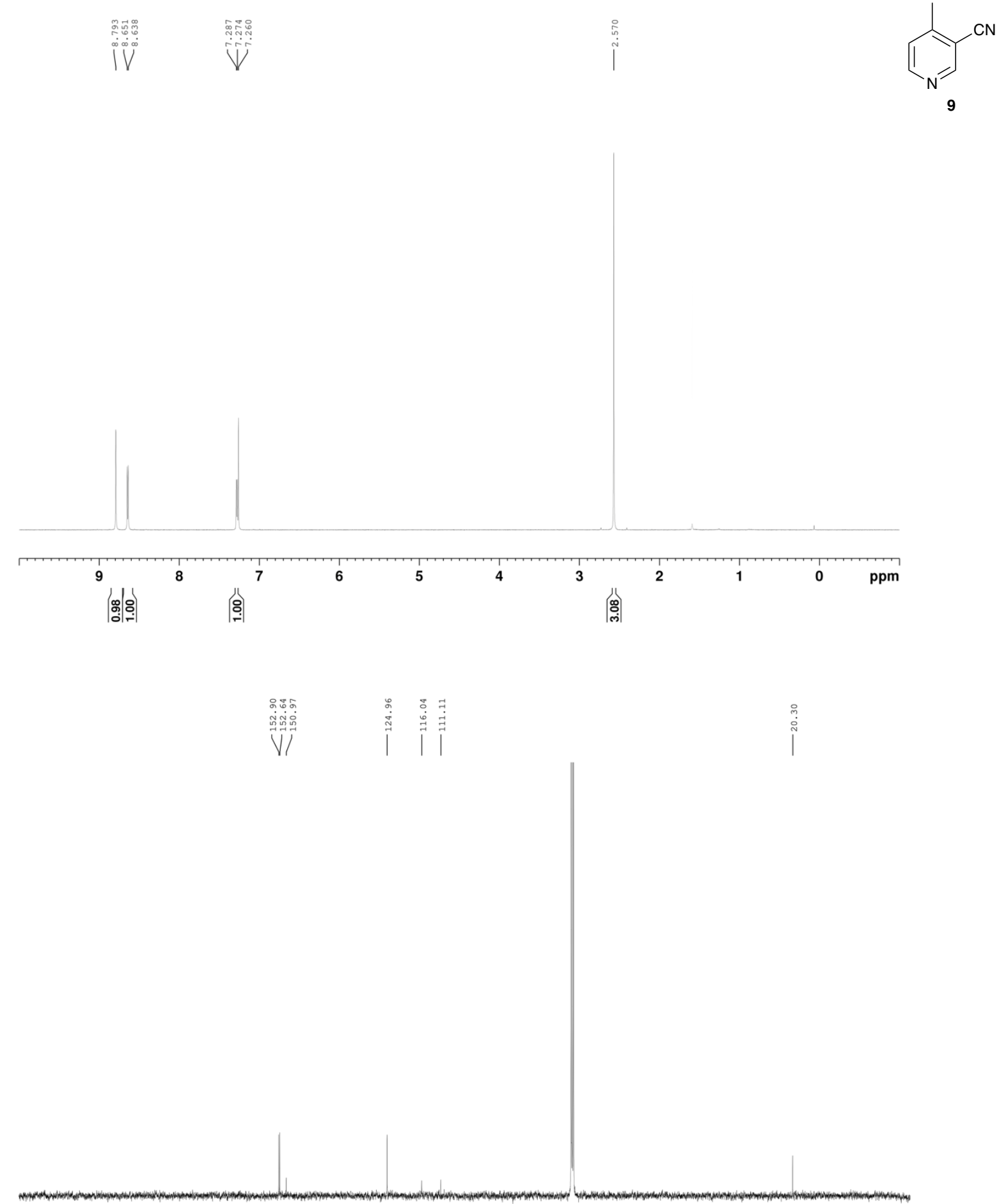

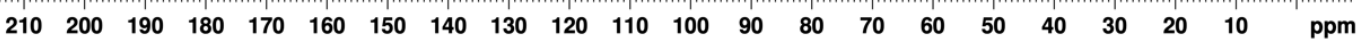


${ }^{1} \mathrm{H} \mathrm{NMR}\left(400 \mathrm{MHz}, \mathrm{CDCl}_{3}\right)$ and $\left.{ }^{13} \mathrm{C} \mathrm{NMR} \mathrm{(100} \mathrm{MHz,} \mathrm{CDCl}_{3}\right)$ for 10:
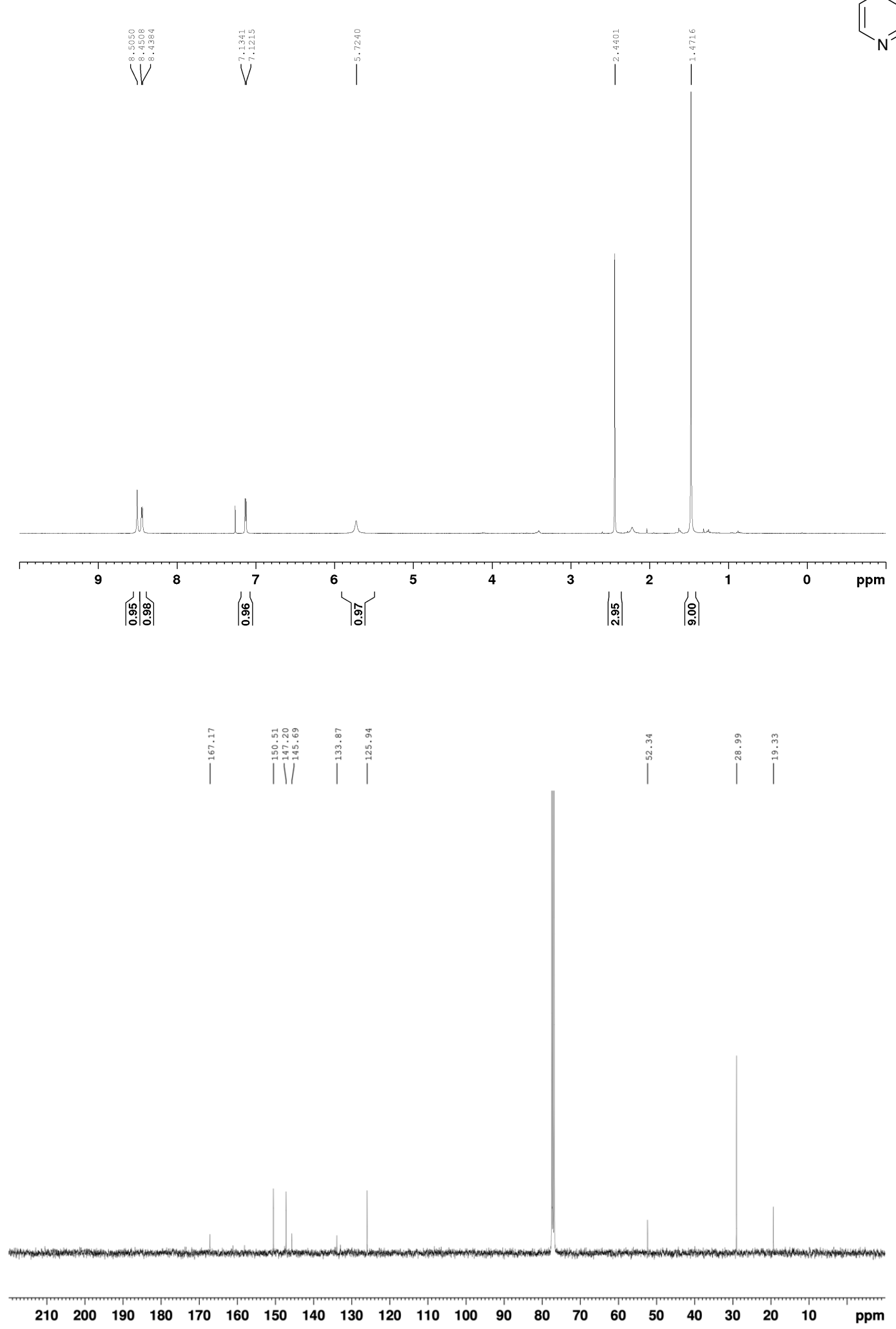
${ }^{1} \mathrm{H} \mathrm{NMR}\left(400 \mathrm{MHz}, \mathrm{CDCl}_{3}\right)$ and ${ }^{13} \mathrm{C} \mathrm{NMR}\left(100 \mathrm{MHz}, \mathrm{CDCl}_{3}\right)$ for 11:
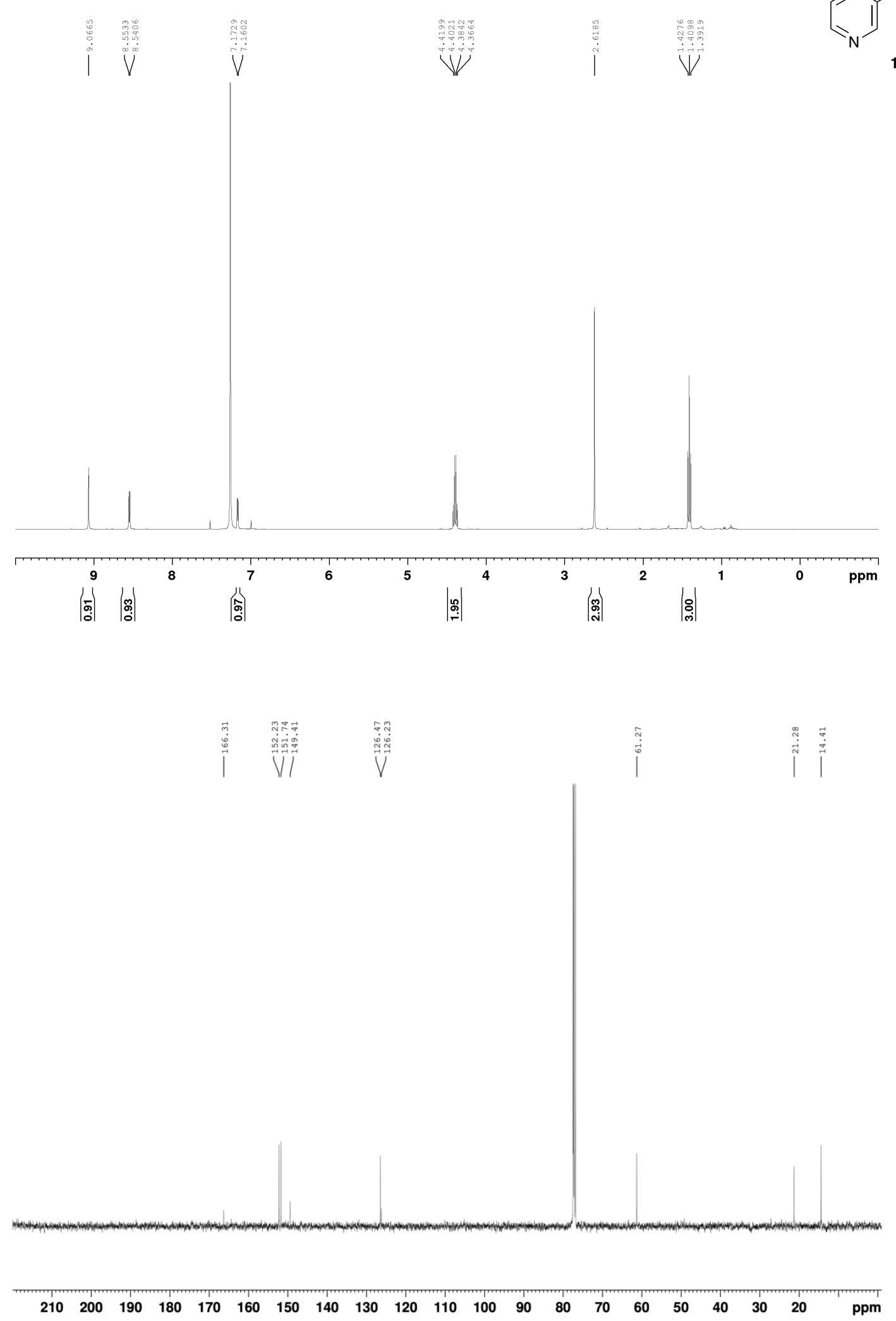
${ }^{1} \mathrm{H} \mathrm{NMR}\left(400 \mathrm{MHz}, \mathrm{CDCl}_{3}\right)$ and ${ }^{13} \mathrm{C} \mathrm{NMR}\left(100 \mathrm{MHz}, \mathrm{CDCl}_{3}\right)$ for 12:
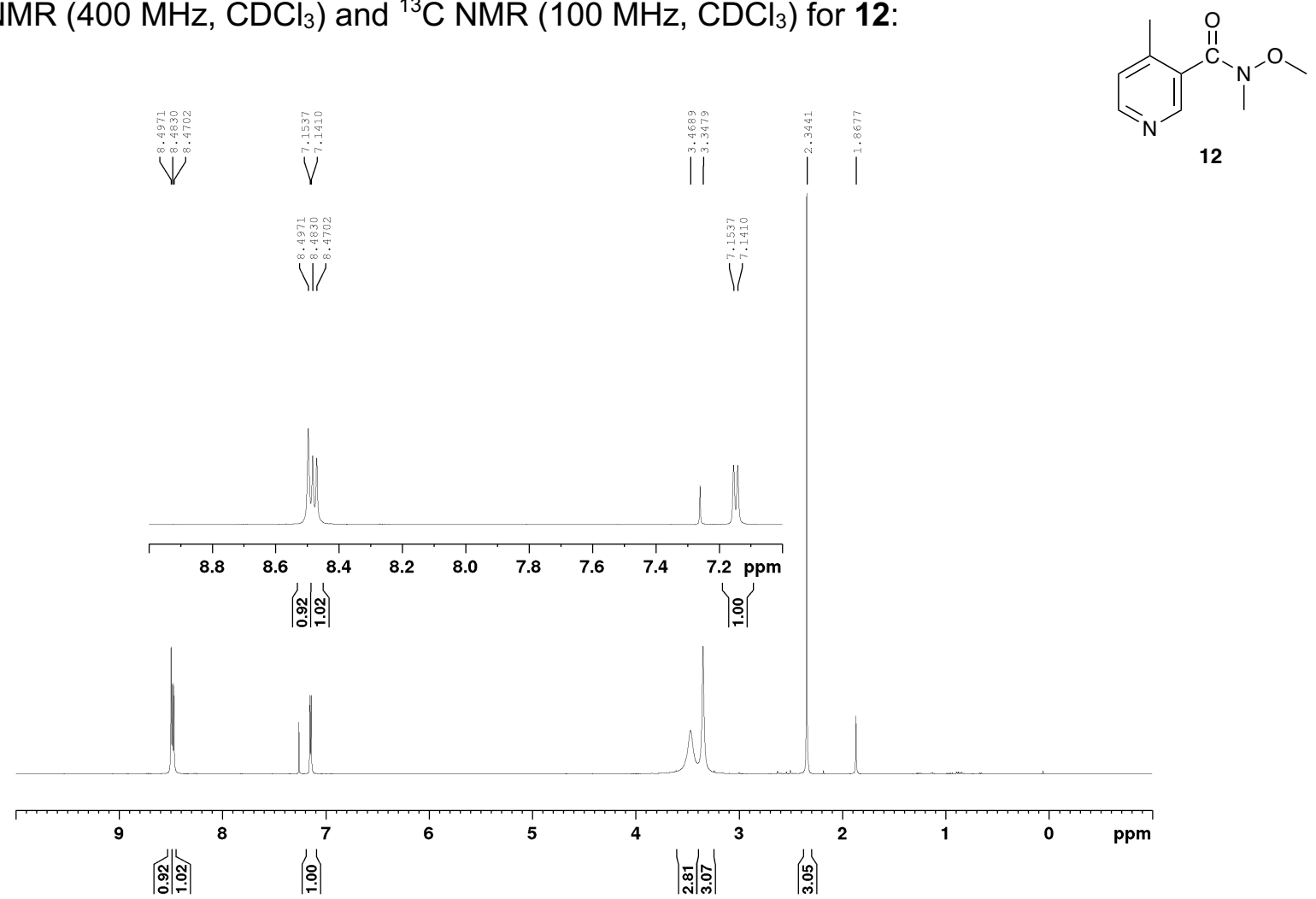

12

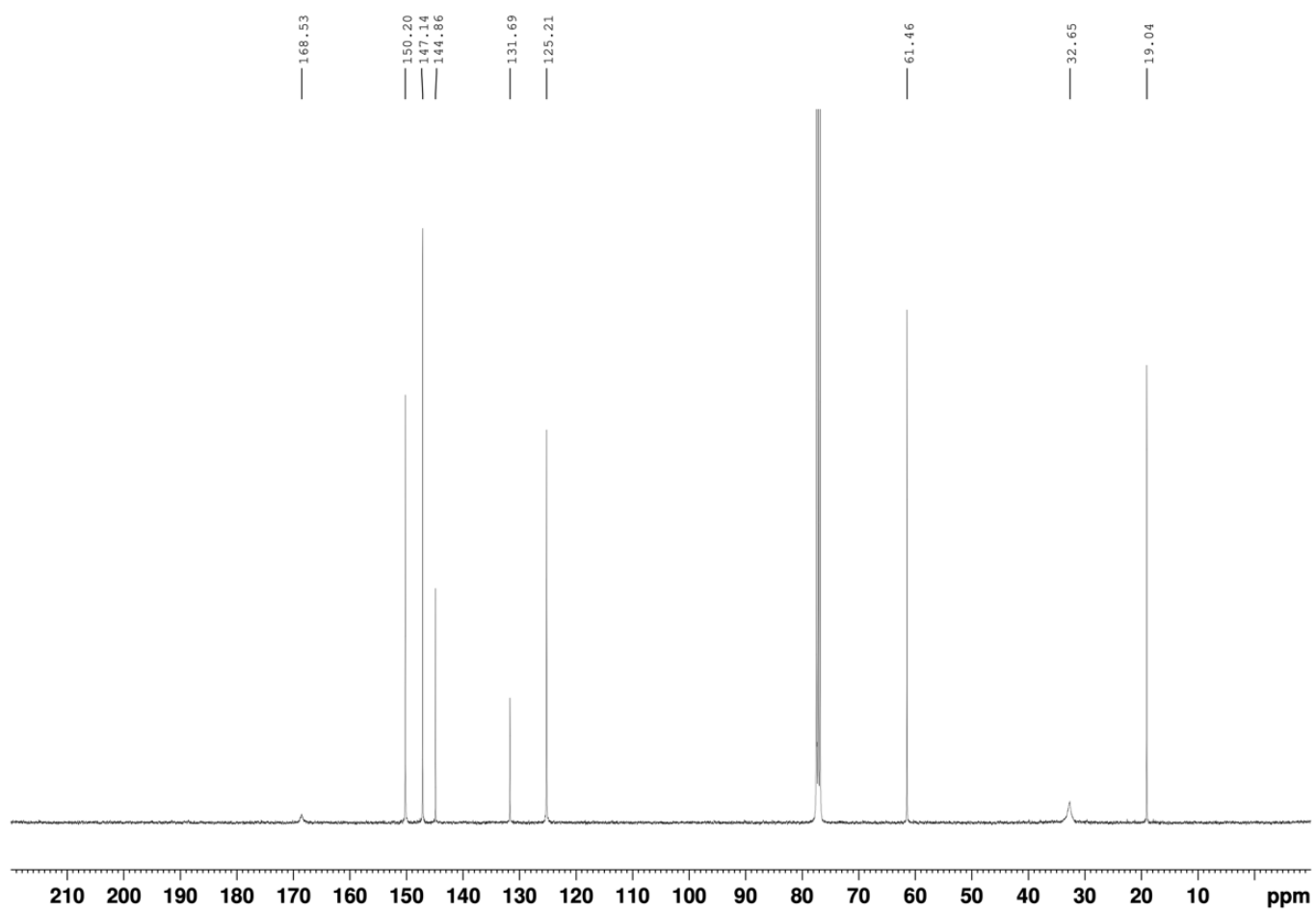


${ }^{1} \mathrm{H} \mathrm{NMR}\left(400 \mathrm{MHz}, \mathrm{CDCl}_{3}\right)$ and ${ }^{13} \mathrm{C} \mathrm{NMR}\left(100 \mathrm{MHz}, \mathrm{CDCl}_{3}\right)$ for 13 :
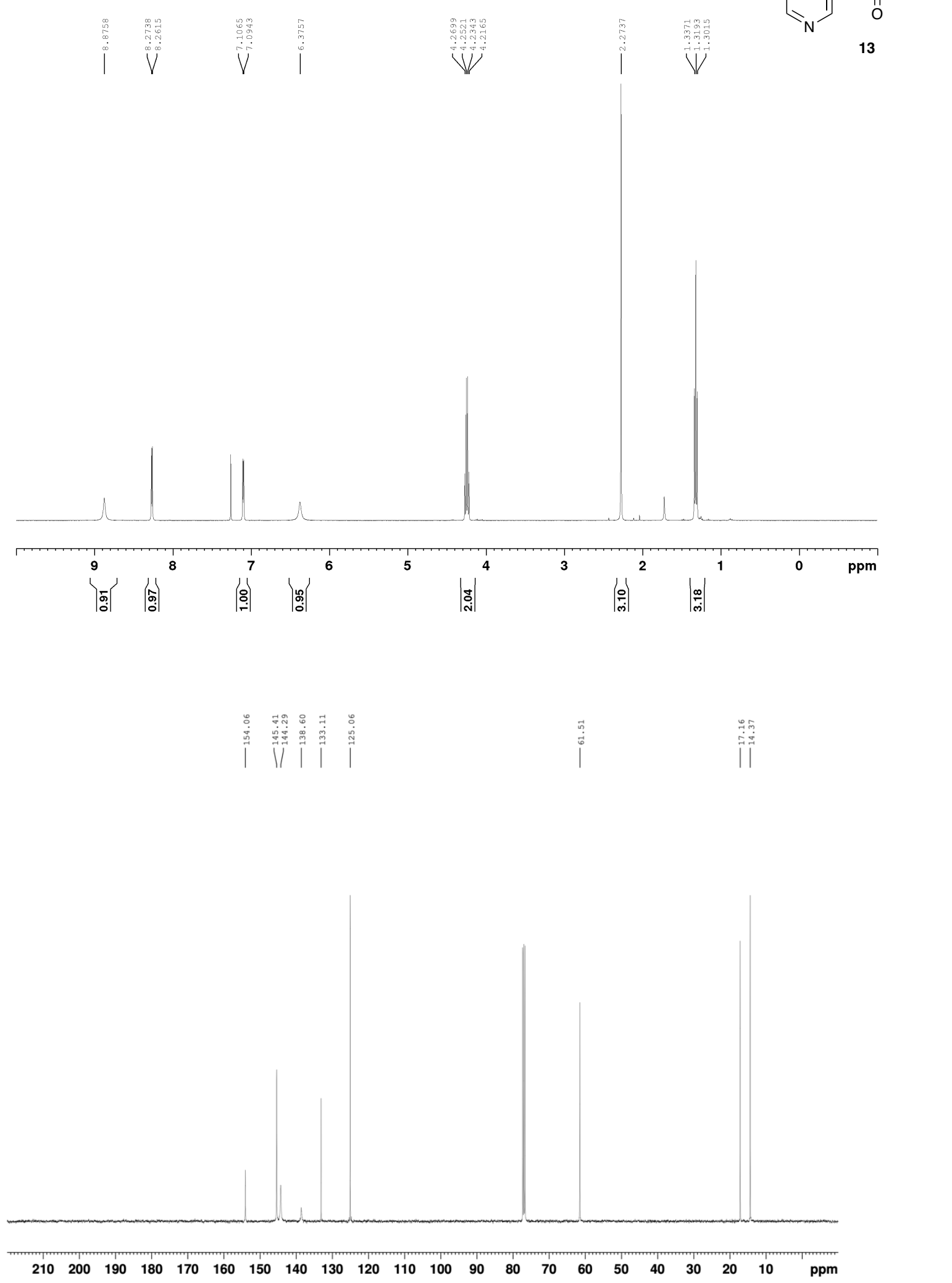
${ }^{1} \mathrm{H}$ NMR (400 MHz, $\left.\mathrm{CDCl}_{3}\right)$ and ${ }^{13} \mathrm{C}$ NMR (100 MHz, $\left.\mathrm{CDCl}_{3}\right)$ for 14:
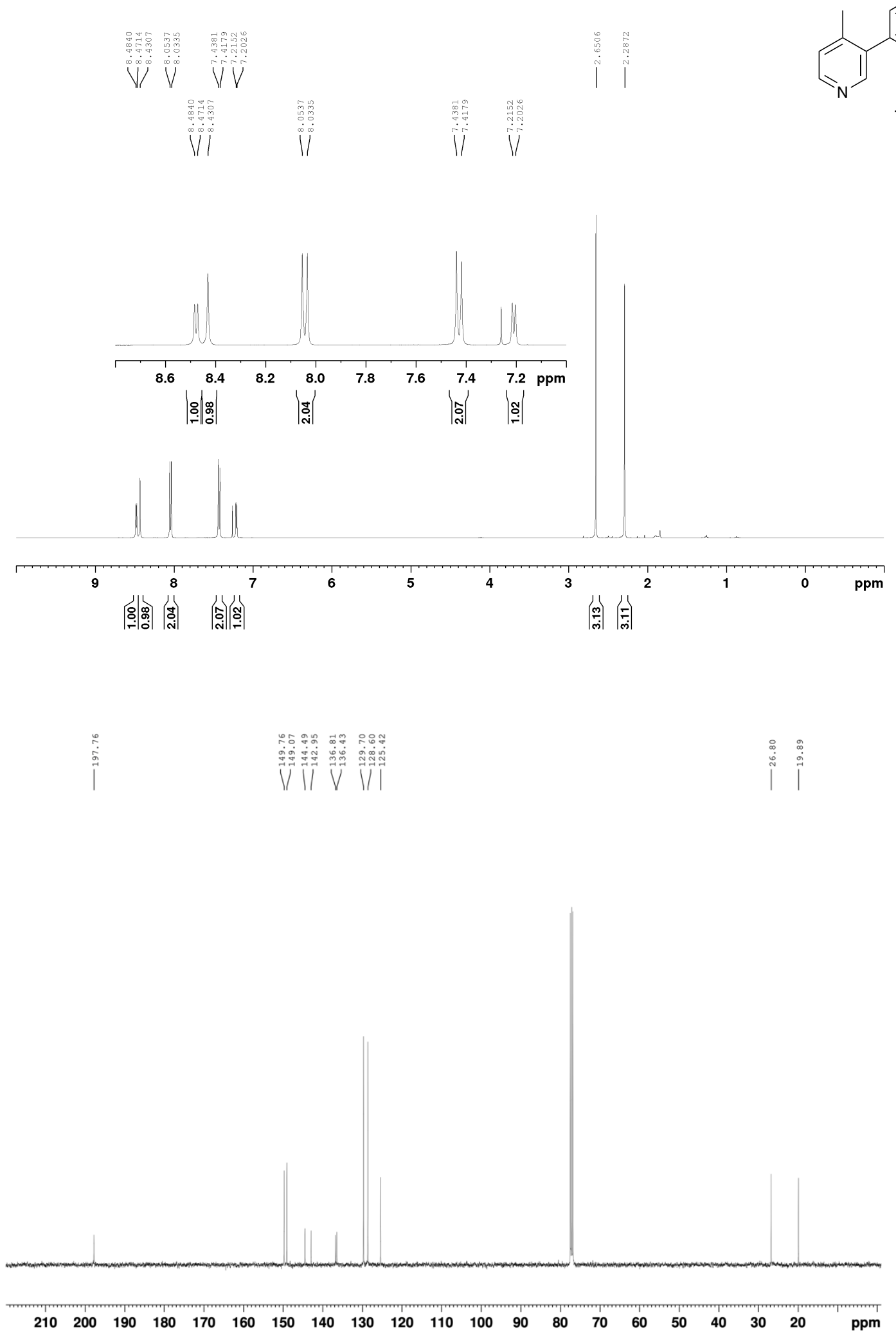
${ }^{1} \mathrm{H}$ NMR $\left(400 \mathrm{MHz}, \mathrm{CDCl}_{3}\right)$ and ${ }^{13} \mathrm{C}$ NMR $\left(100 \mathrm{MHz}, \mathrm{CDCl}_{3}\right)$ for $15:$
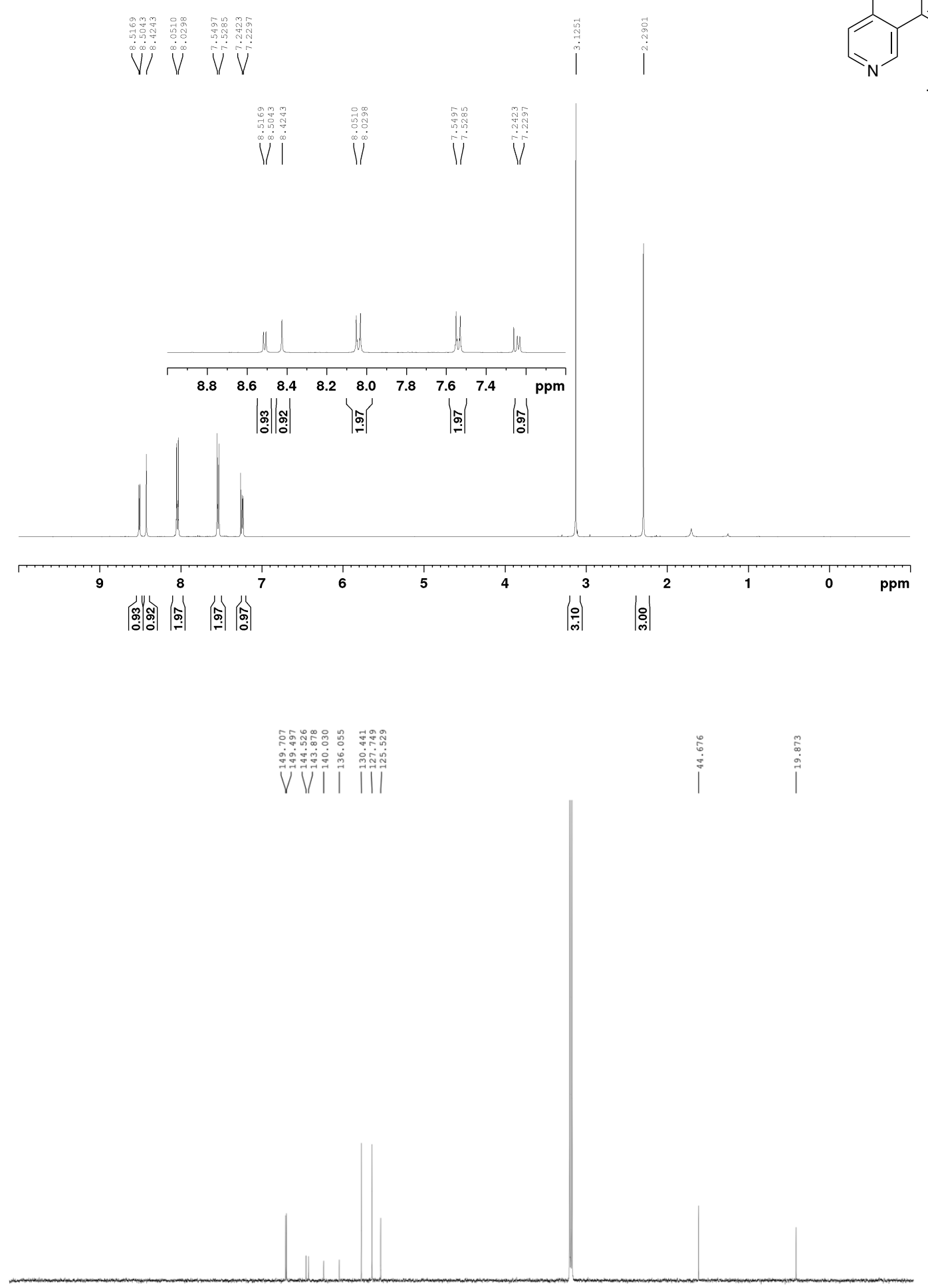

$\begin{array}{llllllllllllllllllllll}210 & 200 & 190 & 180 & 170 & 160 & 150 & 140 & 130 & 120 & 110 & 100 & 90 & 80 & 70 & 60 & 50 & 40 & 30 & 20 & 10 & \mathrm{ppm}\end{array}$ 
${ }^{1} \mathrm{H}$ NMR $\left(400 \mathrm{MHz}, \mathrm{CDCl}_{3}\right)$ and ${ }^{13} \mathrm{C}$ NMR (100 MHz, $\left.\mathrm{CDCl}_{3}\right)$ for 16:

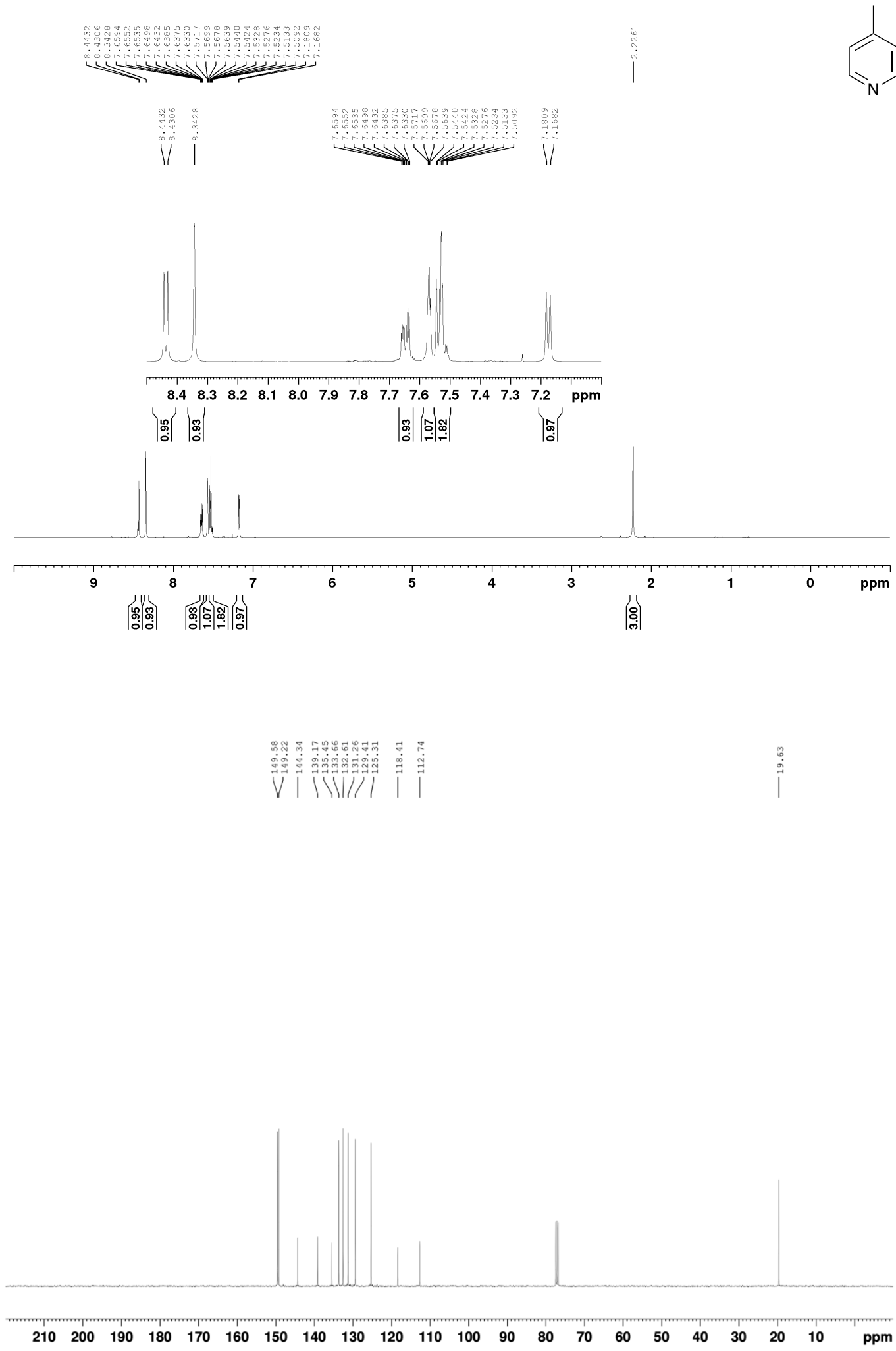


${ }^{1} \mathrm{H} \mathrm{NMR}\left(400 \mathrm{MHz}, \mathrm{CDCl}_{3}\right)$ and ${ }^{13} \mathrm{C} \mathrm{NMR}\left(100 \mathrm{MHz}, \mathrm{CDCl}_{3}\right)$ for 17 :

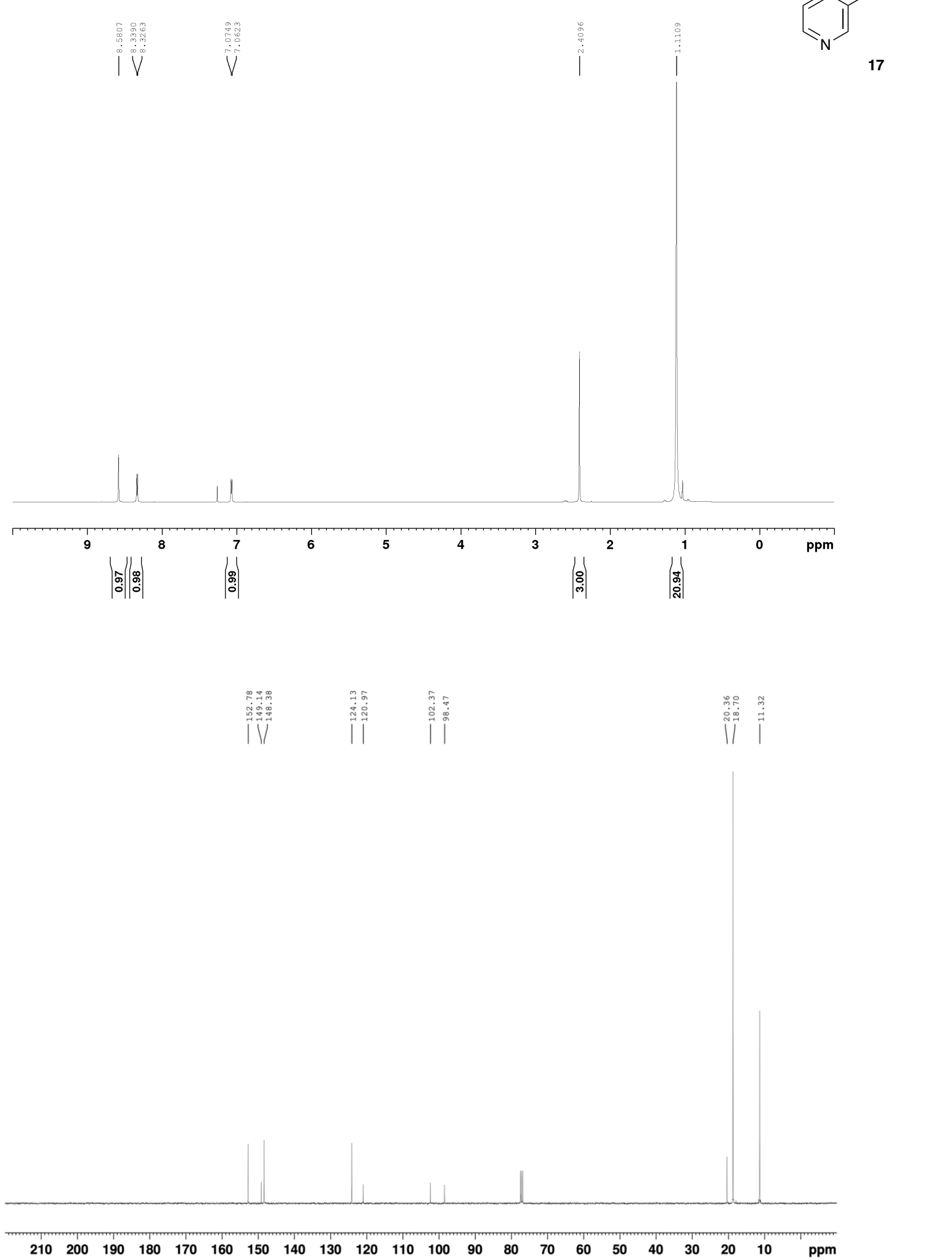


${ }^{1} \mathrm{H}$ NMR $\left(400 \mathrm{MHz}, \mathrm{CDCl}_{3}\right)$ and ${ }^{13} \mathrm{C}$ NMR $\left(100 \mathrm{MHz}, \mathrm{CDCl}_{3}\right)$ for 18:
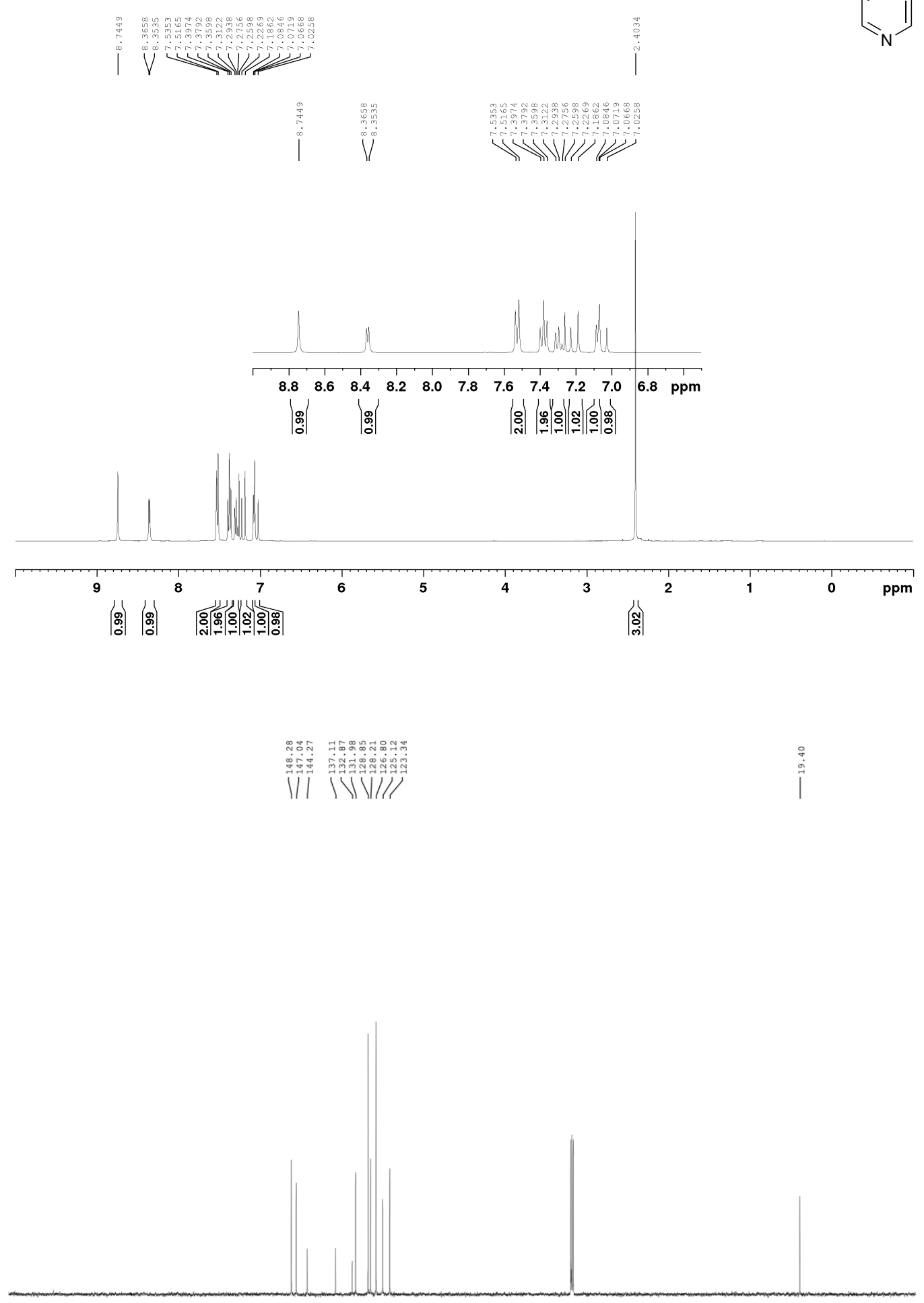

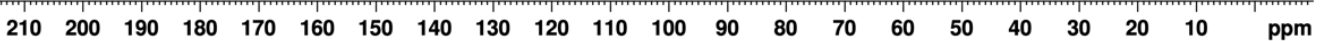


${ }^{1} \mathrm{H}$ NMR $\left(300 \mathrm{MHz}, \mathrm{CDCl}_{3}\right)$ and ${ }^{13} \mathrm{C} \mathrm{NMR}\left(75 \mathrm{MHz}, \mathrm{CDCl}_{3}\right)$ for $\mathbf{F}$ :

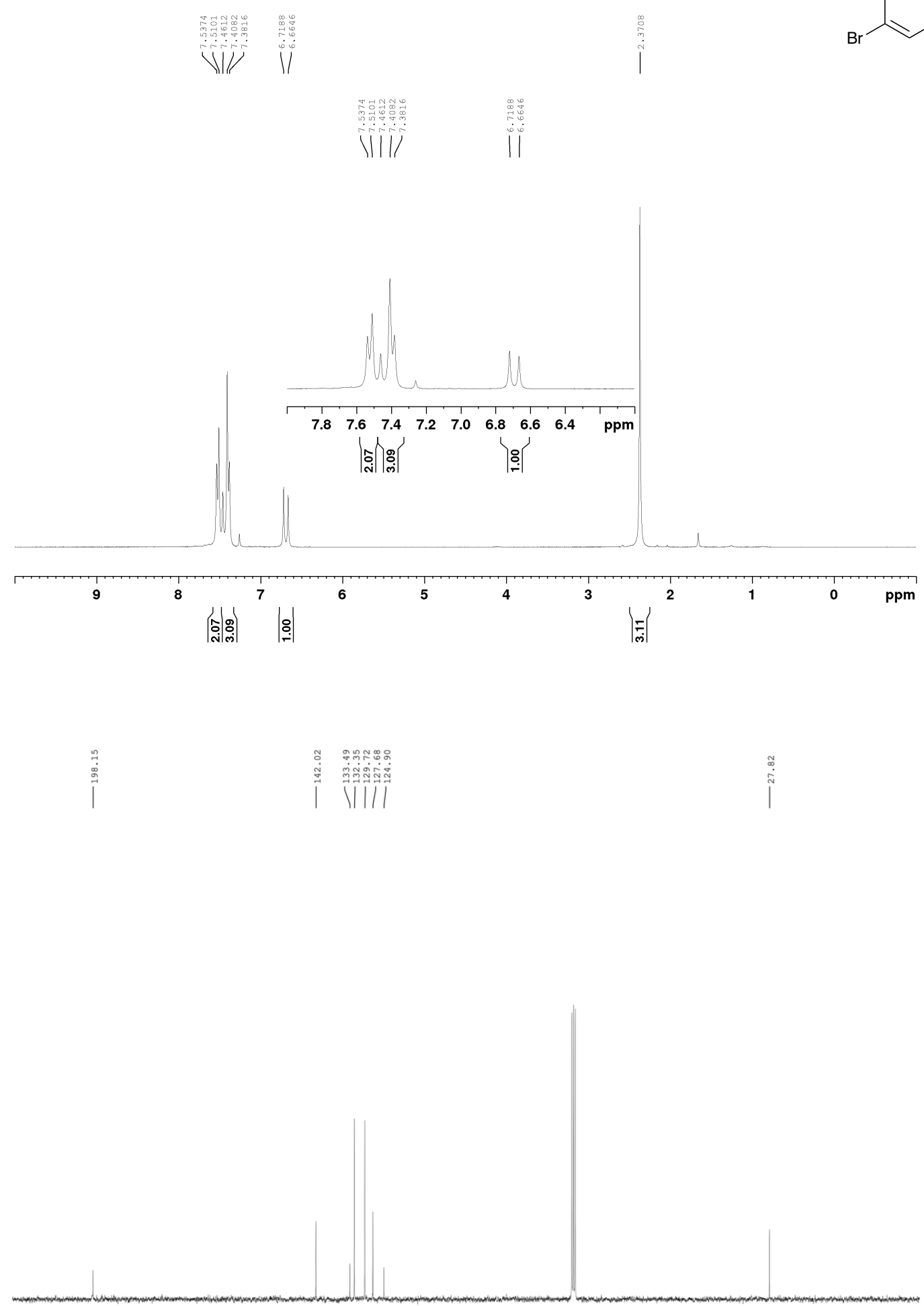

$\begin{array}{llllllllllllllllllllll}210 & 200 & 190 & 180 & 170 & 160 & 150 & 140 & 130 & 120 & 110 & 100 & 90 & 80 & 70 & 60 & 50 & 40 & 30 & 20 & 10 & \mathrm{ppm}\end{array}$ 
${ }^{1} \mathrm{H}$ NMR $\left(300 \mathrm{MHz}, \mathrm{CDCl}_{3}\right)$ and ${ }^{13} \mathrm{C} \mathrm{NMR}\left(75 \mathrm{MHz}, \mathrm{CDCl}_{3}\right)$ for 1A:

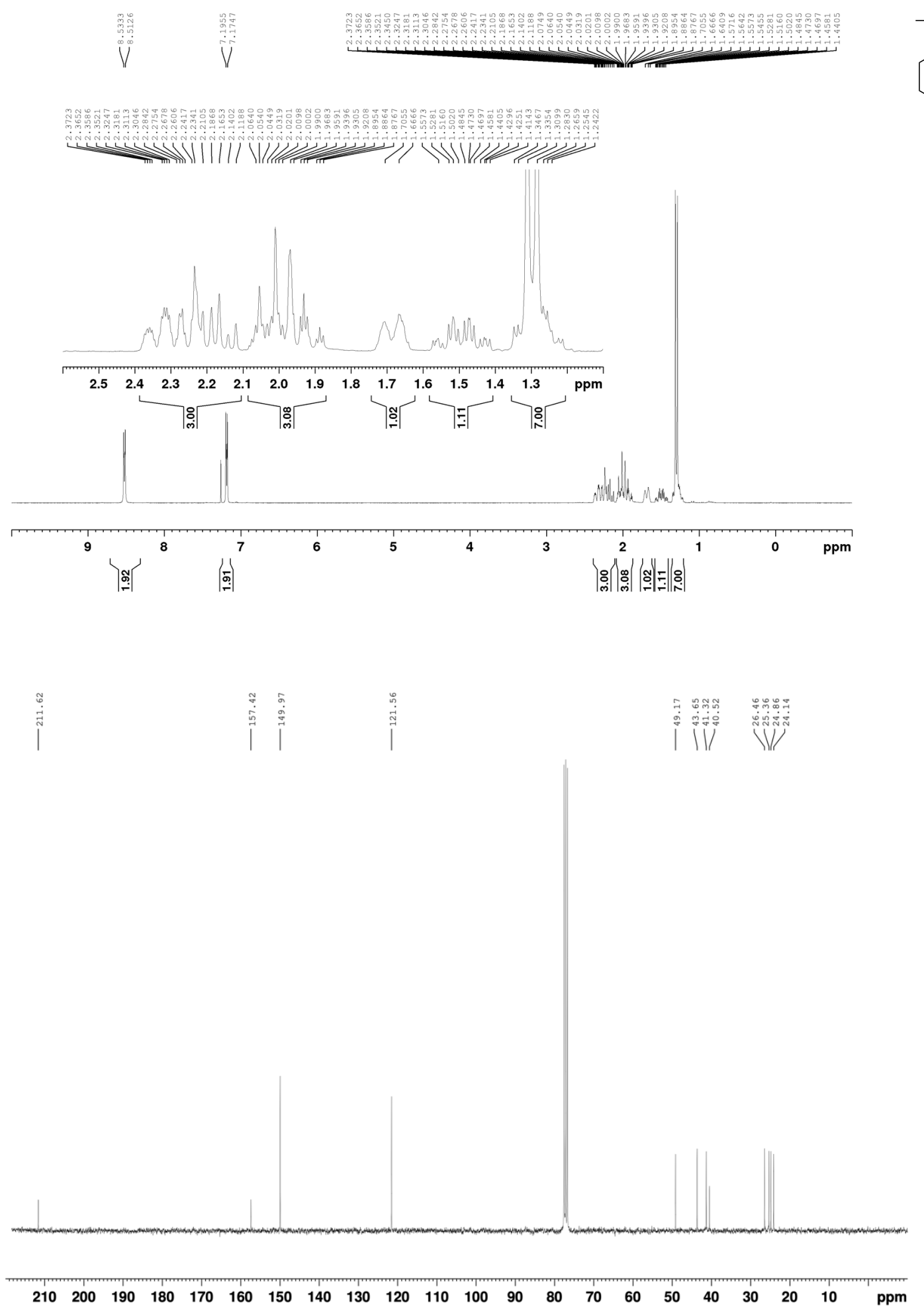


${ }^{1} \mathrm{H} \mathrm{NMR}\left(400 \mathrm{MHz}, \mathrm{CDCl}_{3}\right)$ and ${ }^{13} \mathrm{C} \mathrm{NMR}\left(100 \mathrm{MHz}, \mathrm{CDCl}_{3}\right)$ for $\mathbf{2 A}$ :
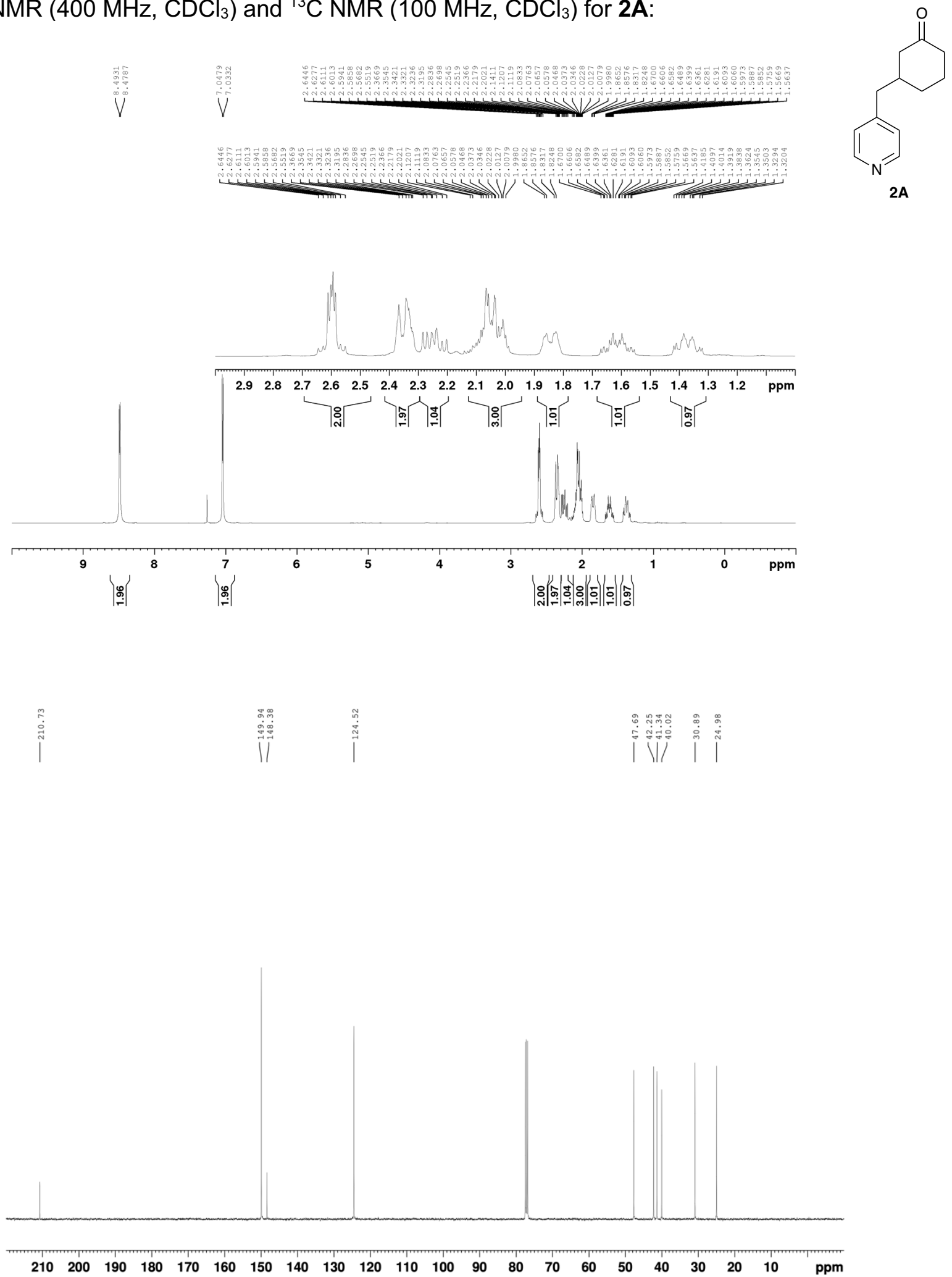
${ }^{1} \mathrm{H} \mathrm{NMR}\left(400 \mathrm{MHz}, \mathrm{CDCl}_{3}\right)$ and ${ }^{13} \mathrm{C} \mathrm{NMR}\left(100 \mathrm{MHz}, \mathrm{CDCl}_{3}\right)$ for 3A-1:

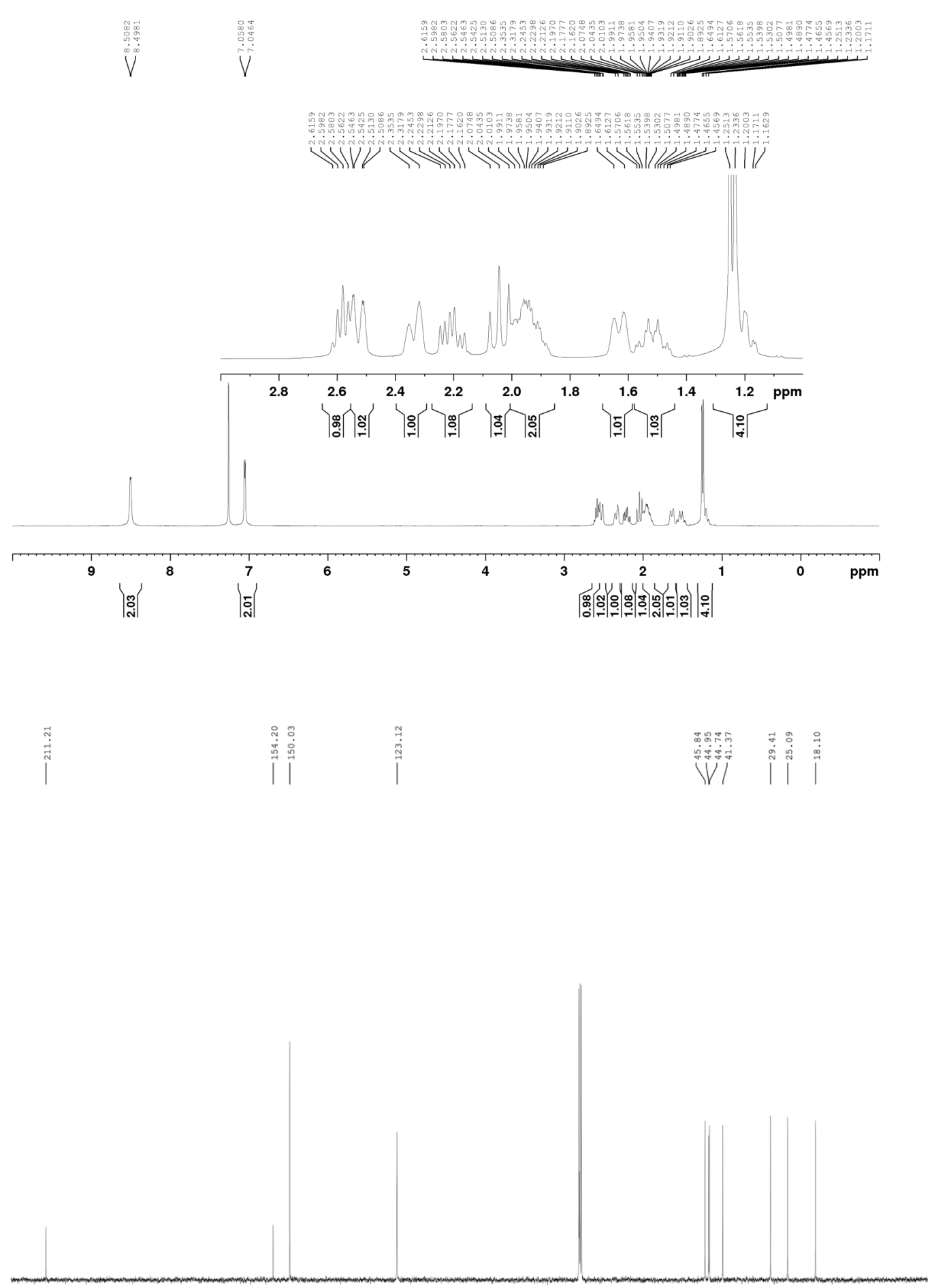


${ }^{1} \mathrm{H} \mathrm{NMR}\left(400 \mathrm{MHz}, \mathrm{CDCl}_{3}\right)$ and ${ }^{13} \mathrm{C} \mathrm{NMR}\left(100 \mathrm{MHz}, \mathrm{CDCl}_{3}\right)$ for 3A-2:
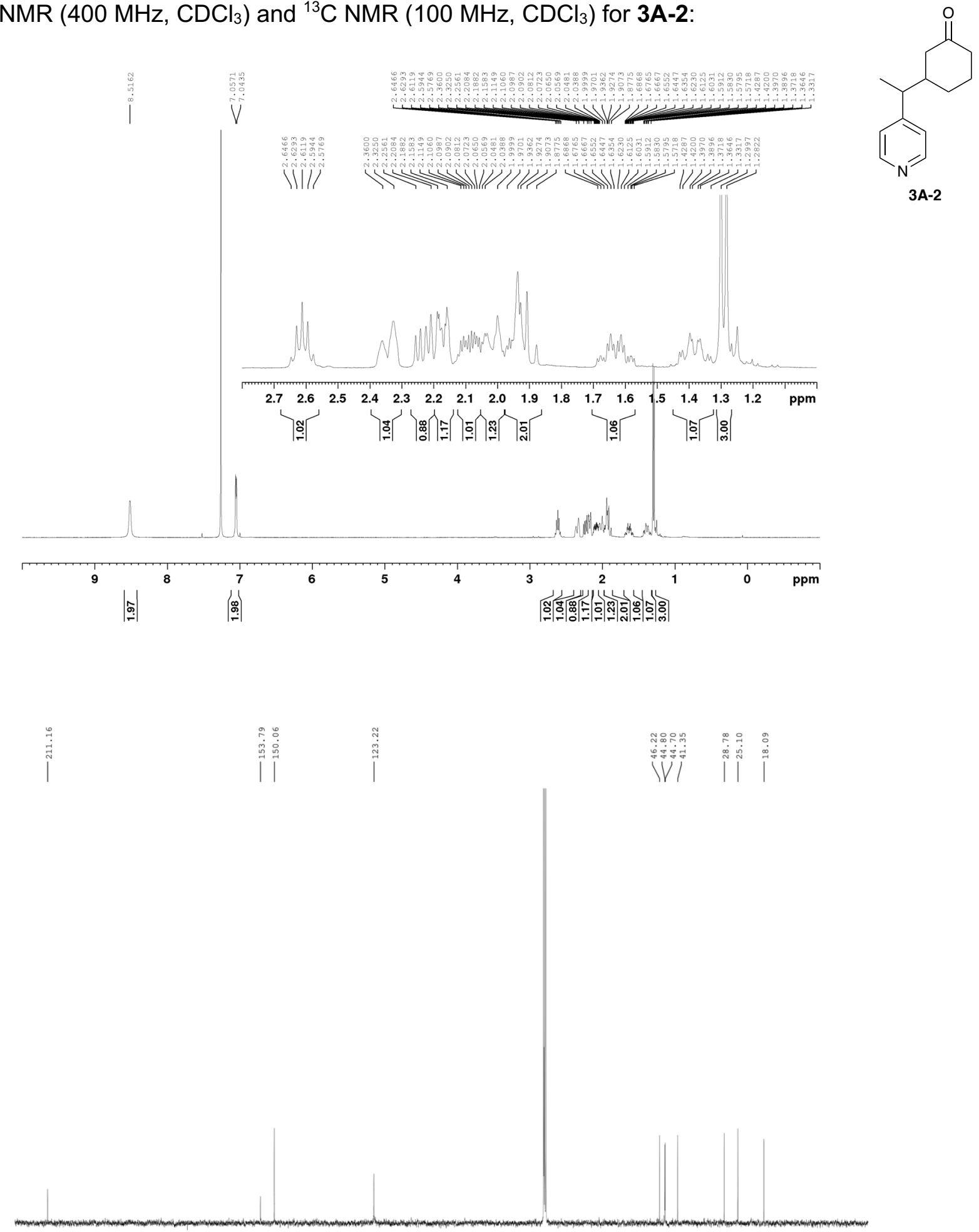

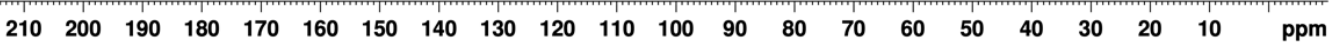


${ }^{1} \mathrm{H} \mathrm{NMR}\left(400 \mathrm{MHz}, \mathrm{CDCl}_{3}\right)$ and ${ }^{13} \mathrm{C} \mathrm{NMR}\left(100 \mathrm{MHz}, \mathrm{CDCl}_{3}\right)$ for 3B-1:
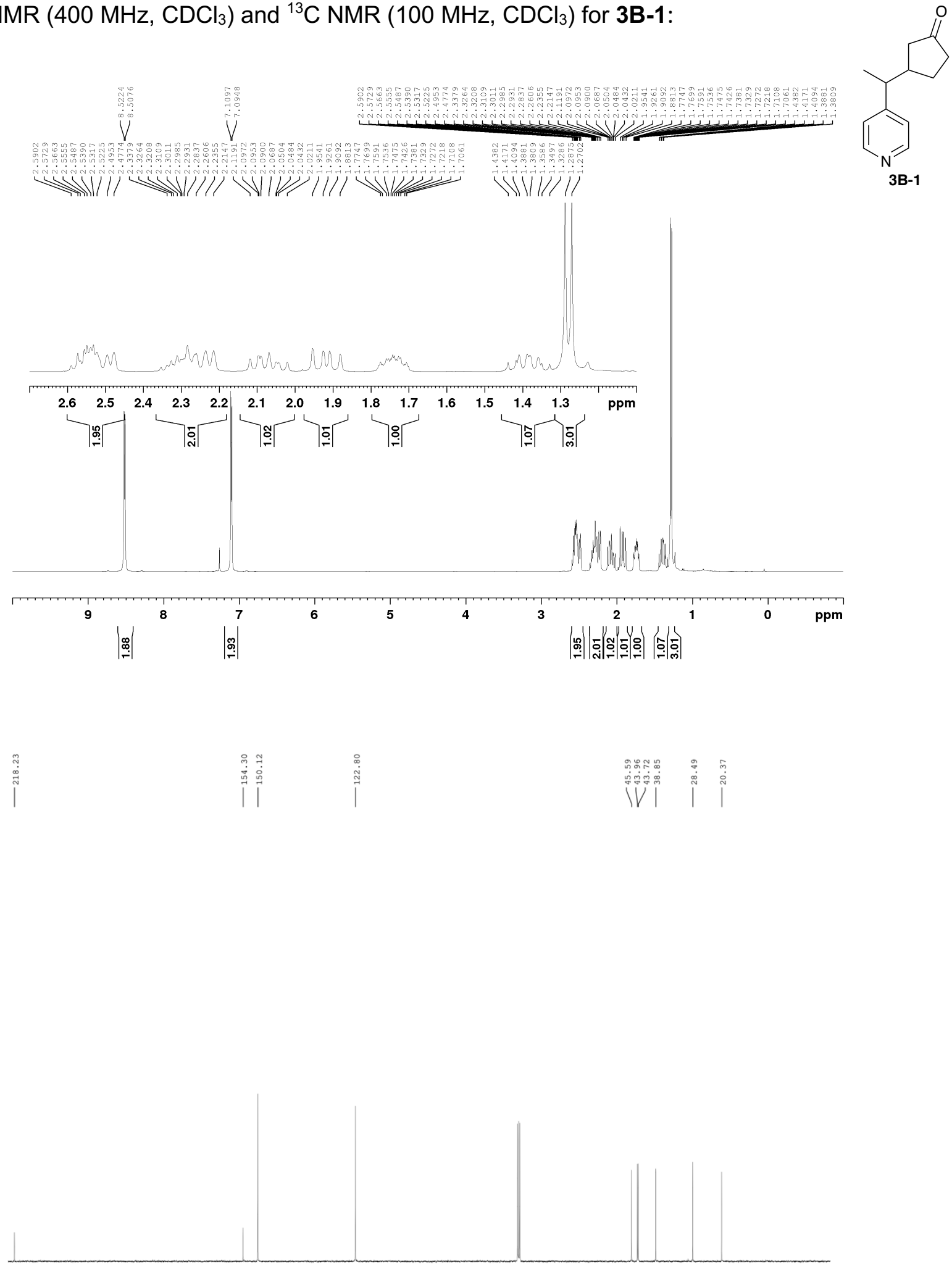

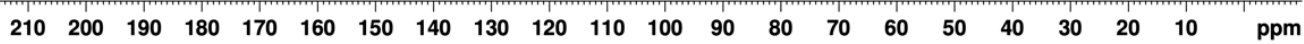

${ }^{1} \mathrm{H} \mathrm{NMR}\left(400 \mathrm{MHz}, \mathrm{CDCl}_{3}\right)$ and ${ }^{13} \mathrm{C} \mathrm{NMR}\left(100 \mathrm{MHz}, \mathrm{CDCl}_{3}\right)$ for 3B-2:

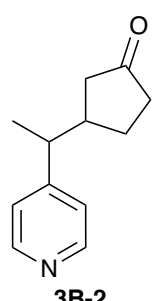




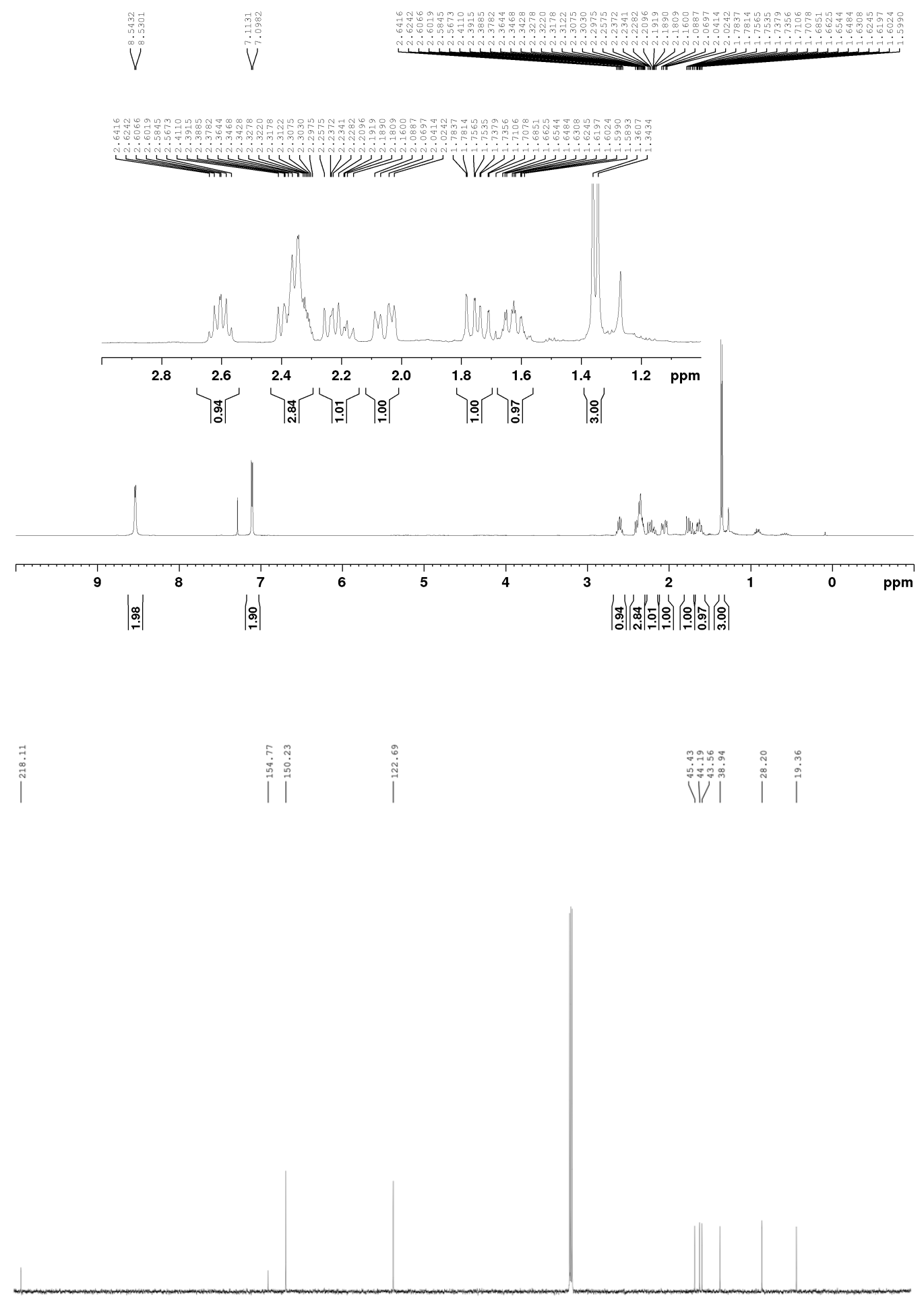

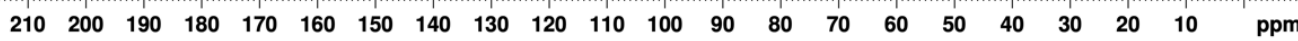


${ }^{1} \mathrm{H}$ NMR $\left(400 \mathrm{MHz}, \mathrm{CDCl}_{3}\right)$ and ${ }^{13} \mathrm{C} \mathrm{NMR}\left(100 \mathrm{MHz}, \mathrm{CDCl}_{3}\right)$ for 4A-1:
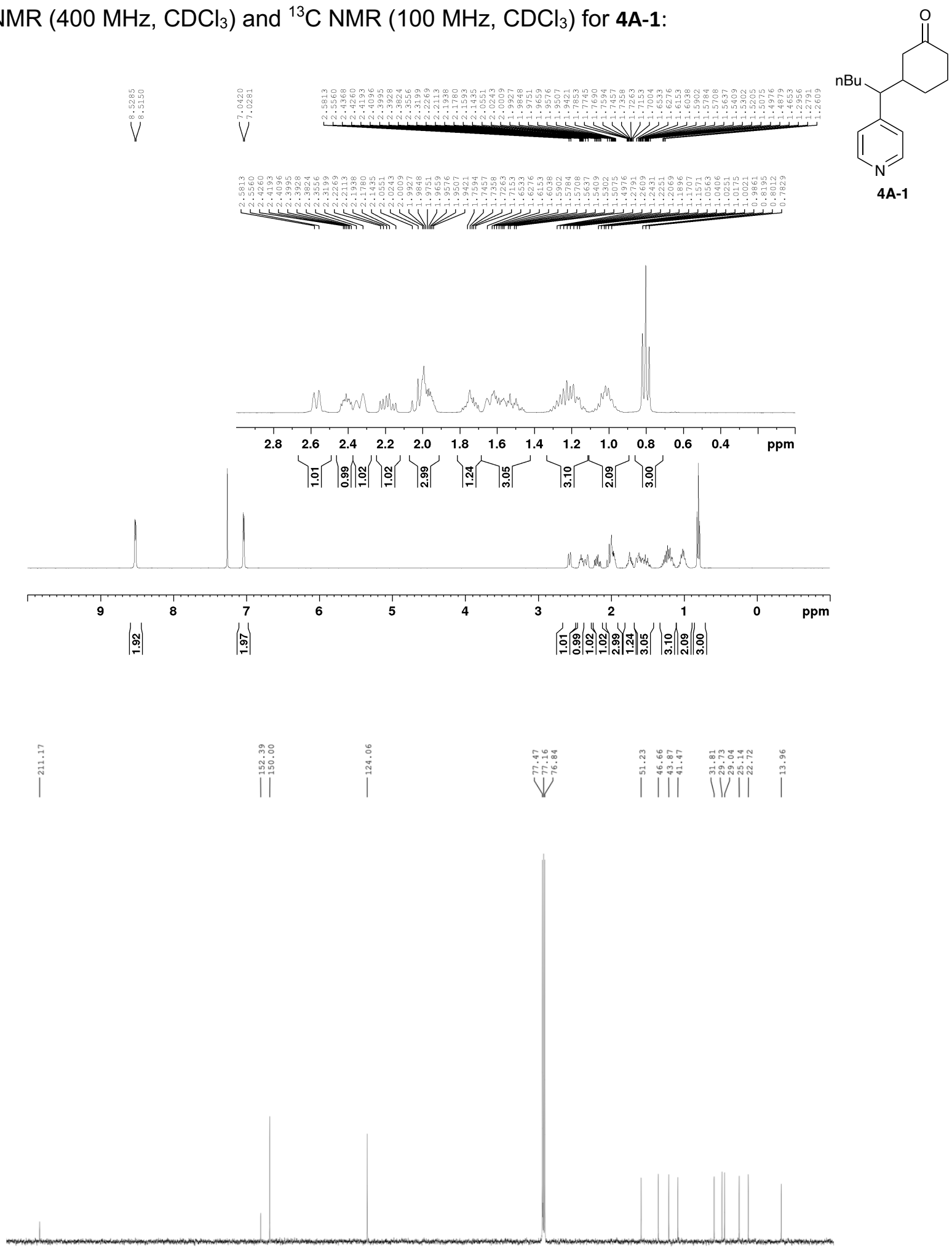

$\begin{array}{lllllllllllllllllllll}210 & 200 & 190 & 180 & 170 & 160 & 150 & 140 & 130 & 120 & 110 & 100 & 90 & 80 & 70 & 60 & 50 & 40 & 30 & 20 & \mathrm{ppm}\end{array}$ 
${ }^{1} \mathrm{H} \mathrm{NMR}\left(400 \mathrm{MHz}, \mathrm{CDCl}_{3}\right)$ and ${ }^{13} \mathrm{C} \mathrm{NMR}\left(100 \mathrm{MHz}, \mathrm{CDCl}_{3}\right)$ for 4A-2:
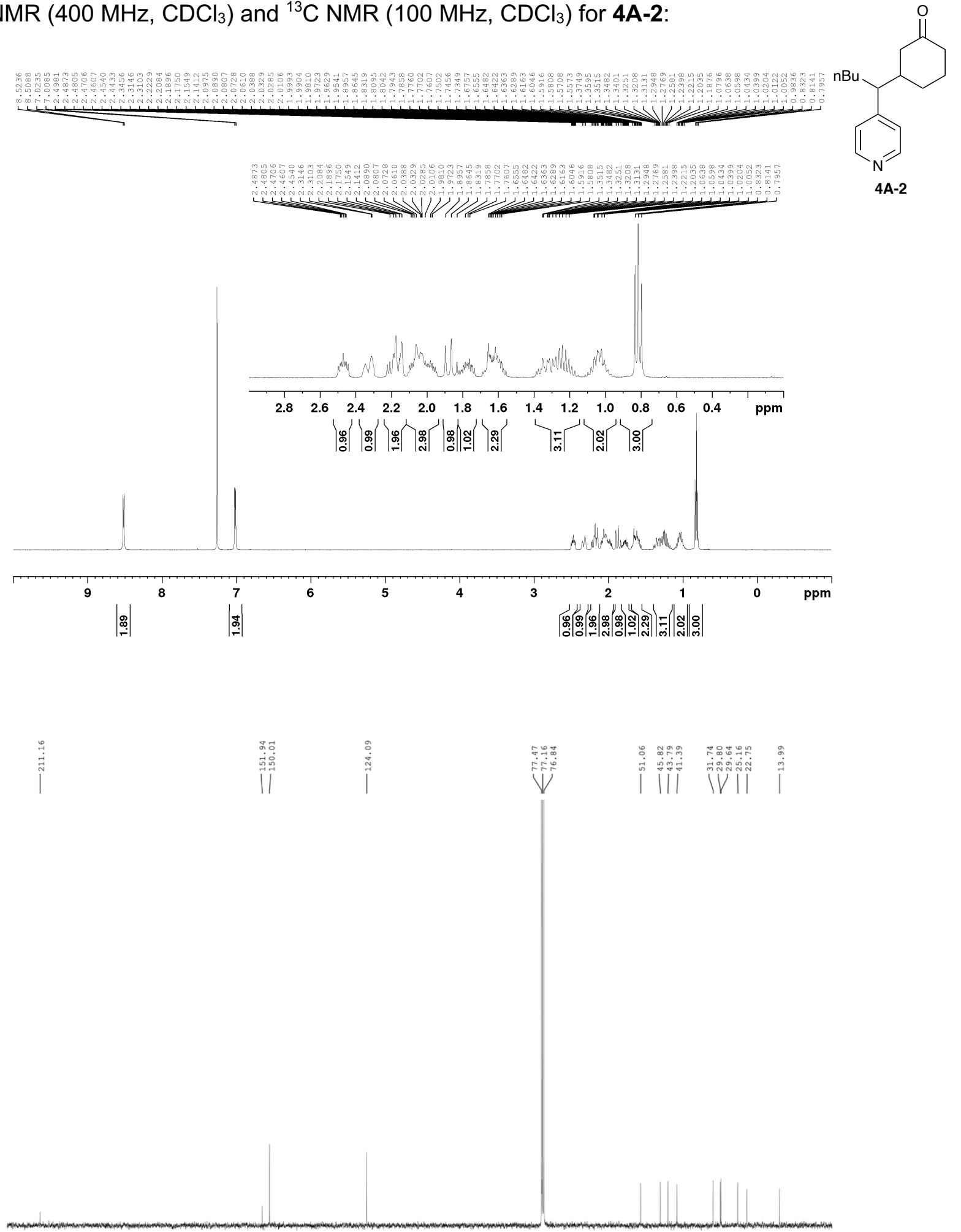

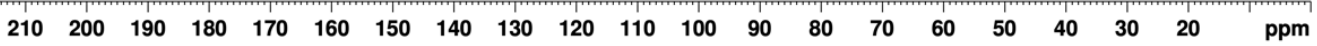


${ }^{1} \mathrm{H} \mathrm{NMR}\left(400 \mathrm{MHz}, \mathrm{CDCl}_{3}\right)$ and ${ }^{13} \mathrm{C} \mathrm{NMR}\left(100 \mathrm{MHz}, \mathrm{CDCl}_{3}\right)$ for $\mathbf{5 A - 1}$ :
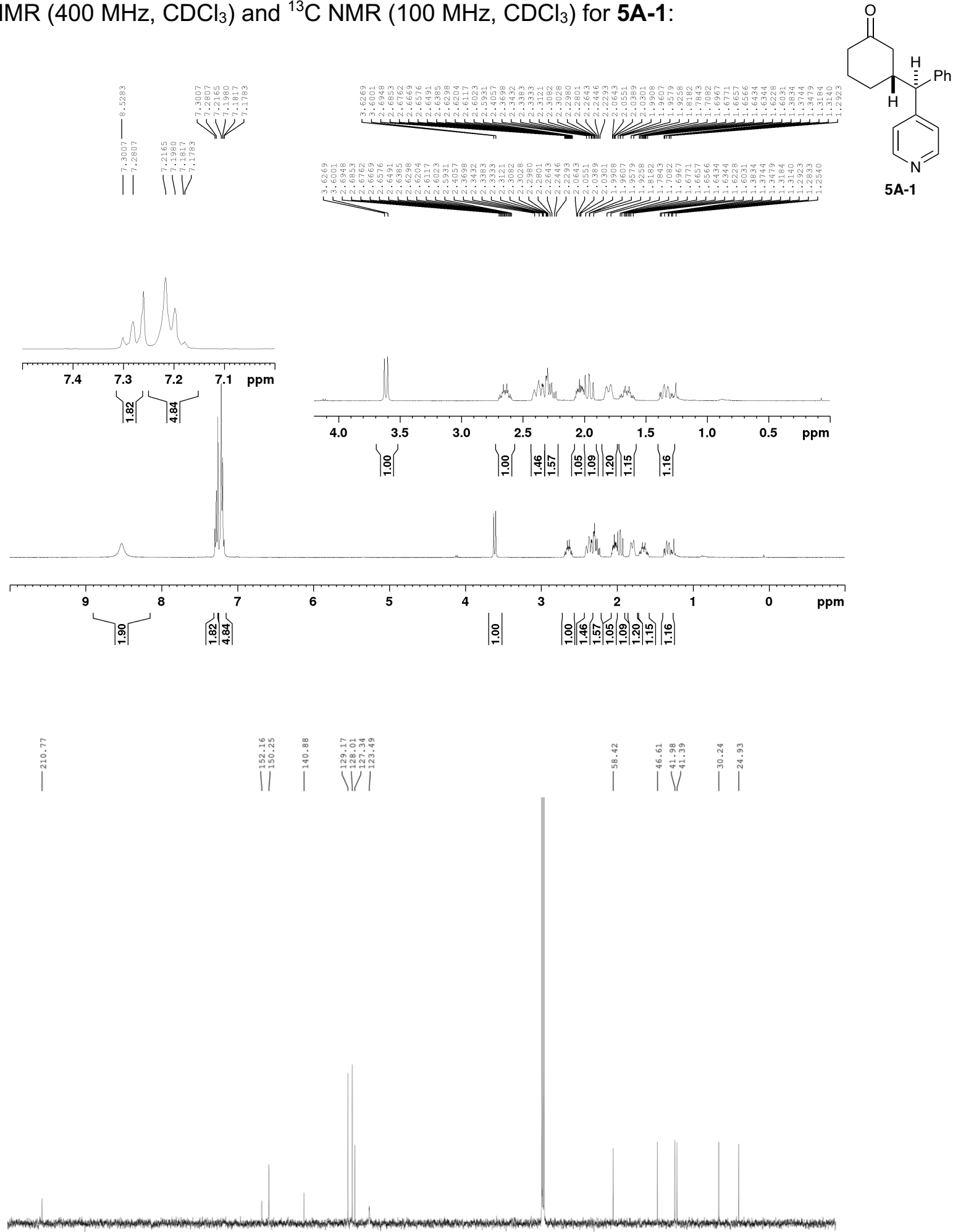

$\begin{array}{llllllllllllllllllll}210 & 200 & 190 & 180 & 170 & 160 & 150 & 140 & 130 & 120 & 110 & 100 & 90 & 80 & 70 & 60 & 50 & 40 & 30 & 20\end{array}$ 
$\cos \left(600 \mathrm{MHz}, \mathrm{C}_{6} \mathrm{H}_{6}\right.$ and $\left.\mathrm{CDCl}_{3}\right)$ for $\mathbf{5 A - 1}$ at $10{ }^{\circ} \mathrm{C}$ :

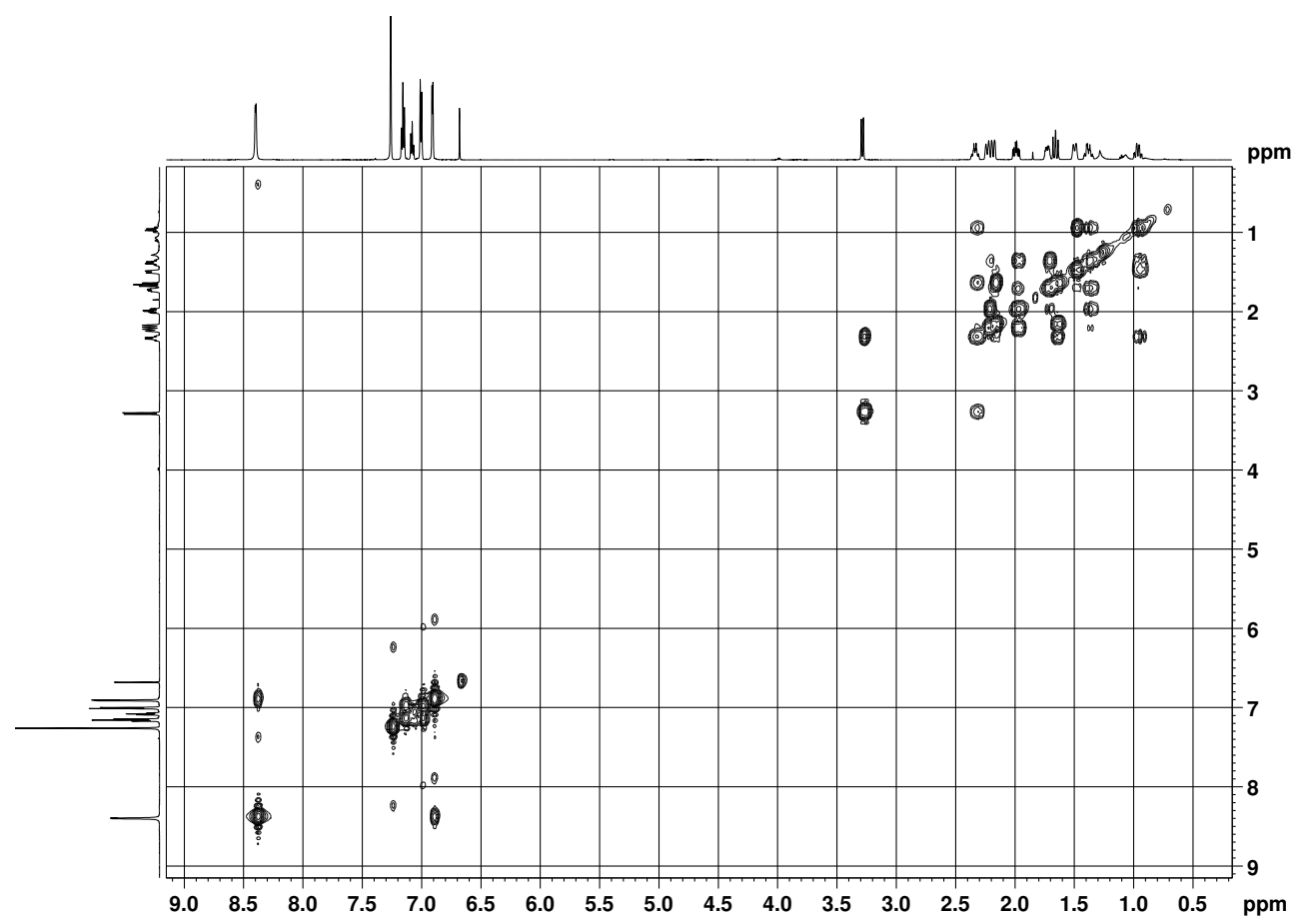

NOESY $\left(600 \mathrm{MHz}, \mathrm{C}_{6} \mathrm{H}_{6}\right.$ and $\left.\mathrm{CDCl}_{3}\right)$ for $5 \mathrm{~A}-1$ recorded at $10{ }^{\circ} \mathrm{C}$ :

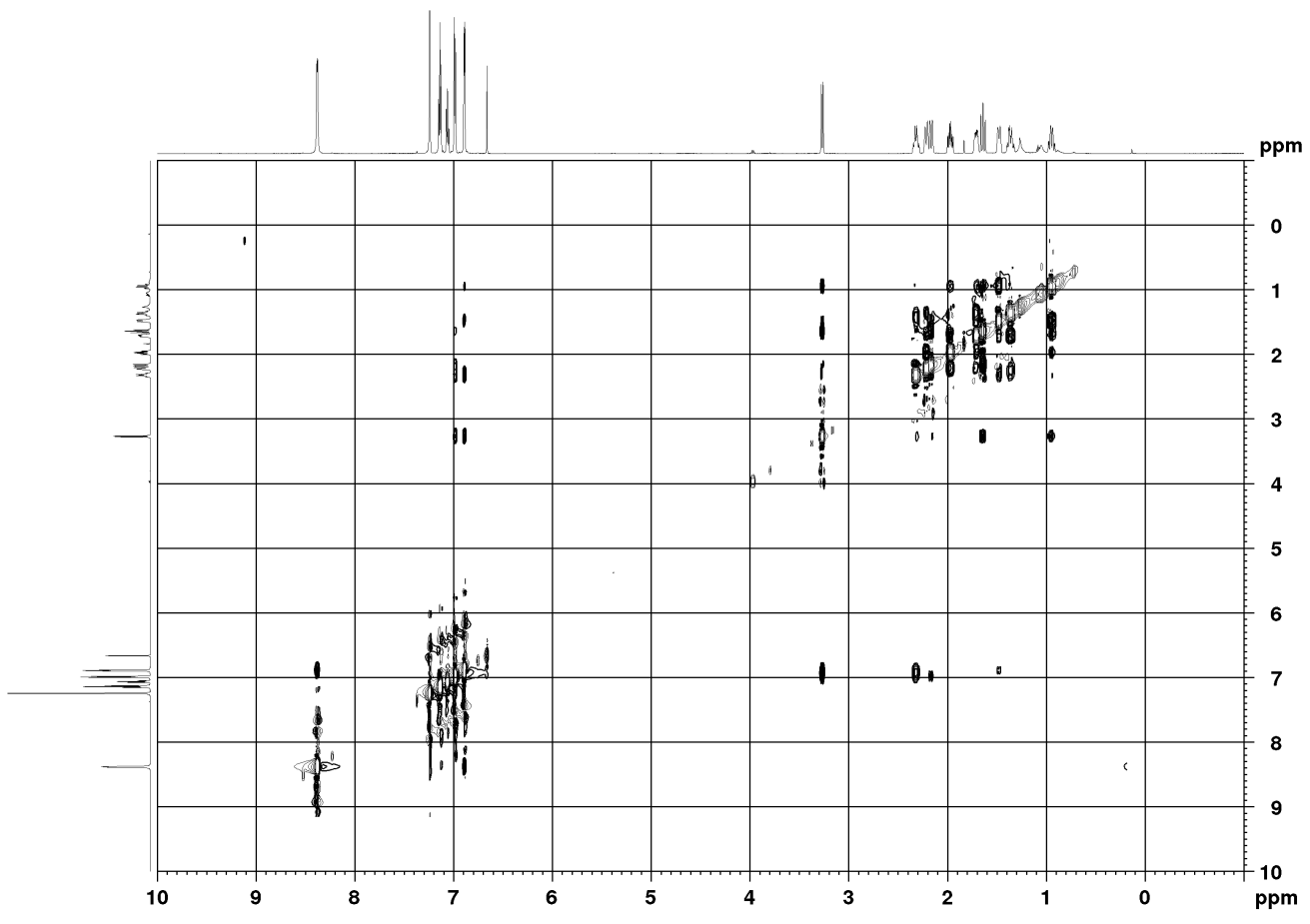


${ }^{1} \mathrm{H} \mathrm{NMR}\left(400 \mathrm{MHz}, \mathrm{CDCl}_{3}\right)$ and ${ }^{13} \mathrm{C} \mathrm{NMR}\left(100 \mathrm{MHz}, \mathrm{CDCl}_{3}\right)$ for 5A-2:
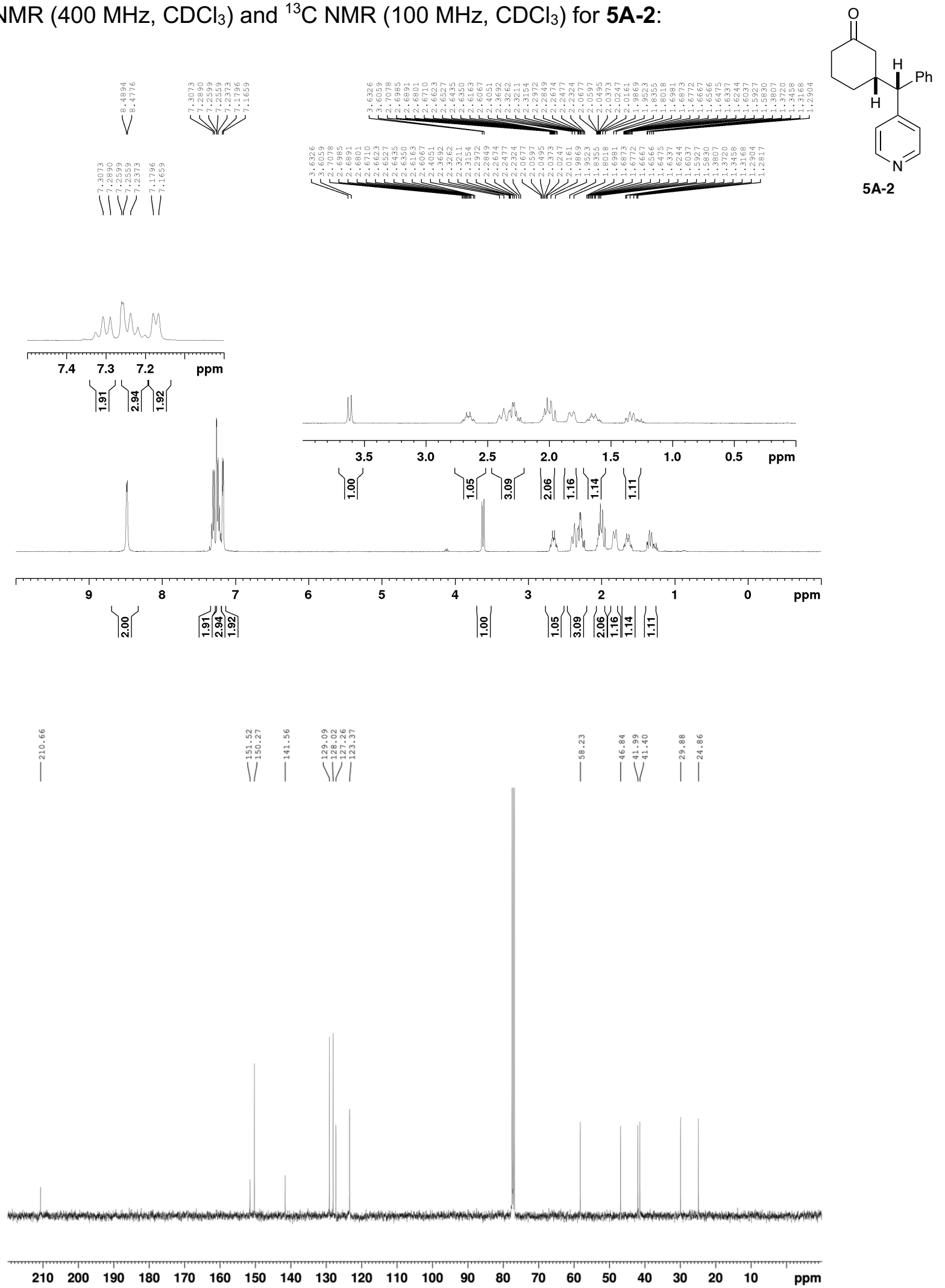
$\cos \left(600 \mathrm{MHz}, \mathrm{C}_{6} \mathrm{H}_{6}\right.$ and $\left.\mathrm{CDCl}_{3}\right)$ for $\mathbf{5 A - 2}$ at $10{ }^{\circ} \mathrm{C}$ :

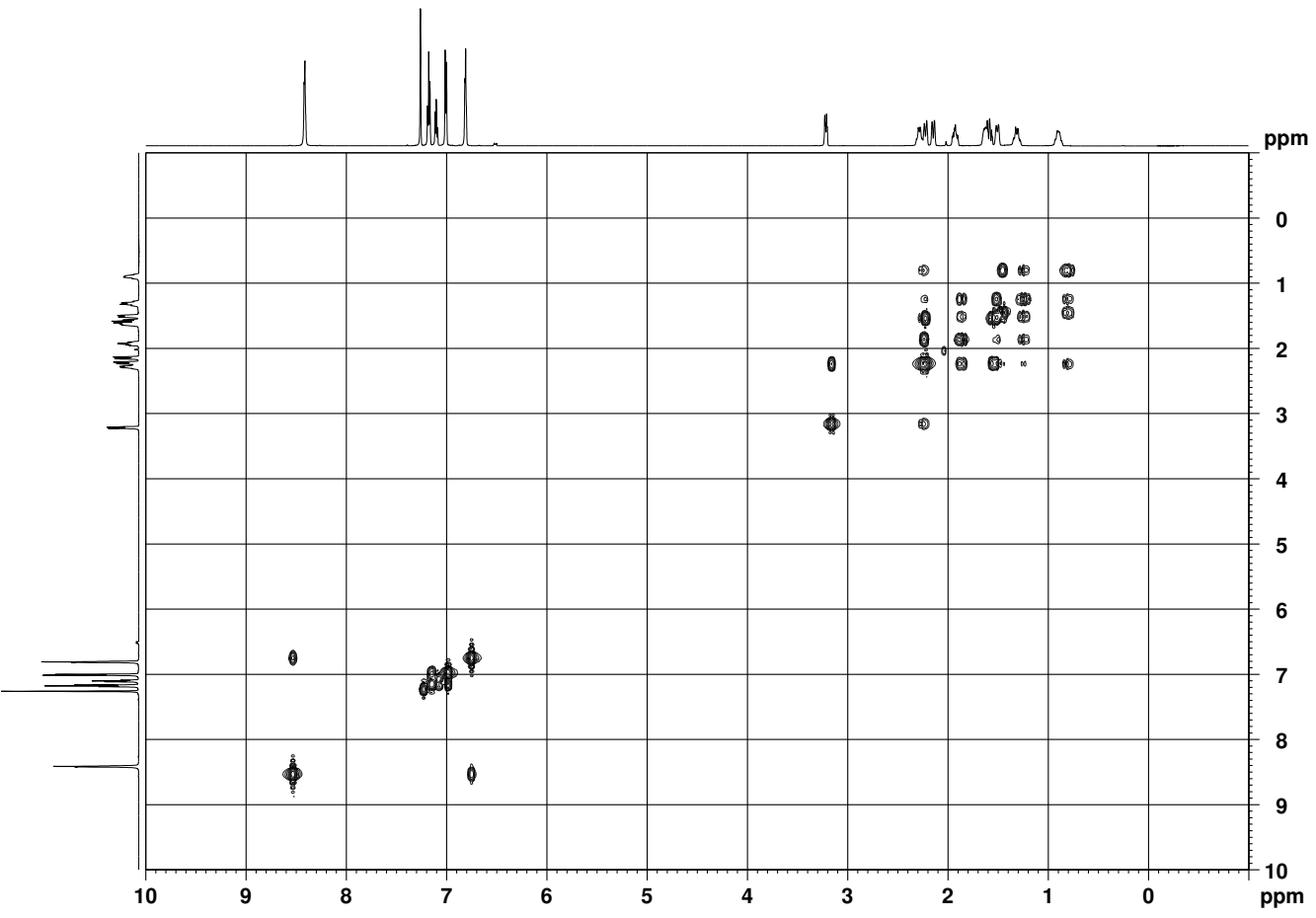

NOESY $\left(600 \mathrm{MHz}, \mathrm{C}_{6} \mathrm{H}_{6}\right.$ and $\left.\mathrm{CDCl}_{3}\right)$ for $\mathbf{5 A - 2}$ at $10{ }^{\circ} \mathrm{C}$ :

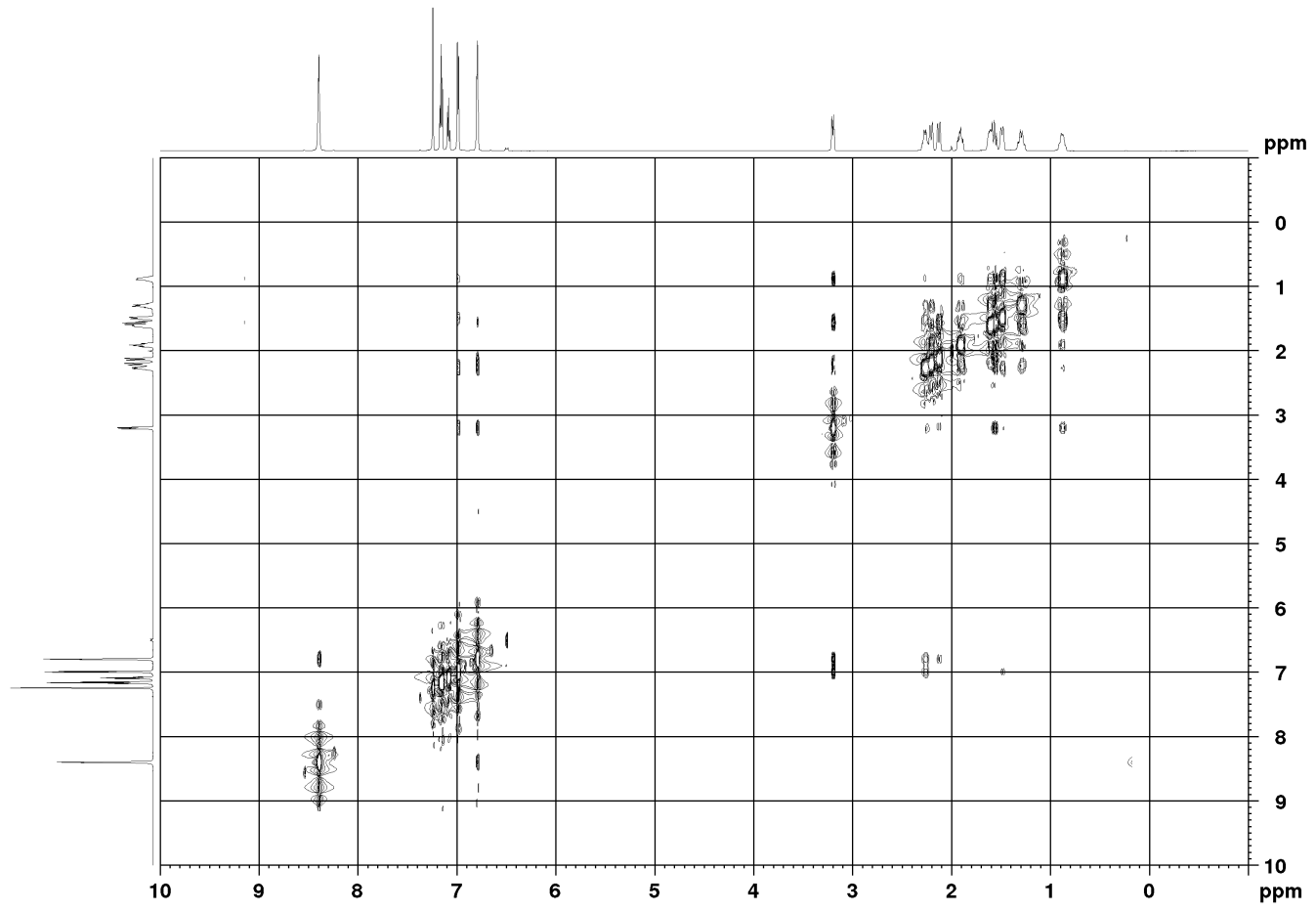


${ }^{1} \mathrm{H}$ NMR $\left(400 \mathrm{MHz}, \mathrm{CDCl}_{3}\right)$ and ${ }^{13} \mathrm{C} \mathrm{NMR}\left(100 \mathrm{MHz}, \mathrm{CDCl}_{3}\right)$ for $6 \mathrm{~A}$ :
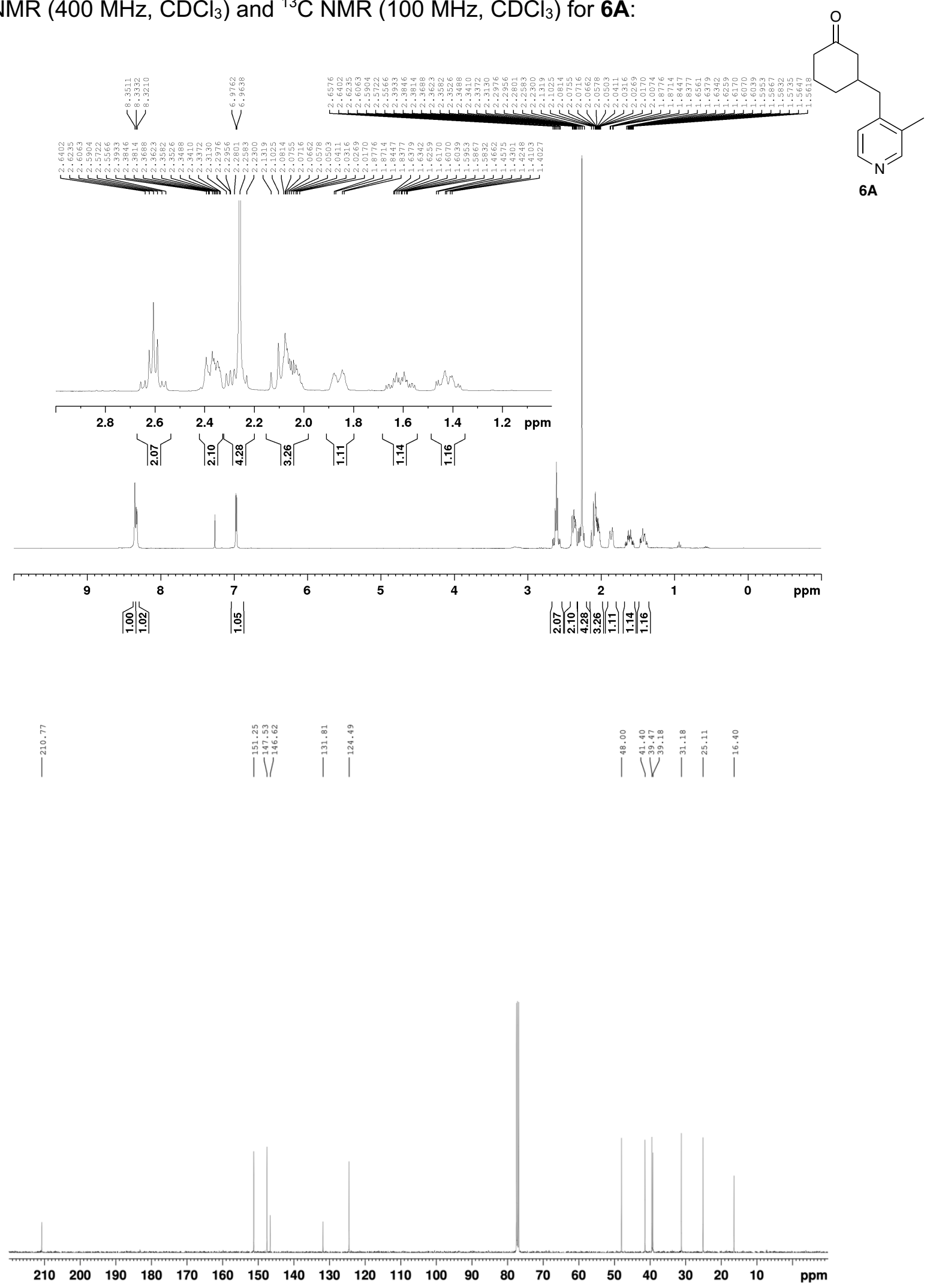
${ }^{1} \mathrm{H} \mathrm{NMR}\left(400 \mathrm{MHz}, \mathrm{CDCl}_{3}\right)$ and ${ }^{13} \mathrm{C} \mathrm{NMR}\left(100 \mathrm{MHz}, \mathrm{CDCl}_{3}\right)$ for 7A:
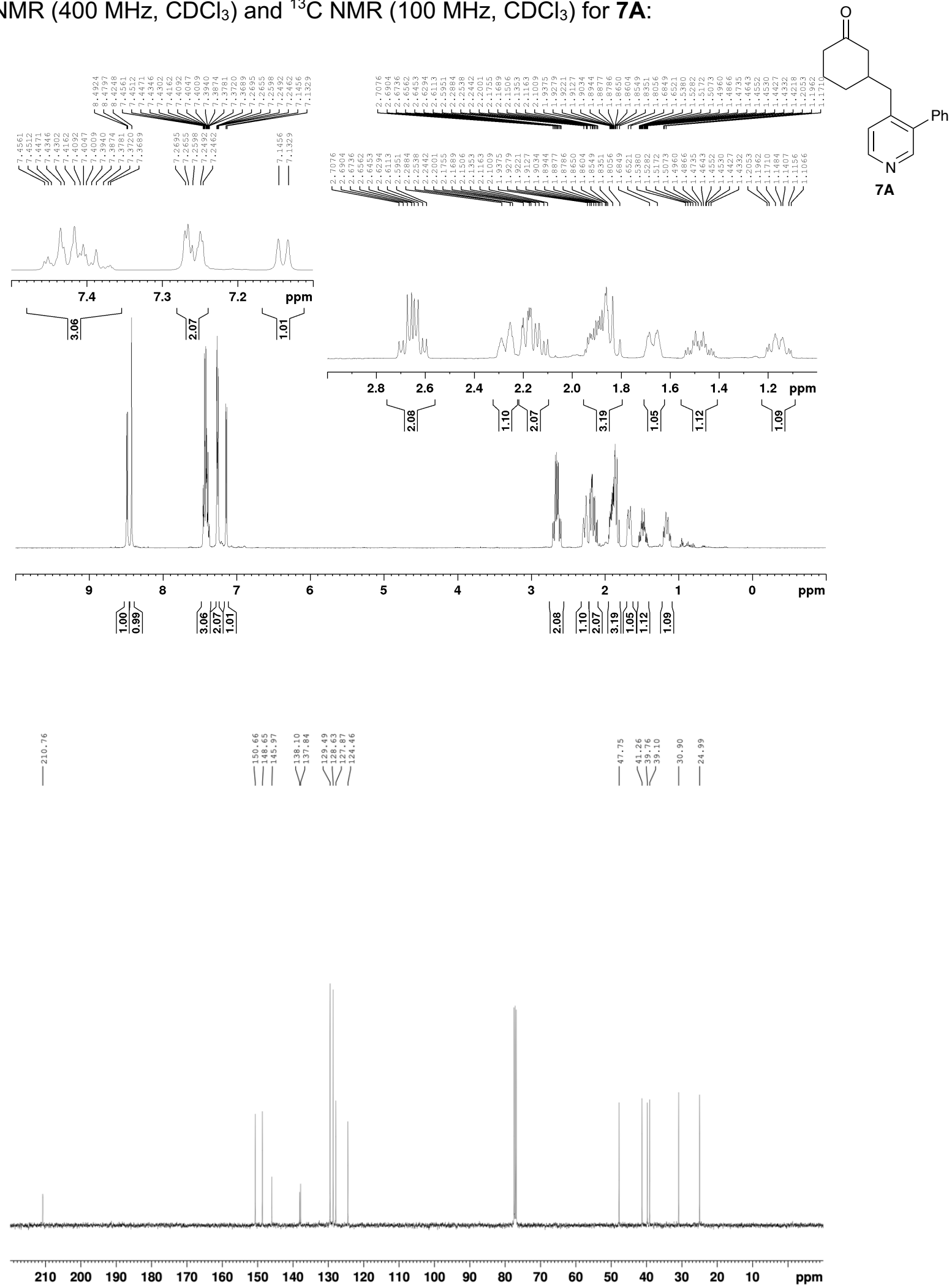
${ }^{1} \mathrm{H}$ NMR (400 MHz, $\left.\mathrm{CDCl}_{3}\right)$ and ${ }^{13} \mathrm{C}$ NMR (100 MHz, $\left.\mathrm{CDCl}_{3}\right)$ for 8A:
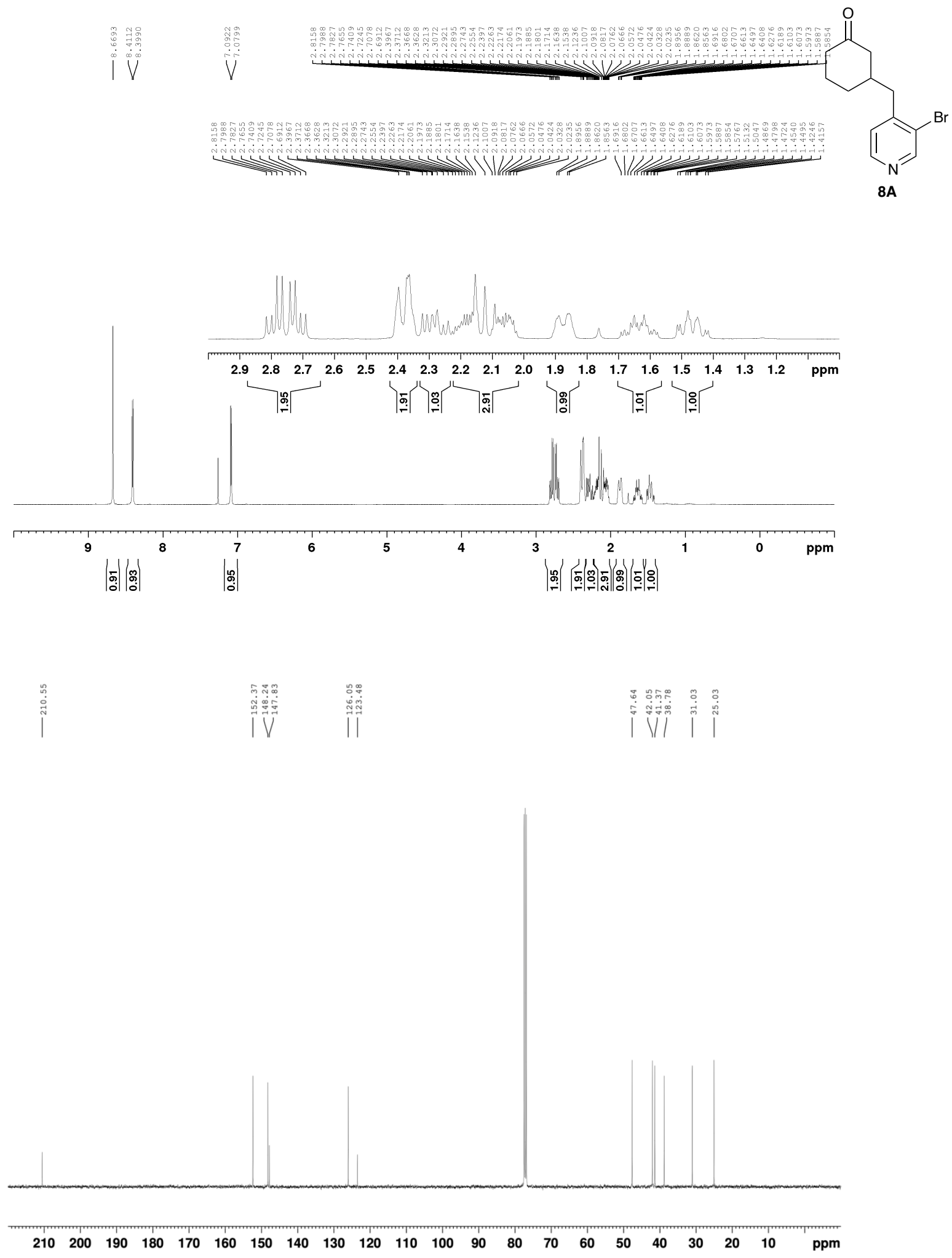
${ }^{1} \mathrm{H}$ NMR (400 MHz, $\left.\mathrm{CDCl}_{3}\right)$ and ${ }^{13} \mathrm{C}$ NMR (100 MHz, $\left.\mathrm{CDCl}_{3}\right)$ for 9A:
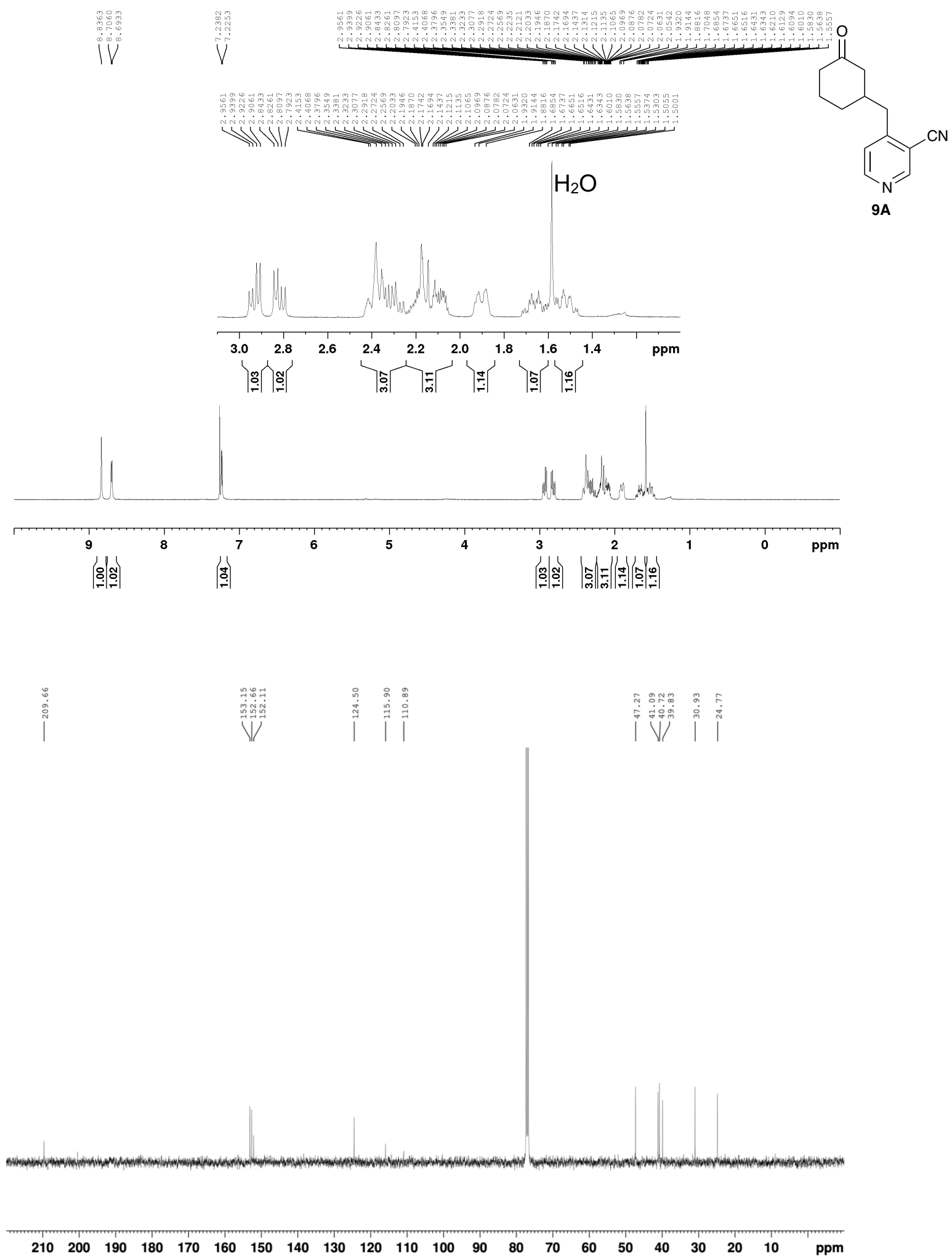
${ }^{1} \mathrm{H}$ NMR $\left(400 \mathrm{MHz}, \mathrm{CDCl}_{3}\right)$ and ${ }^{13} \mathrm{C}$ NMR $\left(100 \mathrm{MHz}, \mathrm{CDCl}_{3}\right)$ for 10A:

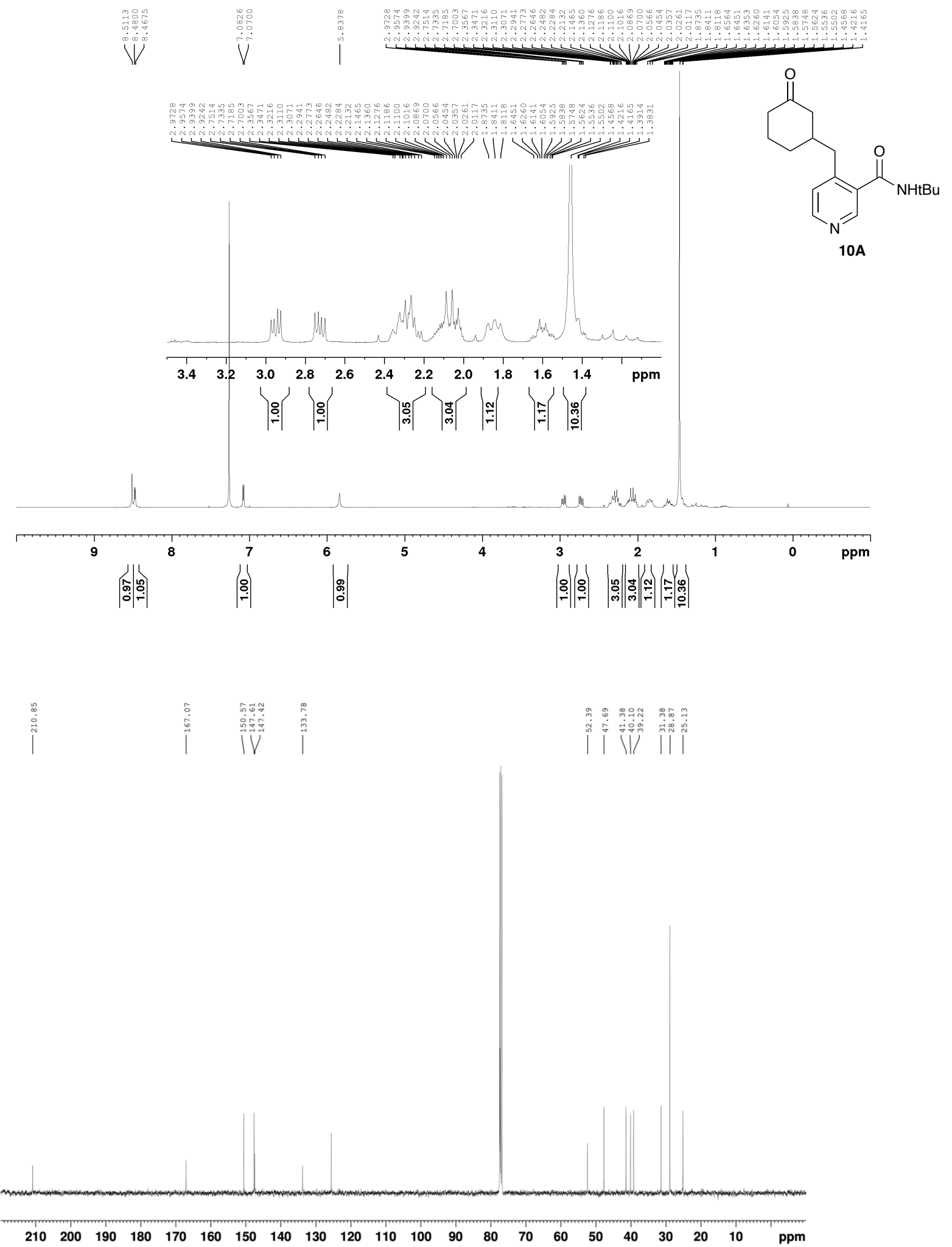


${ }^{1} \mathrm{H} \mathrm{NMR}\left(400 \mathrm{MHz}, \mathrm{CDCl}_{3}\right)$ and ${ }^{13} \mathrm{C} \mathrm{NMR}\left(100 \mathrm{MHz}, \mathrm{CDCl}_{3}\right)$ for 11A:

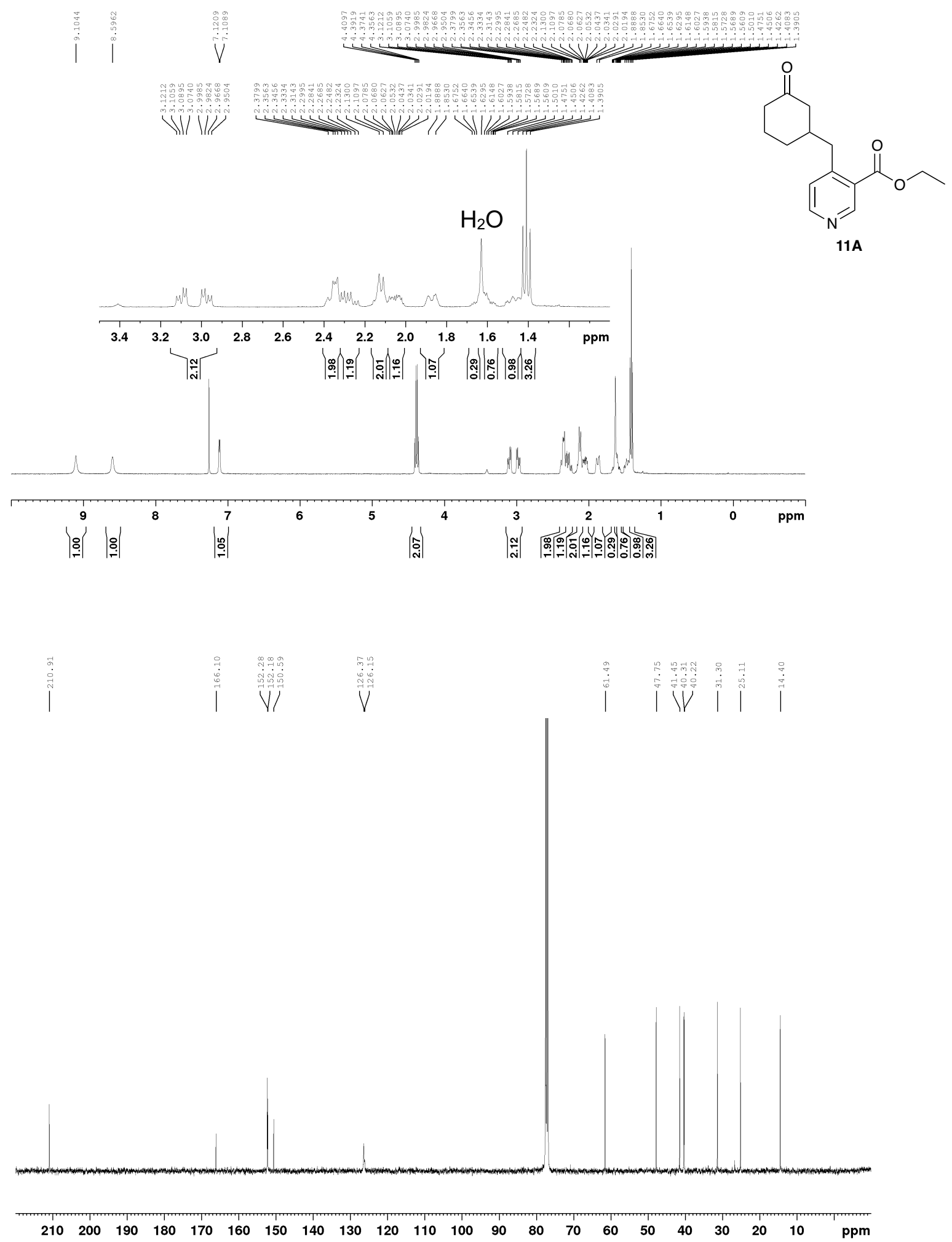


${ }^{1} \mathrm{H} \mathrm{NMR}\left(400 \mathrm{MHz}, \mathrm{CDCl}_{3}\right)$ and ${ }^{13} \mathrm{C} \mathrm{NMR}\left(100 \mathrm{MHz}, \mathrm{CDCl}_{3}\right)$ for 12A:

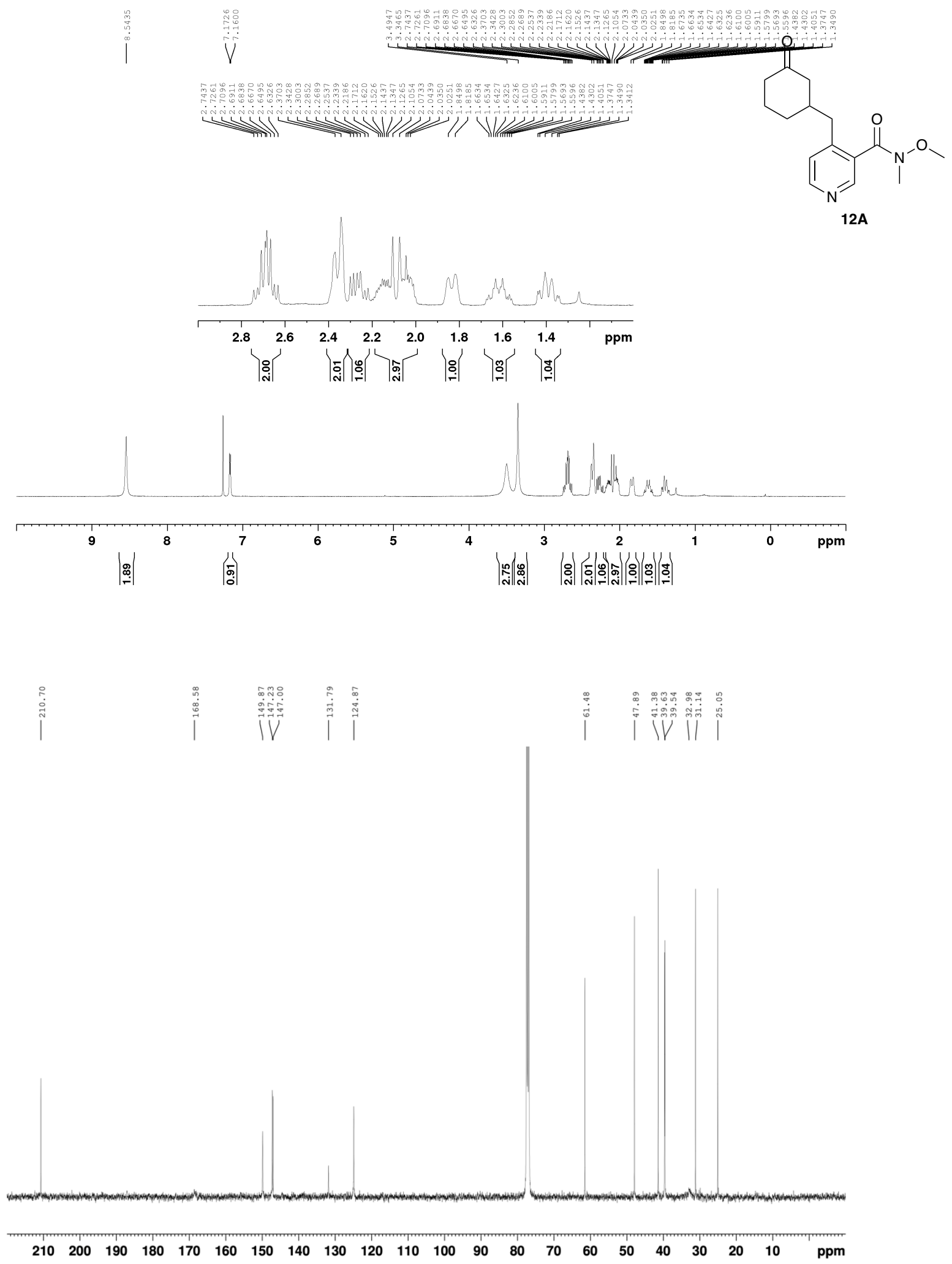


${ }^{1} \mathrm{H} \mathrm{NMR}\left(400 \mathrm{MHz}, \mathrm{CDCl}_{3}\right)$ and ${ }^{13} \mathrm{C} \mathrm{NMR}\left(100 \mathrm{MHz}, \mathrm{CDCl}_{3}\right)$ for 13A:

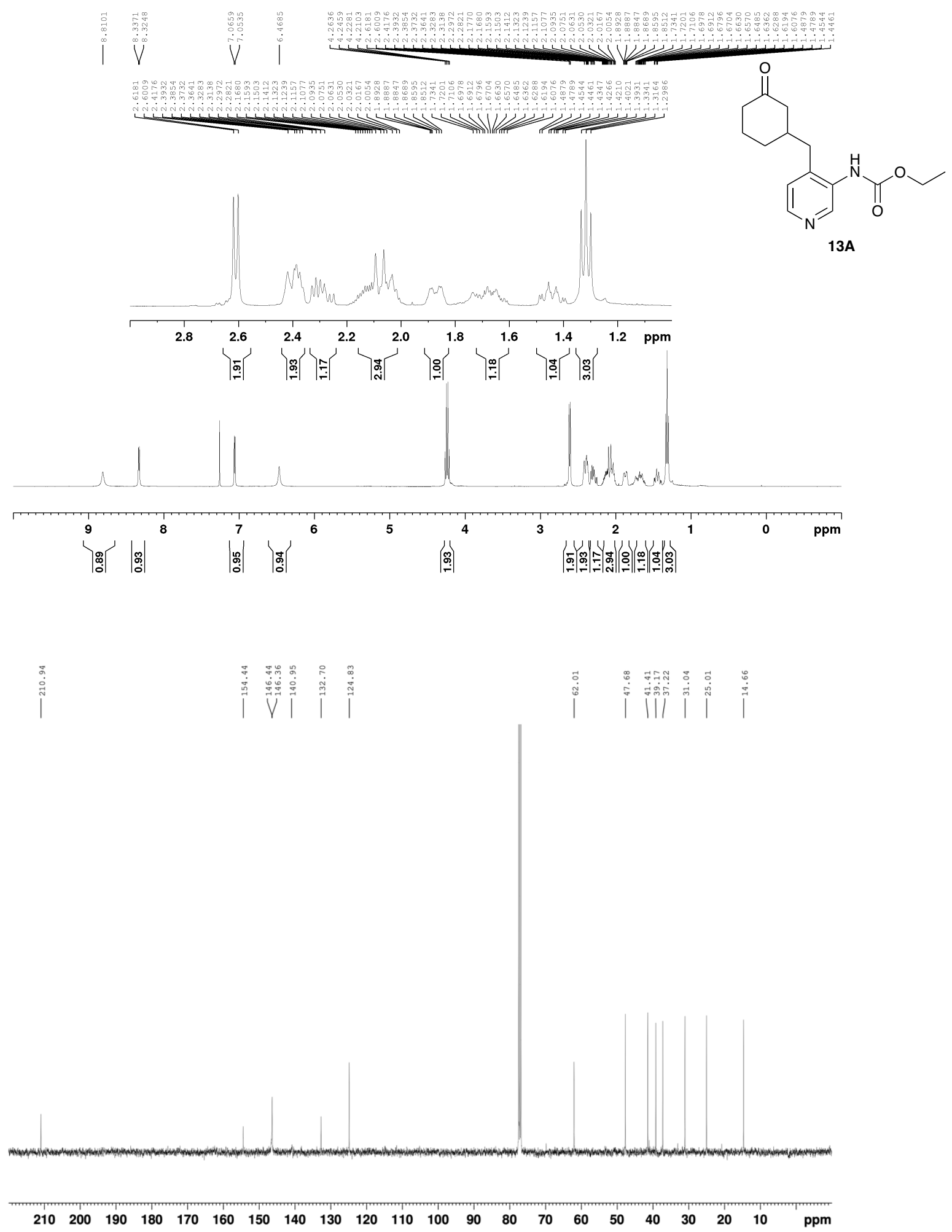


${ }^{1} \mathrm{H}$ NMR $\left(400 \mathrm{MHz}, \mathrm{CDCl}_{3}\right)$ and ${ }^{13} \mathrm{C}$ NMR $\left(100 \mathrm{MHz}, \mathrm{CDCl}_{3}\right)$ for 14A:

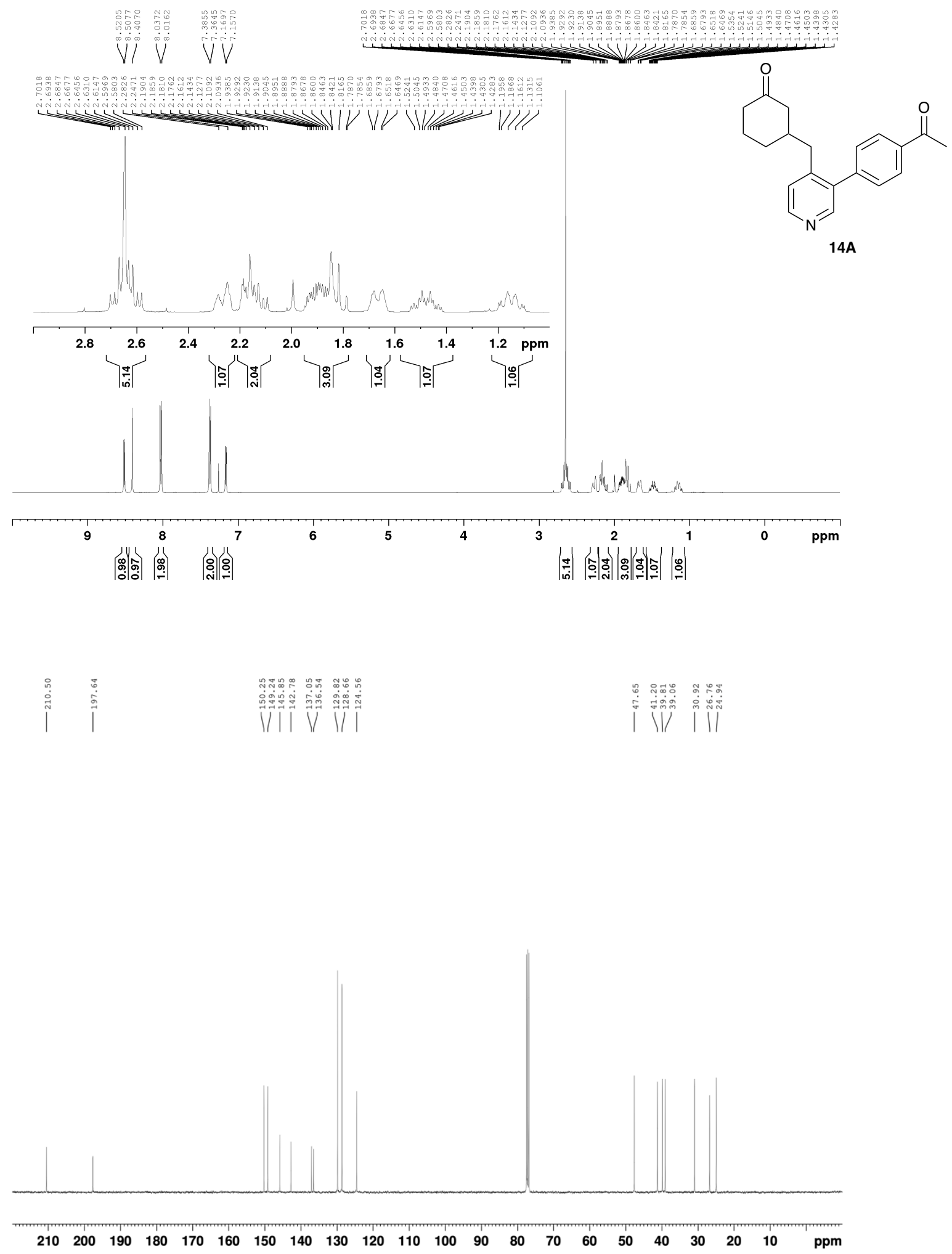


${ }^{1} \mathrm{H}$ NMR $\left(400 \mathrm{MHz}, \mathrm{CDCl}_{3}\right)$ and ${ }^{13} \mathrm{C}$ NMR $\left(100 \mathrm{MHz}, \mathrm{CDCl}_{3}\right)$ for 15A:
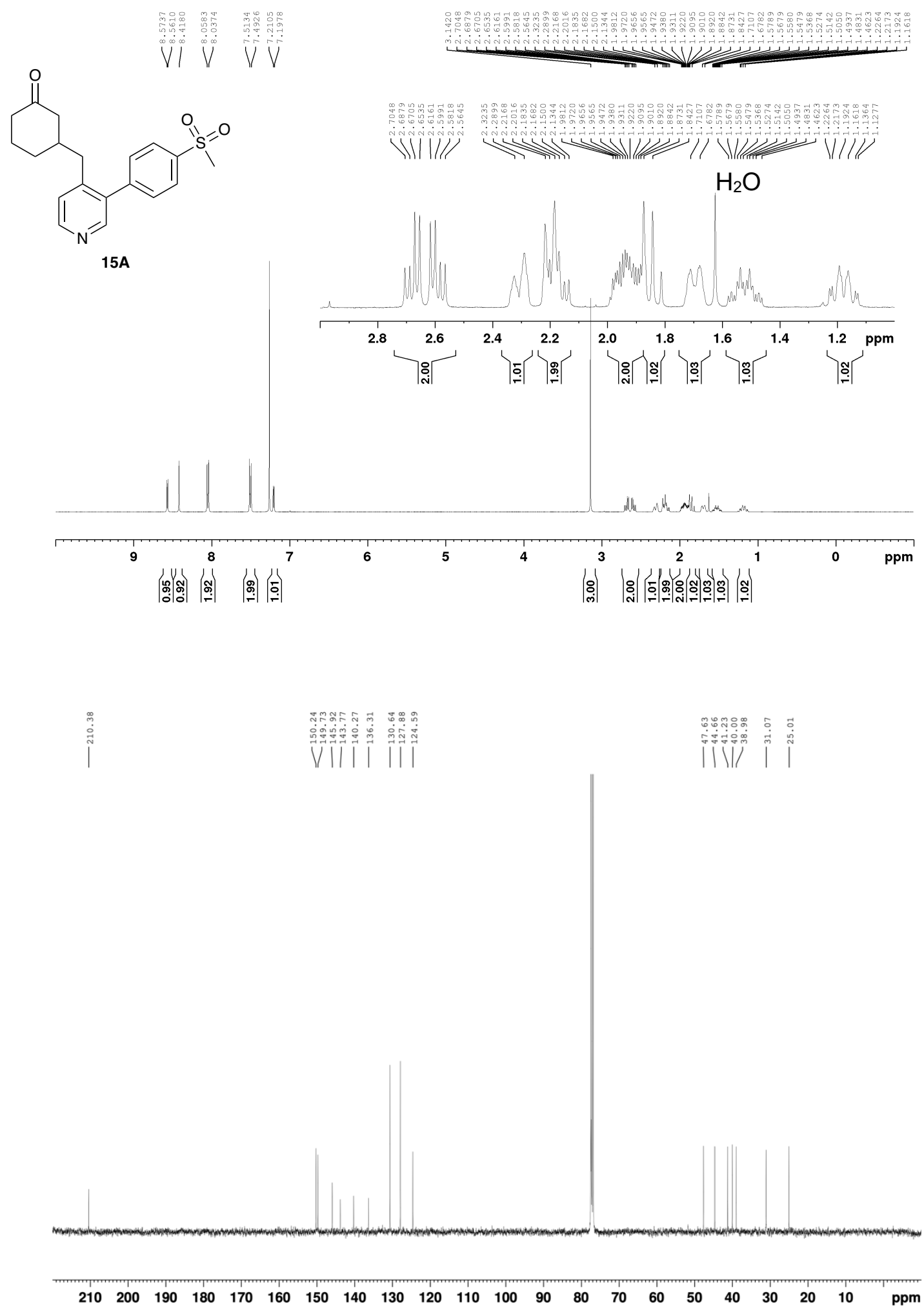
${ }^{1} \mathrm{H} \mathrm{NMR}\left(400 \mathrm{MHz}, \mathrm{CDCl}_{3}\right)$ and ${ }^{13} \mathrm{C} \mathrm{NMR}\left(100 \mathrm{MHz}, \mathrm{CDCl}_{3}\right)$ for 16A:

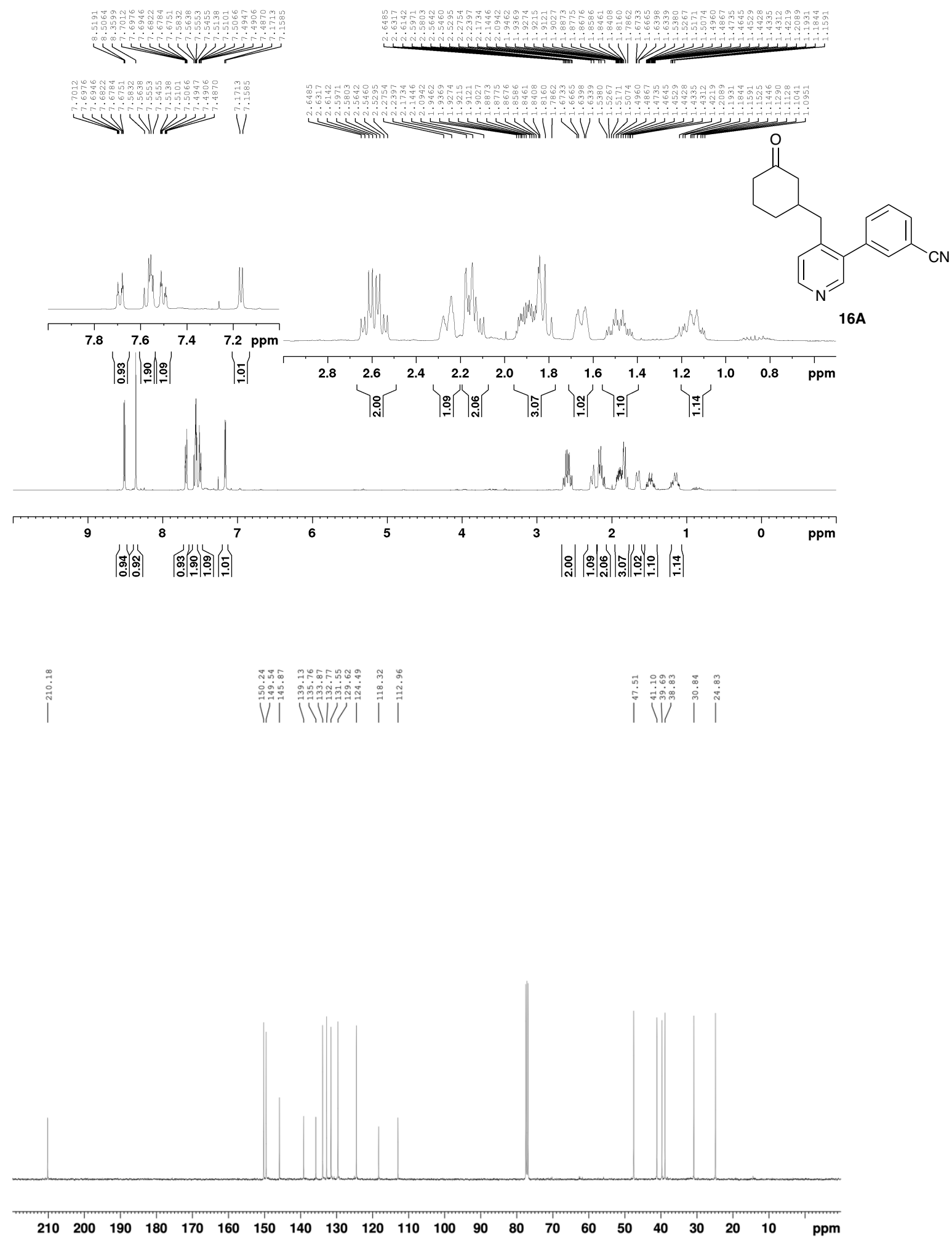


${ }^{1} \mathrm{H}$ NMR $\left(400 \mathrm{MHz}, \mathrm{CDCl}_{3}\right)$ and ${ }^{13} \mathrm{C} \mathrm{NMR}\left(100 \mathrm{MHz}, \mathrm{CDCl}_{3}\right)$ for 17A:

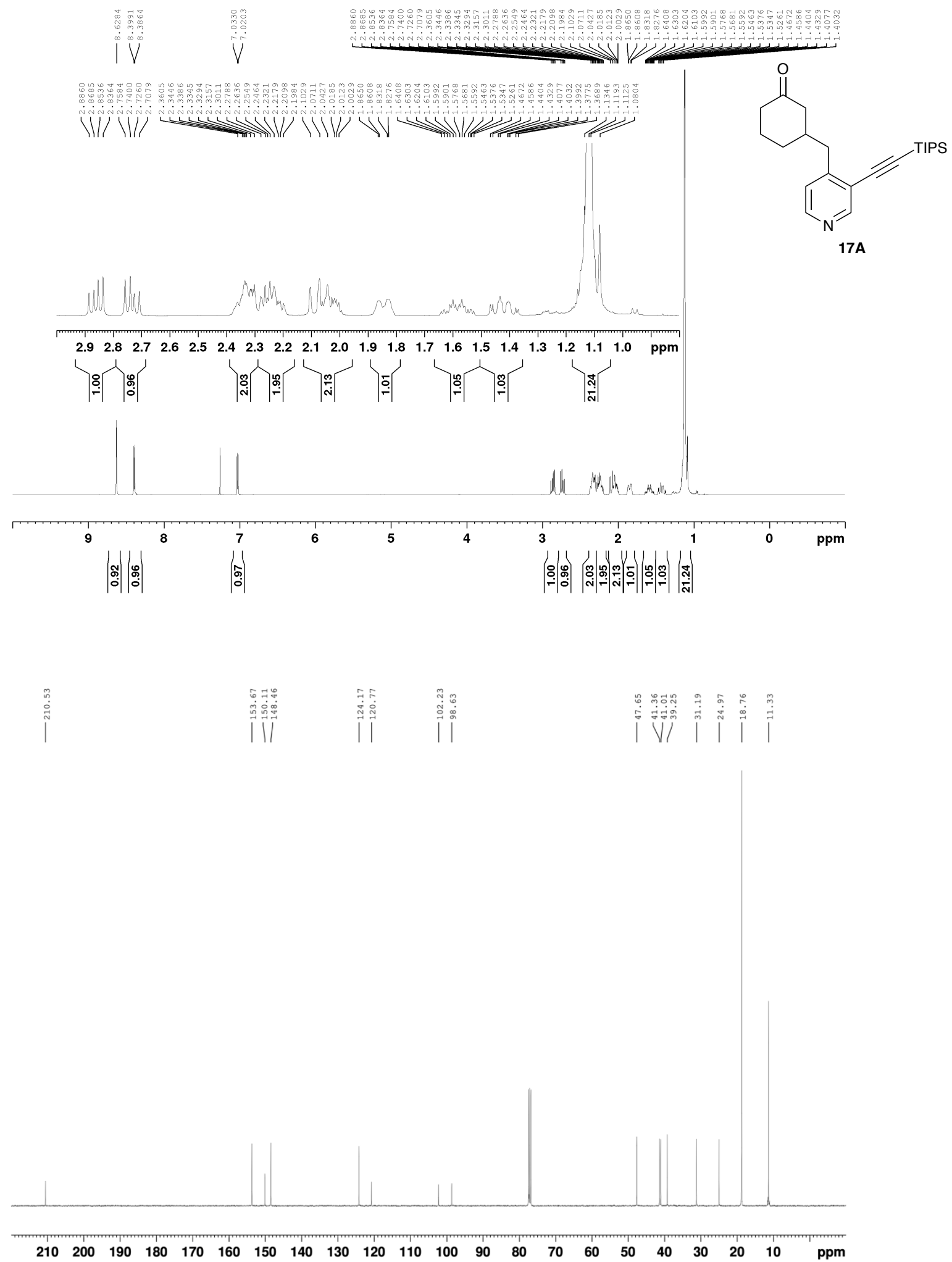


${ }^{1} \mathrm{H}$ NMR $\left(400 \mathrm{MHz}, \mathrm{CDCl}_{3}\right)$ and ${ }^{13} \mathrm{C} \mathrm{NMR}\left(100 \mathrm{MHz}, \mathrm{CDCl}_{3}\right)$ for 18A:

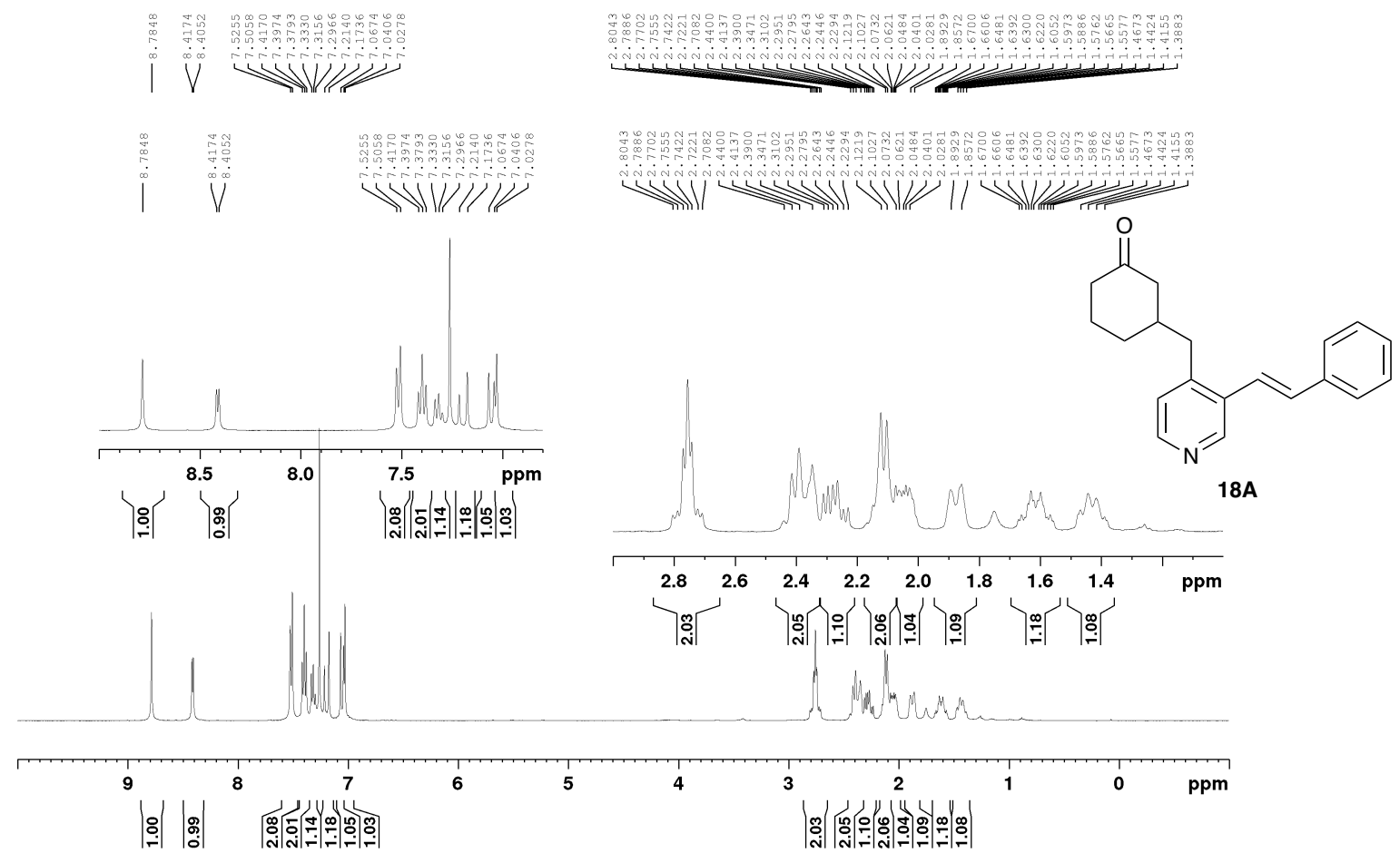

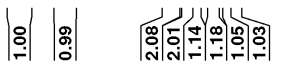

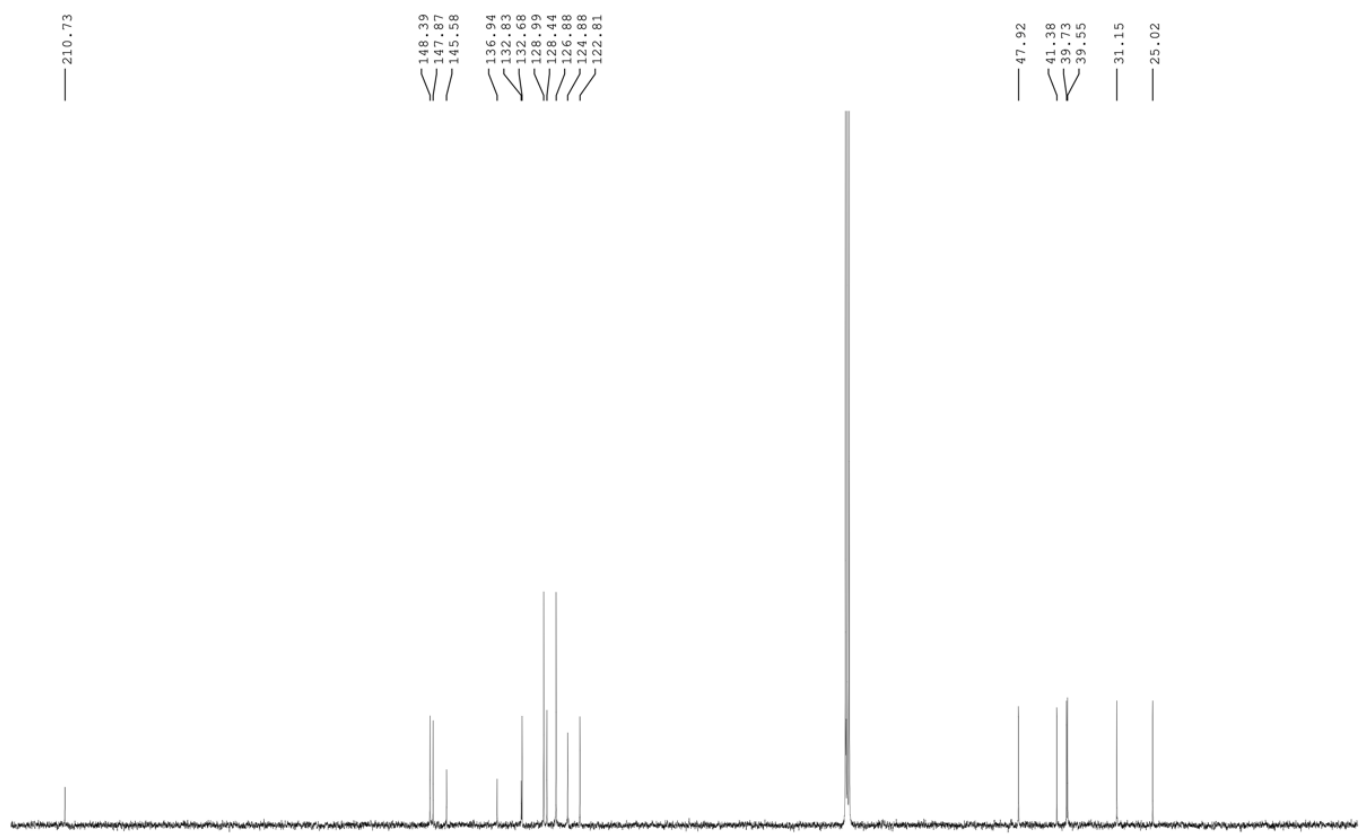

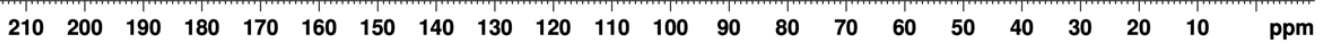


${ }^{1} \mathrm{H}$ NMR (400 MHz, $\left.\mathrm{CDCl}_{3}\right)$ and ${ }^{13} \mathrm{C}$ NMR (100 MHz, $\left.\mathrm{CDCl}_{3}\right)$ for 2B:

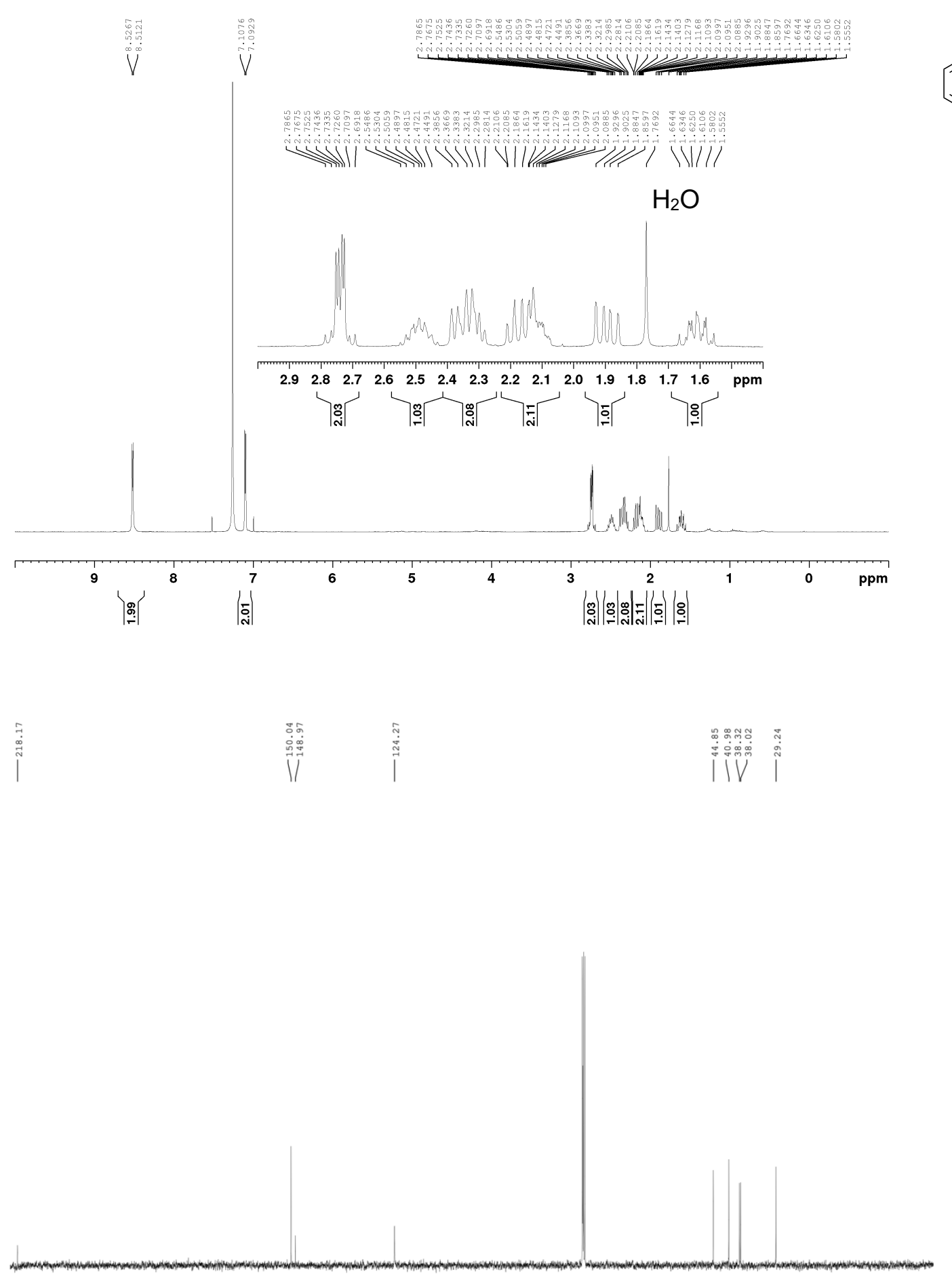


${ }^{1} \mathrm{H} \mathrm{NMR}\left(400 \mathrm{MHz}, \mathrm{CDCl}_{3}\right)$ and ${ }^{13} \mathrm{C} \mathrm{NMR}\left(100 \mathrm{MHz}, \mathrm{CDCl}_{3}\right)$ for $\mathbf{2 C}$ :
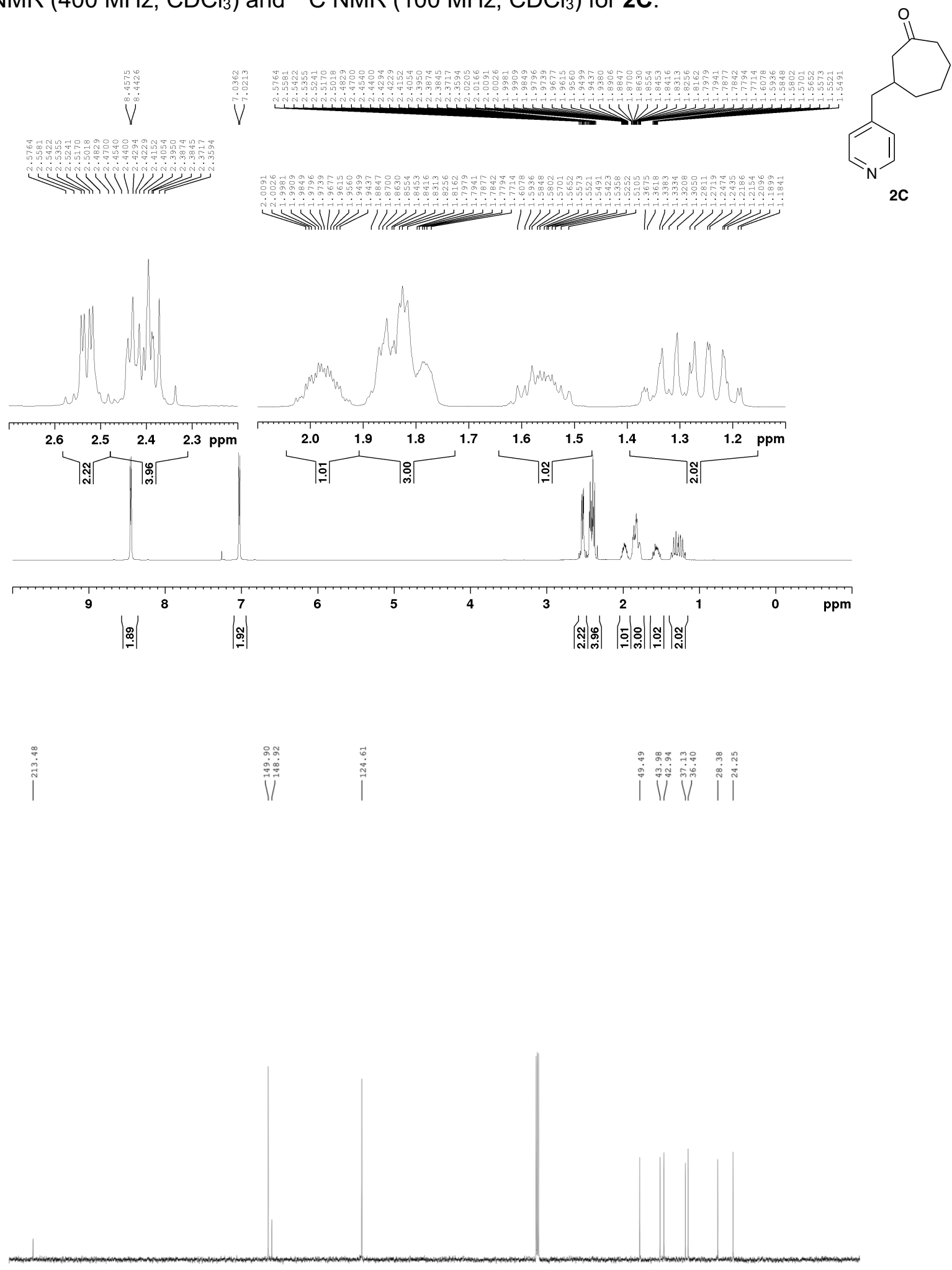

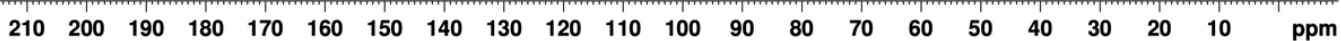


${ }^{1} \mathrm{H}$ NMR $\left(400 \mathrm{MHz}, \mathrm{CDCl}_{3}\right)$ and ${ }^{13} \mathrm{C} \mathrm{NMR}\left(100 \mathrm{MHz}, \mathrm{CDCl}_{3}\right)$ for $\mathbf{2 C}$ ':
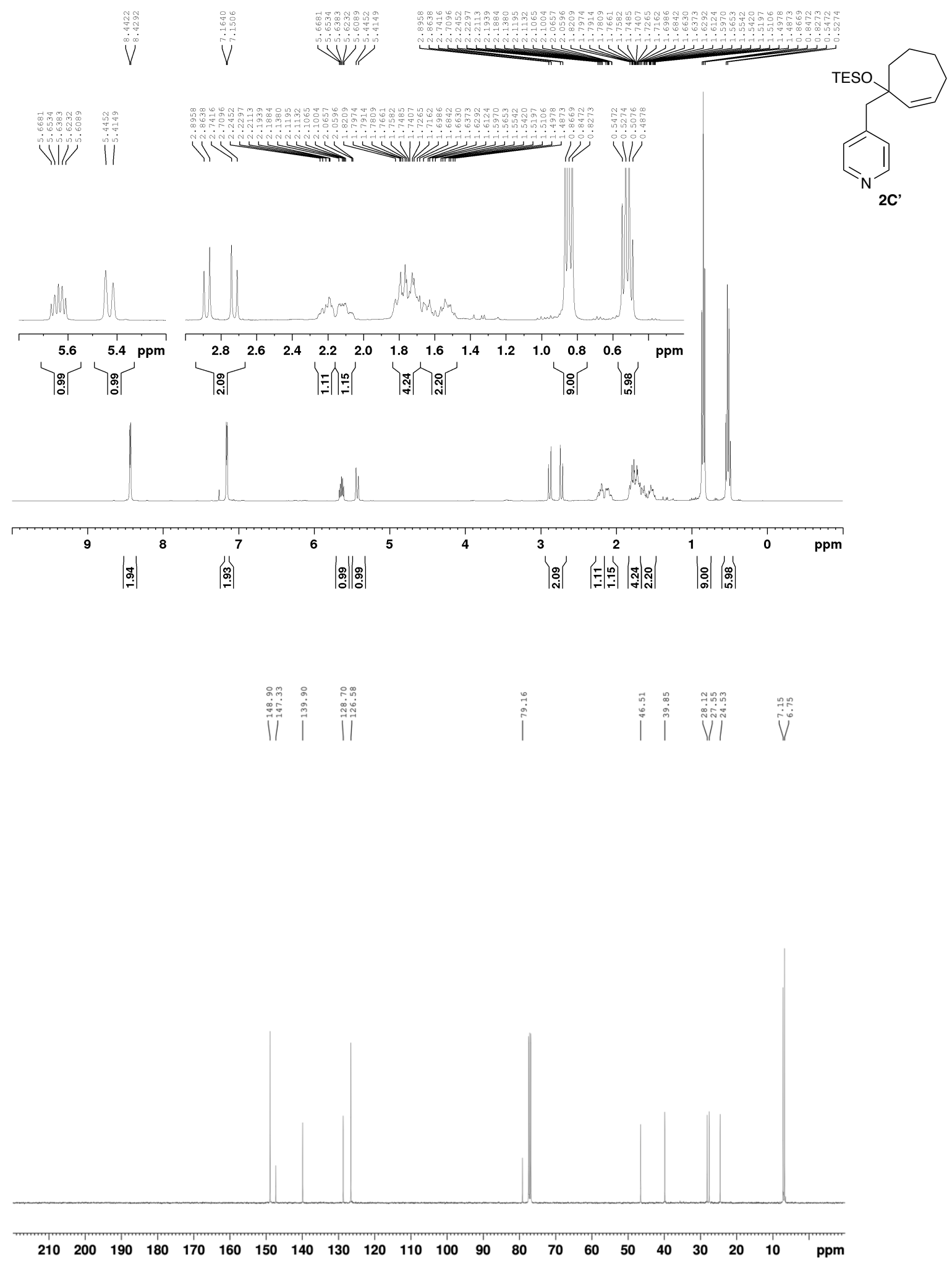
${ }^{1} \mathrm{H} \mathrm{NMR}\left(400 \mathrm{MHz}, \mathrm{CDCl}_{3}\right)$ and $\left.{ }^{13} \mathrm{C} \mathrm{NMR} \mathrm{(100} \mathrm{MHz,} \mathrm{CDCl}_{3}\right)$ for 2D:
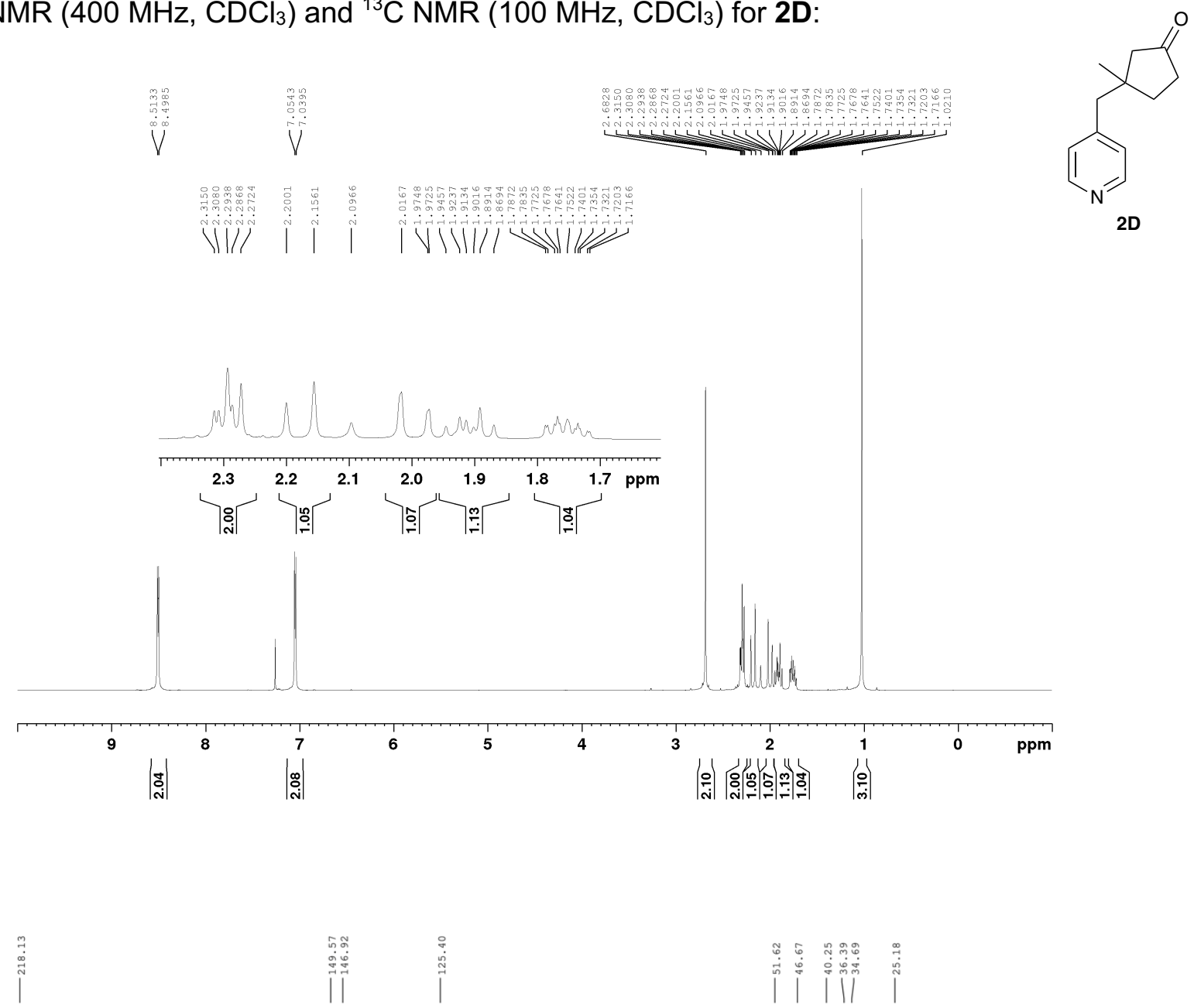

2D

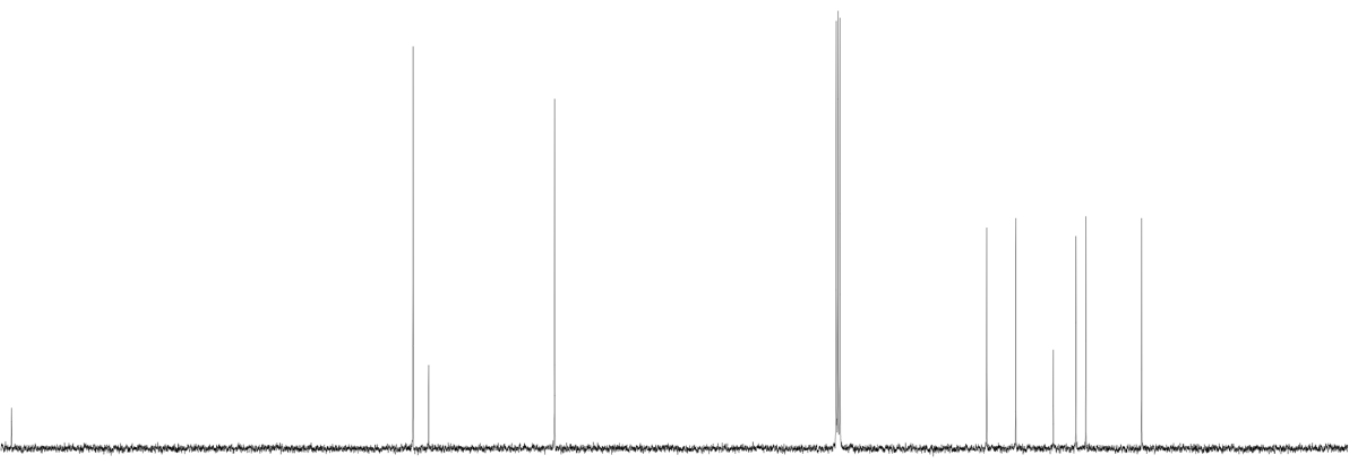

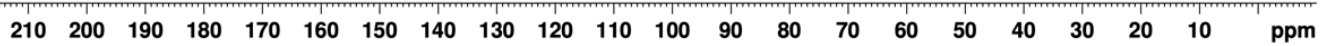


${ }^{1} \mathrm{H} \mathrm{NMR}\left(400 \mathrm{MHz}, \mathrm{CDCl}_{3}\right)$ and ${ }^{13} \mathrm{C} \mathrm{NMR}\left(100 \mathrm{MHz}, \mathrm{CDCl}_{3}\right)$ for 2D':
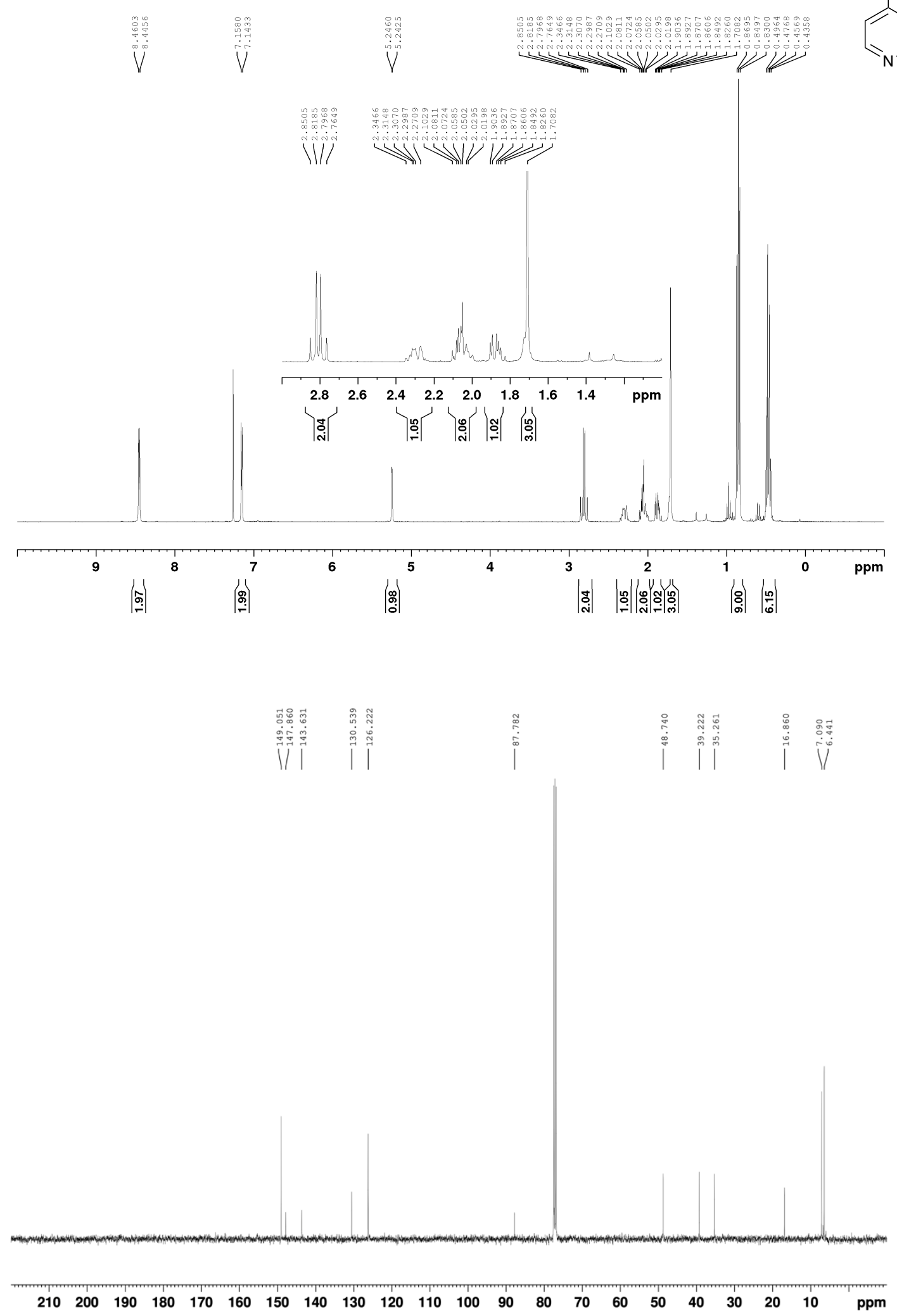
${ }^{1} \mathrm{H} \mathrm{NMR}\left(400 \mathrm{MHz}, \mathrm{CDCl}_{3}\right)$ and ${ }^{13} \mathrm{C} \mathrm{NMR}\left(100 \mathrm{MHz}, \mathrm{CDCl}_{3}\right)$ for 2E:
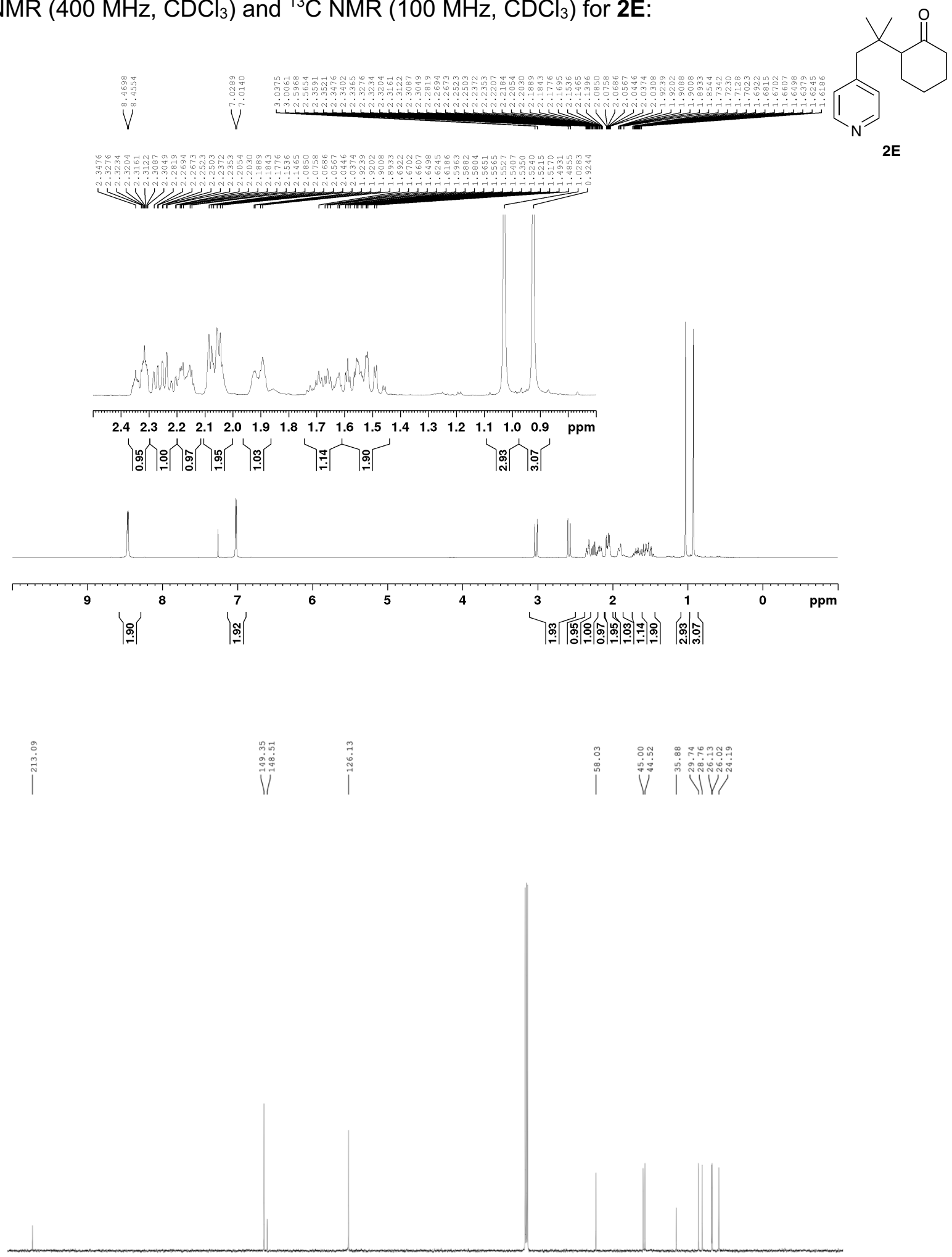

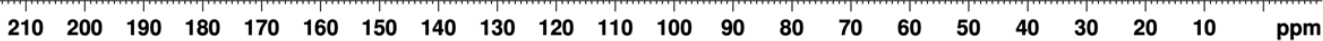


${ }^{1} \mathrm{H} \mathrm{NMR}\left(400 \mathrm{MHz}, \mathrm{CDCl}_{3}\right)$ and ${ }^{13} \mathrm{C} \mathrm{NMR}\left(100 \mathrm{MHz}, \mathrm{CDCl}_{3}\right)$ for $\mathbf{2 F}$ :
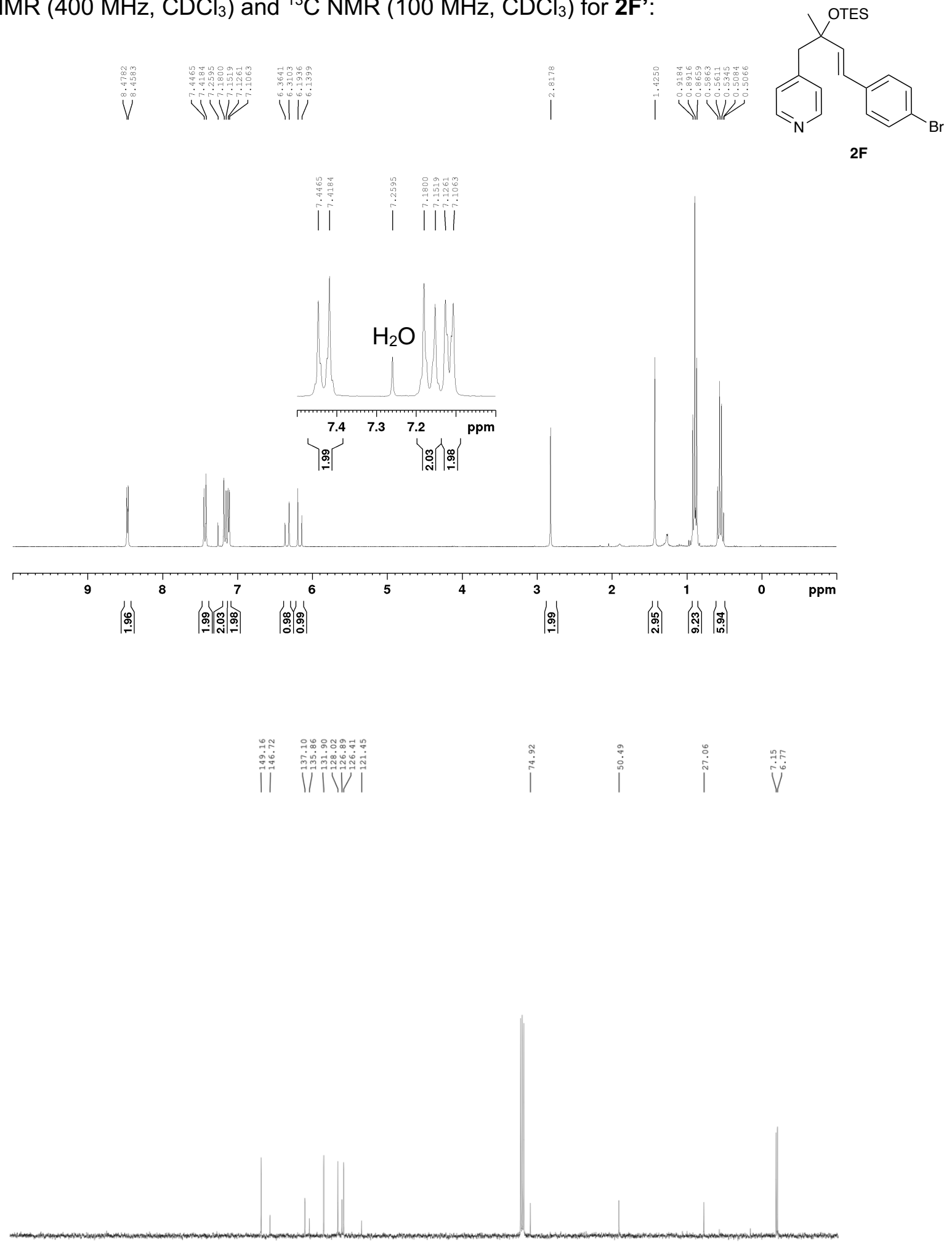

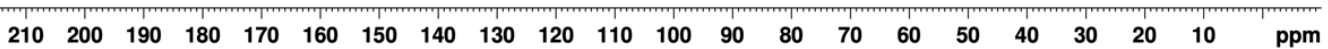


${ }^{1} \mathrm{H}$ NMR $\left(400 \mathrm{MHz}, \mathrm{CDCl}_{3}\right)$ and ${ }^{13} \mathrm{C} \mathrm{NMR}\left(100 \mathrm{MHz}, \mathrm{CDCl}_{3}\right)$ for 2G:
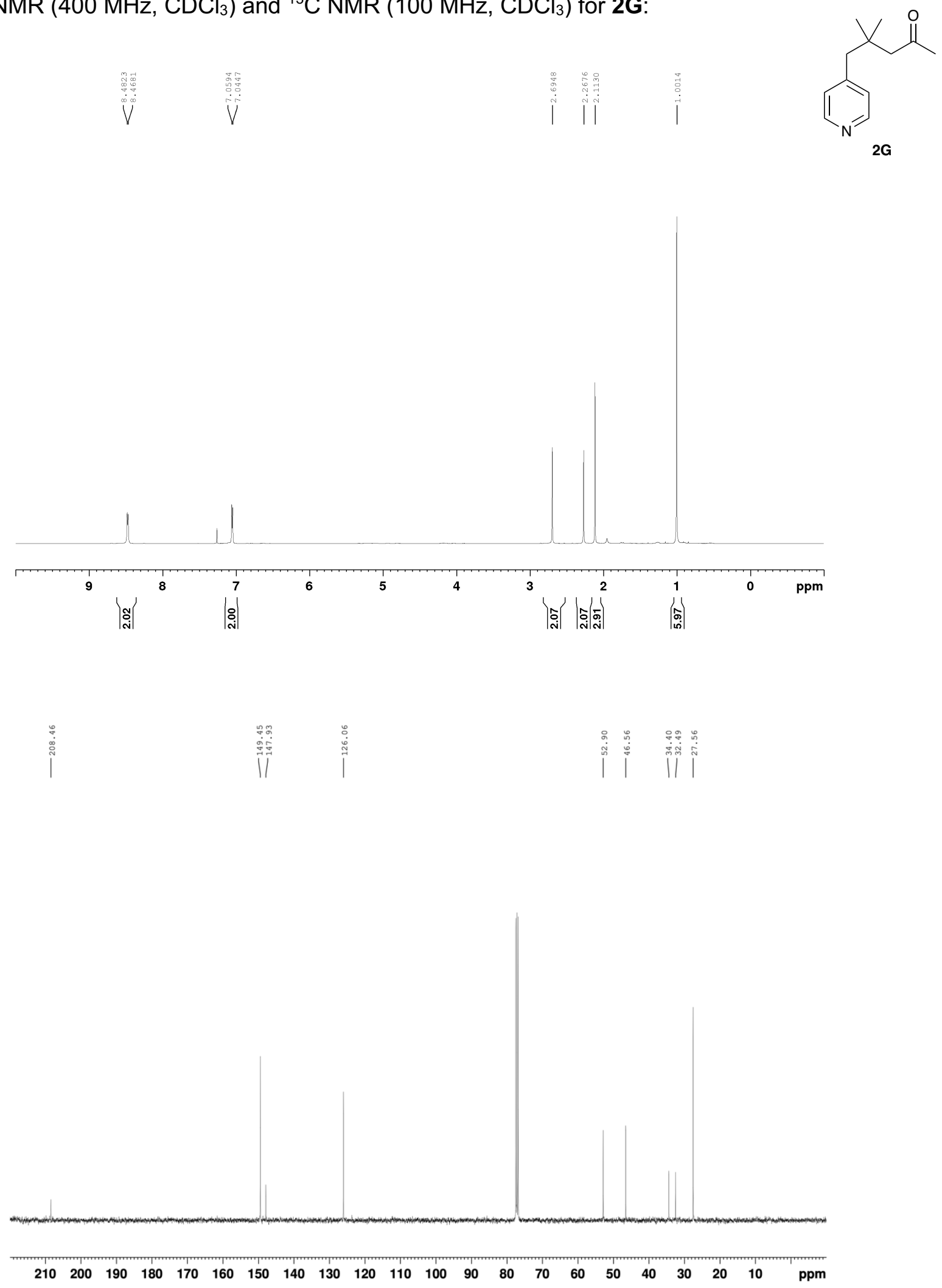
${ }^{1} \mathrm{H} \mathrm{NMR}\left(400 \mathrm{MHz}, \mathrm{CDCl}_{3}\right)$ and ${ }^{13} \mathrm{C} \mathrm{NMR}\left(100 \mathrm{MHz}, \mathrm{CDCl}_{3}\right)$ for 2G':
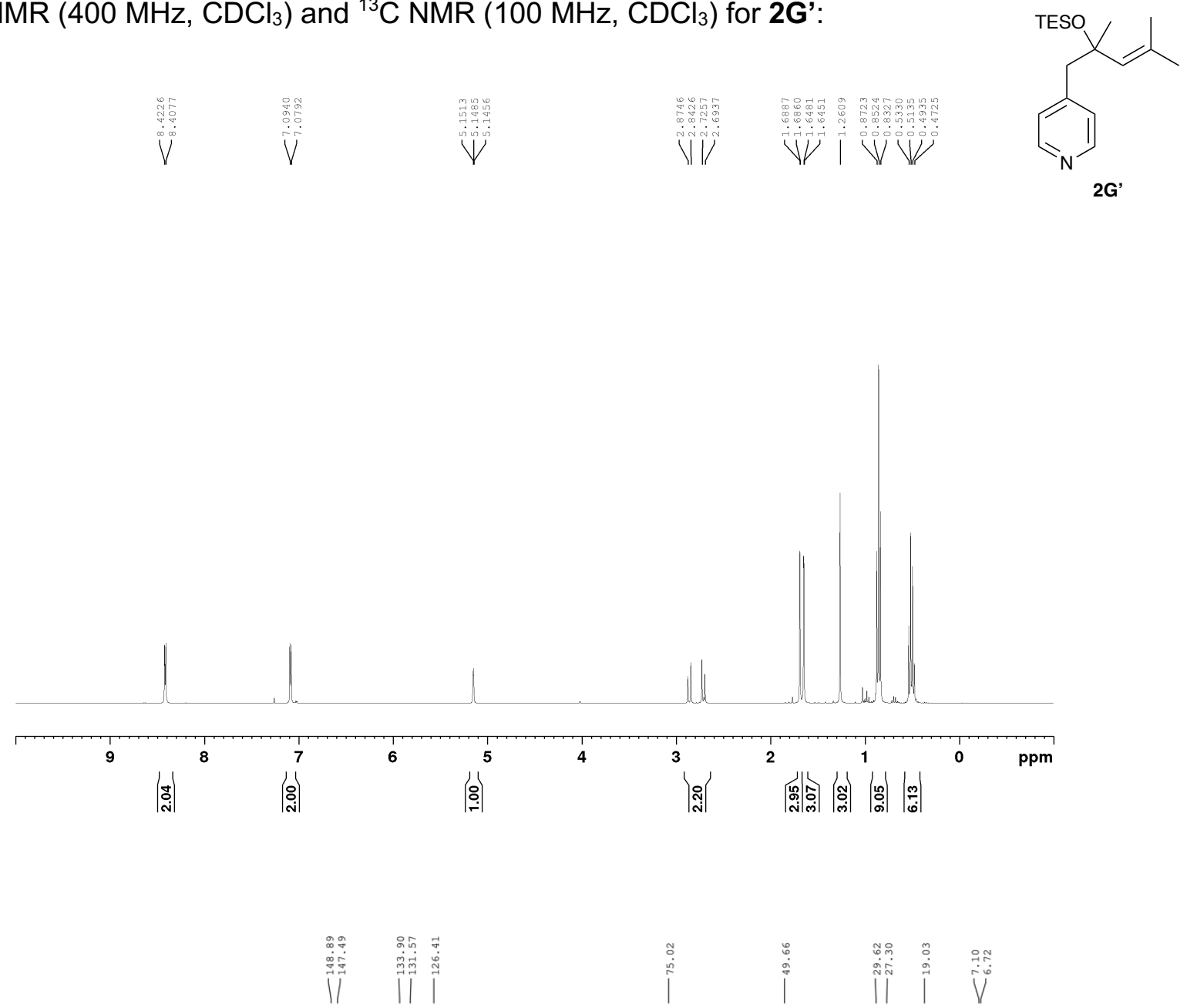

$i^{\stackrel{0}{n}} \quad \stackrel{\circ}{0}$
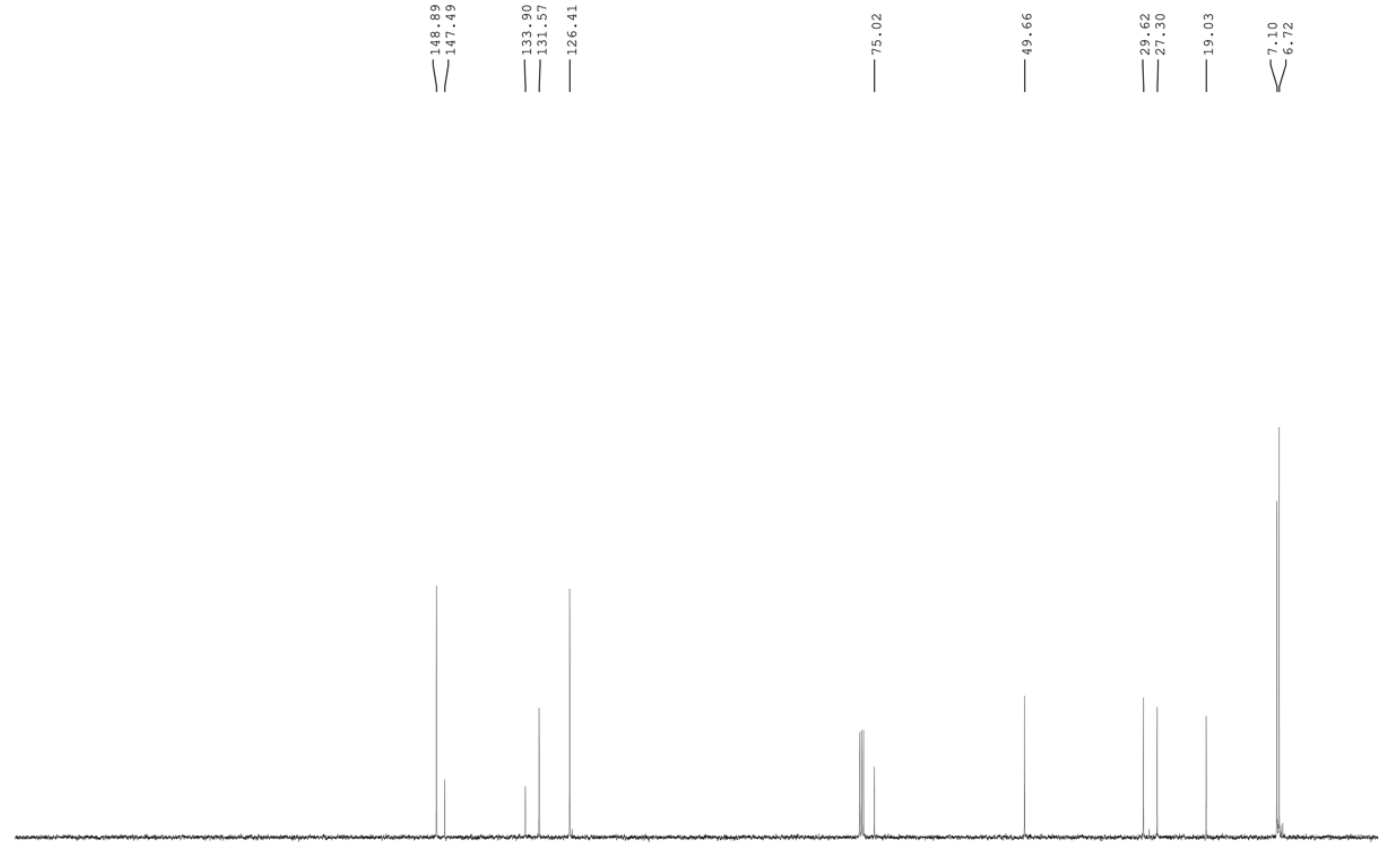

$\begin{array}{lllllllllllllllllllll}210 & 200 & 190 & 180 & 170 & 160 & 150 & 140 & 130 & 120 & 110 & 100 & 90 & 80 & 70 & 60 & 50 & 40 & 30 & 20 & 10\end{array}$ 
${ }^{1} \mathrm{H}$ NMR $\left(400 \mathrm{MHz}, \mathrm{CDCl}_{3}\right)$ and ${ }^{13} \mathrm{C}$ NMR $\left(100 \mathrm{MHz}, \mathrm{CDCl}_{3}\right)$ for 21:
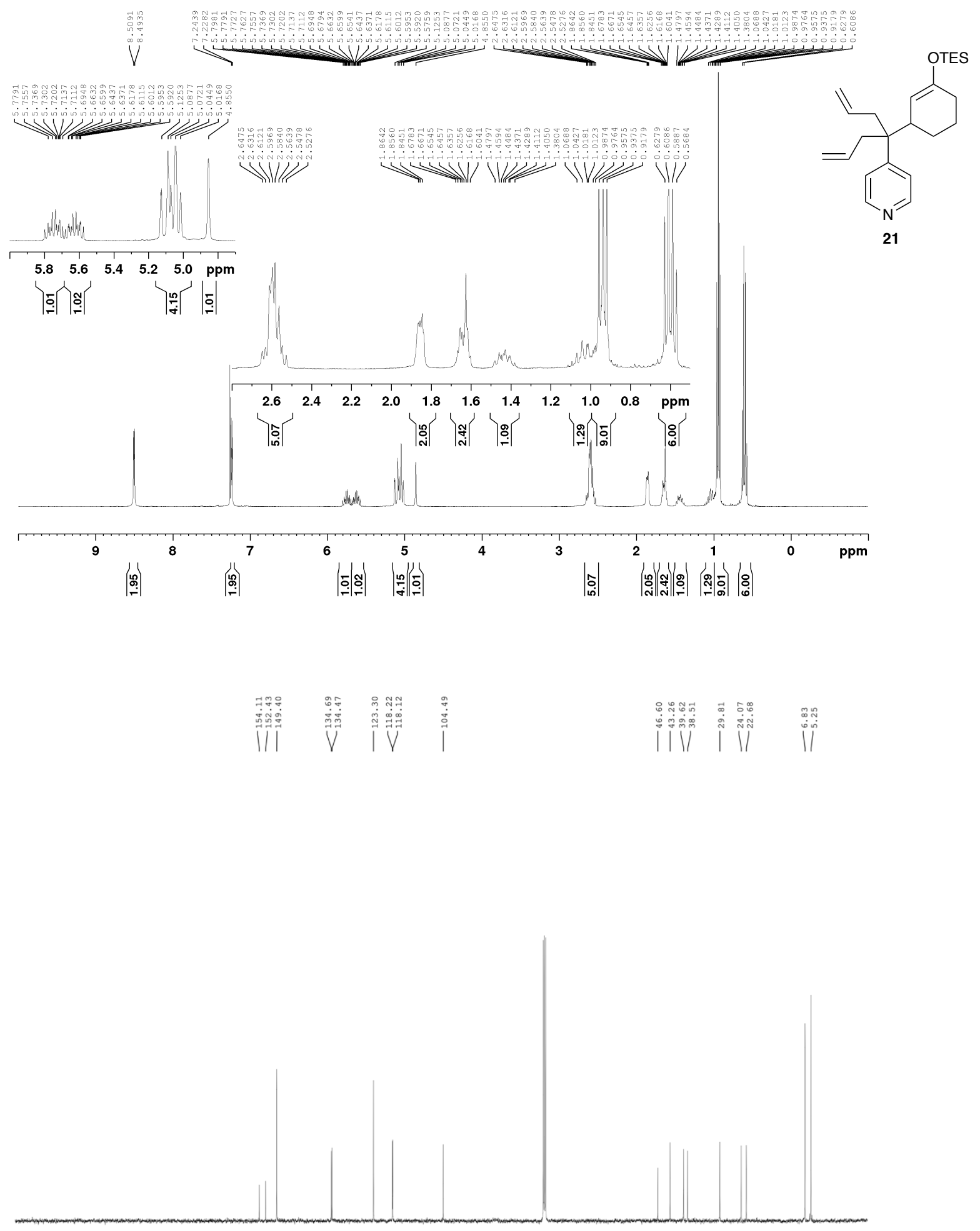

$\begin{array}{llllllllllllllllllllll}210 & 200 & 190 & 180 & 170 & 160 & 150 & 140 & 130 & 120 & 110 & 100 & 90 & 80 & 70 & 60 & 50 & 40 & 30 & 20 & 10 & \mathrm{ppm}\end{array}$ 
${ }^{1} \mathrm{H} \mathrm{NMR}\left(400 \mathrm{MHz}, \mathrm{CDCl}_{3}\right)$ and $\left.{ }^{13} \mathrm{C} \mathrm{NMR} \mathrm{(100} \mathrm{MHz,} \mathrm{CDCl}_{3}\right)$ for 22:
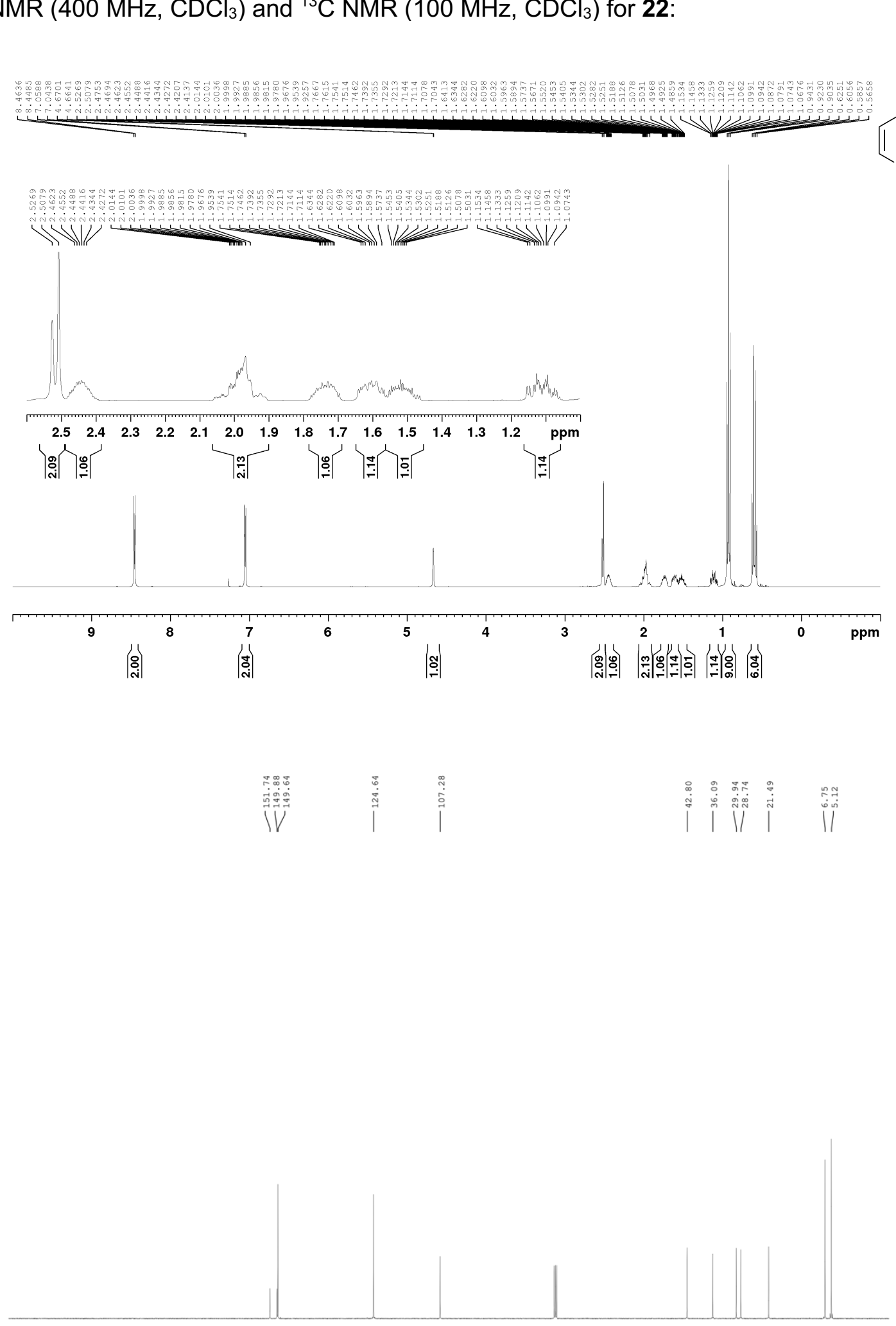

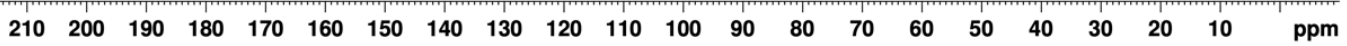


${ }^{1} \mathrm{H} \mathrm{NMR}\left(400 \mathrm{MHz}, \mathrm{CDCl}_{3}\right)$ and $\left.{ }^{13} \mathrm{C} \mathrm{NMR} \mathrm{(100} \mathrm{MHz,} \mathrm{CDCl}_{3}\right)$ for 23:
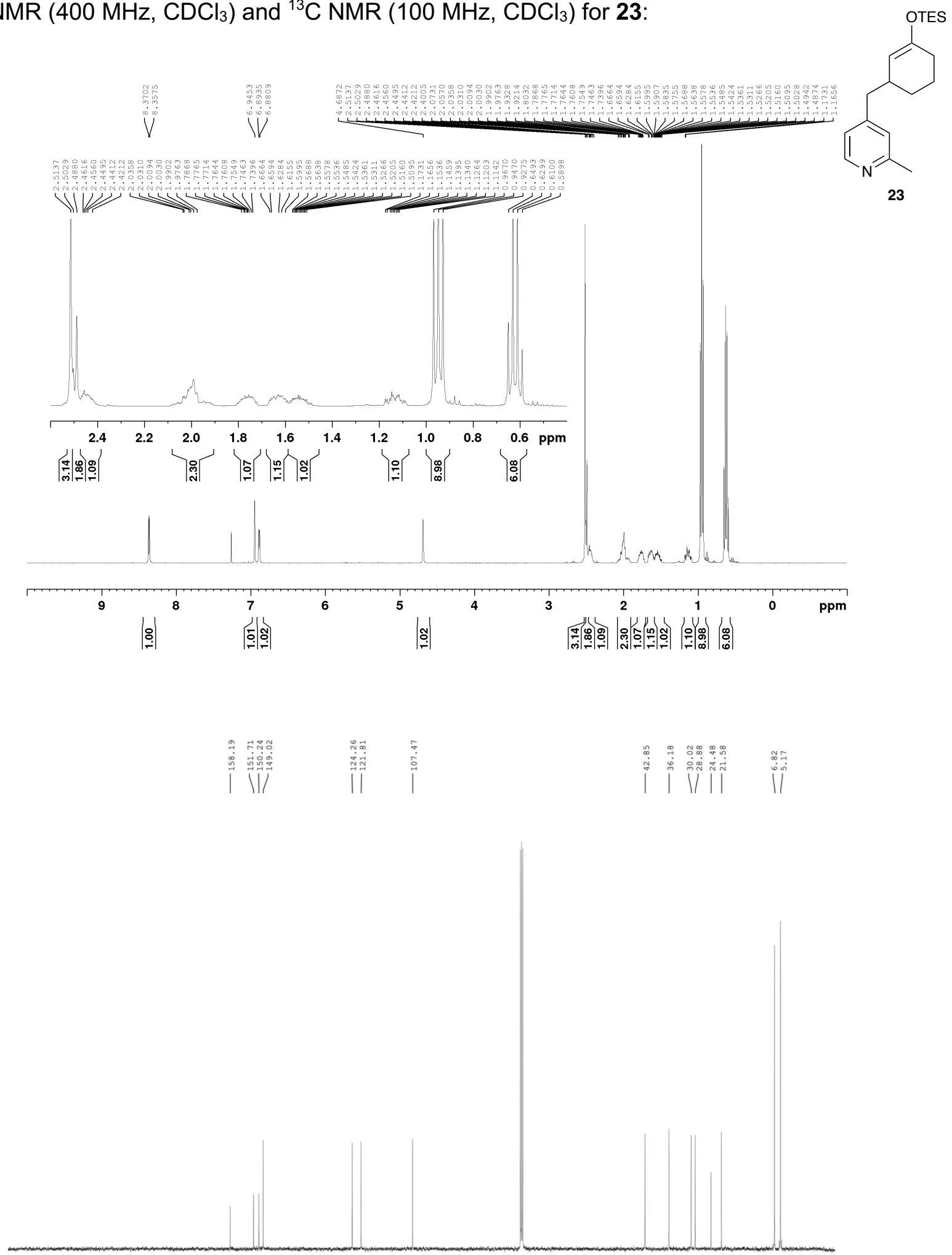

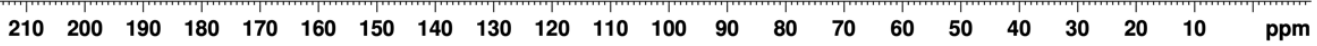

